\title{
Modulation of plasticity aftereffects at the sensorimotor cortex by transcranial electrical and magnetic stimulation
}

\author{
Dissertation \\ for the award of the degree \\ "Doctor of Philosophy" \\ Division of Mathematics and Natural Sciences \\ of the Georg-August-Universität Göttingen
}

within the doctoral program Sensory and Motor Neuroscience

of the Georg-August University School of Science (GAUSS)

submitted by

Mohd Faizal Mohd Zulkifly

from Terengganu, Malaysia

Göttingen 2020 


\section{THESIS COMMITTEE}

Prof. Dr. Walter Paulus (Supervisor)

Department of Clinical Neurophysiology, University Medical Center Göttingen

\section{Prof. Dr. Manuela Schmidt}

Somatosensory Signaling Group, Max Planck Institute for Experimental Medicine, Göttingen.

Prof. Dr. Melanie Wilke

Department of Cognitive Neurology, University Medical Center Göttingen

\section{MEMBERS OF THE EXAMINATION BOARD}

Prof. Dr. Walter Paulus (Referee)

Department of Clinical Neurophysiology, University Medical Center Göttingen

Prof. Dr. Manuela Schmidt ( $2^{\text {nd }}$ Referee $)$

Somatosensory Signaling Group, Max Planck Institute for Experimental Medicine, Göttingen.

Prof. Dr. Melanie Wilke

Department of Cognitive Neurology, University Medical Center Göttingen

\section{FURTHER MEMBERS OF THE EXAMINATION BOARD}

Prof. Dr. Tiago Fleming Outiero

Experimental Neurodegeneration, University Medical Center Göttingen

Prof. Dr. Hansjörg Scherberger

Neurobiology Laboratory, German Primate Center, Göttingen

Prof. Dr. Dr. Hannelore Ehrenreich

Clinical Neuroscience, Max Planck Institute of Experimental Medicine, Göttingen

Date of oral examination: 7th December 2020 


\section{DECLARATION OF AUTHORSHIP}

I hereby declare that the thesis "Modulation of plasticity aftereffects at the sensorimotor cortex by transcranial electrical and magnetic stimulation" has been written independently and with no other sources and aids than quoted.

Mohd Faizal Mohd Zulkifly

Göttingen, $30^{\text {th }}$ October 2020 


\section{ACKNOWLEDGEMENTS}

First and foremost, I would like to thank my supervisor, Prof. Dr. Walter Paulus, for the opportunity given to me to work in this fascinating field of Brain Stimulation. His continues support, guidance and passion in research motivate me to finish this $\mathrm{PhD}$ journey. I also owe my gratitude to other collaborators Prof. Carsten $\mathrm{H}$. Wolters, Institute for Biomagnetism and Biosignalanalysis, University of Muenster and Prof. Jürgen Brockmöller, Department of Clinical Pharmacology, University Medical Center, Georg-August University, Göttingen. Their scientific input and multi-disciplinary collaboration improve the quality of my research work and inspires me to be a good researcher in future.

Furthermore, I want to express my gratitude to Ornela Merkohitaj, a medical doctoral candidate, which also contribute to some part in the research work and also helping me in recruiting the participants. I also want to thank Dr. Albert Lehr, a former colleague and the best companion in my life in Göttingen. His support and kindness when we spent time together as a colleague and endless support until the completion of this dissertation give a meaningful impact on my PhD life.

Also, I want to give a big shout-out to my funding institutions, the Ministry of Higher Education (MoHE), Malaysia and the University of Science Malaysia (USM). A scholarship that was granted to me for four years of my $\mathrm{PhD}$ helped me to continue my studies at the University of Göttingen. This gratitude also goes to the Göttingen Graduate Center for Neurosciences, Biophysics, and Molecular Biosciences (GGNB) of the Georg-August-Universität Göttingen. GGNB also provided me with financial support during the last three months of my PhD studies.

Then, I want to thank Dr Islam Halawa, a former colleague and all of my colleagues in the Department of Clinical Neurophysiology for all the support, the hustle and bustle, every single moments and experience as a team.

And, last but not least, definitely I would like to express my deepest gratitude to my beloved family and my friends for their support, understanding and encouragement over the years of my $\mathrm{PhD}$ journey. Success in life comes from a blessing of our parents and positive people in our surrounding! 


\begin{abstract}
Non-invasive brain stimulation (NIBS) dose-dependently influences ongoing brain activity either by electrical stimulation via electrodes attached to the scalp or by magnetic stimulation via coils. The latter then induces electric currents in the brain. The current state of knowledge suggests that a shift in membrane potential and changes in the neuronal firing rates underlie neuroplastic changes induced by NIBS. Neuroplastic changes lead to structural and functional reorganization that underlies amongst others learning and memory, due to the strengthening or weakening of the synaptic transmission. NIBS methods avoid the risks of invasive implantation associated with deep brain stimulation (DBS). However, when compared with DBS, long-lasting effects (i.e., aftereffects) induced by NIBS are necessary for therapeutic applications. Here response variability and the inconsistent reproducibility of the induced aftereffects remain challenges in the field. Efforts are underway to improve the efficacy of NIBS by optimizing the technical parameters, and others by exploring the intra- and inter-individual factors. In the present thesis, we investigated further confounding factors in plasticity studies at the sensorimotor cortex specifically focusing on modifiable factors. We used both transcranial electrical and magnetic stimulation methods to induce neuroplasticity in healthy human brains. As a readout, we measured the aftereffects using motor evoked potentials (MEPs).
\end{abstract}

The first experiment (chapter 2) explored the effects of caffeine on the plasticity aftereffects of $140 \mathrm{~Hz}$ transcranial alternating current stimulation (tACS) over the motor cortex. We recruited fourteen subjects who do not consume caffeine. Our results showed that the facilitatory aftereffects of tACS were reversed into inhibition after espresso with caffeine and no changes in the plasticity aftereffects after decaffeinated espresso.

Moving forward from the findings in chapter 2, we designed two randomized, placebocontrolled studies (chapter 3 and chapter 4) using a fixed dose of caffeine (200 mg). We measured sixty participants (study 1: caffeine naïve $(n=30)$; study 2: caffeine consumers $(n=30)$ ). Our results in chapter 3 revealed three key findings; 1) caffeine strengthened and prolonged the plasticity aftereffects in caffeine naïve subjects, 2) an increase in alertness during tACS was associated with increases in MEP facilitation, 3) light-deprivation during tACS suppressed the MEP amplitudes in 
caffeine consumers. In chapter 4, we found that higher prestimulation caffeine concentrations were associated with higher baseline cortical excitability in caffeine consumers. In caffeine naïve, higher poststimulation caffeine concentrations were related with lower poststimulation MEPs after Sham. We showed that there were no relationships between poststimulation caffeine, poststimulation corticosteroids concentrations and plasticity aftereffects. Caffeine administration increases the salivary corticosteroid concentrations in both study groups. These corticosteroid concentrations vary significantly over the time of day and was not affected by stimulations.

In summary, caffeine is a major confounding factor, which affects the cortical excitability. Alertness and ambient light joined caffeine as other potential confounders that reduce the efficacy of NIBS and plasticity-induction studies. 
Table of Contents

DECLARATION OF AUTHORSHIP iii

ACKNOWLEDGEMENTS iv

ABSTRACT $\quad$ v

LIST OF TABLES $\quad$ X

LIST OF ILLUSTRATIONS X xi

LIST OF ABBREVIATIONS X xii

CHAPTER I

$1.1 \quad$ General Introduction 1

1.2 Non-invasive Brain Stimulation (NIBS) 2

1.2.1 Transcranial Magnetic Stimulation (TMS) 2

1.2.2 Transcranial Electrical stimulation (tES) 4

$\begin{array}{lll}1.3 & \text { Brain Plasticity } & 6\end{array}$

1.3.1 Mechanisms and Applications 6

1.3.2 Neuroplasticity induced by PAS and tACS in different cortical regions 8

$\begin{array}{lll}1.4 & \text { Response Variability } & 10\end{array}$

1.4.1 Non-modifiable factors and NIBS efficacy 11

1.4.2 Modifiable factors and NIBS efficacy 11

1.5 Outlook and goal of the thesis 13

$\begin{array}{lll}1.6 & \text { Overview of the chapters } & 14\end{array}$

$\begin{array}{lll}\text { 1.6.1 Chapter } 2 & 14\end{array}$

$\begin{array}{lll}\text { 1.6.2 Chapter } 3 & 14\end{array}$

$\begin{array}{lll}1.6 .3 & \text { Chapter } 4 & 15\end{array}$

CHAPTER II 16

$\begin{array}{lll}2.1 & \text { Abstract } & 16\end{array}$

$\begin{array}{lll}2.2 & \text { Introduction \& Results } & 18\end{array}$

$\begin{array}{ll}\text { CHAPTER III } & 23\end{array}$

$\begin{array}{lll}3.1 & \text { Abstract } & 23\end{array}$ 
3.3.1 Participants 28

3.3.2 Transcranial Magnetic Stimulation (TMS) 28

3.3.3 Paired Associative Stimulation (PAS) 29

3.3.4 Transcranial Alternating Current Stimulation (tACS) 29

$\begin{array}{lll}3.3 .5 & \text { Pupillometry } & 30\end{array}$

3.3.6 Study design 31

$\begin{array}{lll}\text { 3.3.7 Data analysis and statistics } & 34\end{array}$

$3.4 \quad$ Results 36

3.4.1 Motor threshold and baseline $\mathrm{MEP}\left(\mathrm{MEP}_{\mathrm{PRE}}\right)$ comparisons 36

3.4.2 Stimulation perception and correct identification of stimulation type 37

3.4.3 Experiment 1: Effects of caffeine on PAS 25 and LTP/LTD-like aftereffects after $140 \mathrm{~Hz}$ tACS in caffeinenaïve participants

3.4.4 Experiment 2: Effects of caffeine, time of day and alertness
during stimulation on cortical excitability in the caffeine

3.4.4 Experiment 2: Effects of caffeine, time of day and alertness
during stimulation on cortical excitability in the caffeine consumer group

3.4.5 Alertness and cortical excitability 44

3.4.6 Caffeine consumption behaviours on motor cortex excitability 47

Discussion

Conclusions

$\begin{array}{lll}\text { 4.3.1 Participants } & 65\end{array}$

4.3.2 Transcranial Magnetic Stimulation (TMS) 66

4.3.3 Paired Associative Stimulation (PAS) 66

4.3.4 Transcranial Alternating Current Stimulation (tACS) 67

$\begin{array}{lll}\text { 4.3.5 Saliva sample collection and analysis } & 67\end{array}$

4.3.6 Experimental design 68

$\begin{array}{lll}\text { 4.3.7 Data analysis and statistics } & 71\end{array}$ 
4.4.1 Salivary caffeine concentrations 72

4.4.2 Salivary cortisone, cortisol and total corticosteroid concentrations 73

4.4.3 Relationship between caffeine and corticosteroids concentration with motor cortex excitability 75

4.4.4 Effects of caffeine, stimulation and time of day on $\begin{array}{ll}\text { circulating corticosteroids } & 78\end{array}$

Discussion

Conclusions

$\begin{array}{lll}5.1 & \text { Summary of the findings } & 91\end{array}$

$\begin{array}{lll}5.2 & \text { Implications and future directions }\end{array}$

$\begin{array}{lll}5.3 & \text { Conclusion } & 96\end{array}$

$\begin{array}{ll}\text { BIBLIOGRAPHY } & 97\end{array}$

$\begin{array}{ll}\text { APPENDIX A } & 117\end{array}$

$\begin{array}{lll}\text { Appendix A.1 CURRICULUM VITAE } & 118\end{array}$ 


\section{LIST OF TABLES}

Table No.

Table $3.1 \quad$ Self-reported alertness scores (PST0 to PST30)

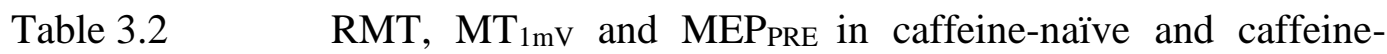
adapted groups

Table 4.

Caffeine concentrations in saliva

Table 4.2

Corticosteroids concentration in saliva

Table 4.3

The correlations of log transformed prestimulation caffeine and prestimulation corticosteroids concentrations with motor thresholds and baseline MEP amplitudes (MEP PRE)

Table 4.4 Experiment 1 (caffeine-naïve): The correlations of $\log$ transformed poststimulation caffeine concentrations with MEP amplitudes at the poststimulation measurement 1 (POST 1), poststimulation measurement 2 (POST 2) and at 30 minutes poststimulation measurement (PST30)

Table 4.5 Experiment 1 (caffeine-naïve): The correlations of $\log$ transformed poststimulation corticosteroid concentrations with MEP amplitudes at the poststimulation measurement 1 (POST 1), poststimulation measurement 2 (POST 2) and at 30 minutes poststimulation measurement (PST30)

Table 4.6 Experiment 2 (caffeine consumers): The correlations of $\log$ transformed poststimulation caffeine concentrations with MEP amplitudes at the poststimulation measurement 1 (POST 1), poststimulation measurement 2 (POST 2) and at 30 minutes poststimulation measurement (PST30)

Table $4.7 \quad$ Experiment 2 (caffeine consumers): The correlations of $\log$ transformed poststimulation corticosteroid concentrations with MEP amplitudes at the poststimulation measurement 1 (POST 1), poststimulation measurement 2 (POST 2) and at 30 minutes poststimulation measurement (PST30) 


\section{LIST OF ILLUSTRATIONS}

Figure No.

Page

Figure 1.1 Illustrations of the induced current in the brain by TMS and tES.

Figure 1.2 Illustration of spike-timing-dependent plasticity (STDP) mechanism.

Figure 2.1 Espresso with or without caffeine was consumed in two different session in randomized order $45 \mathrm{~min}$ before tACS $(n=14)$.

Figure 3.1 Experimental design.

Figure 3.2 Effect of caffeine on plasticity aftereffect following PAS 25 (Experiment 1).

Figure 3.3 Cortical excitability after tACS with or without caffeine (Experiment 1).

Figure 3.4 Cortical excitability with placebo or caffeine (Experiment 2).

Figure 3.5 Pooled data for pupillary oscillations (PUI) and mean pupil diameters during stimulation (Experiment 2).

Figure 3.6 Correlations between pupillary unrest index (PUI) and cortical excitability (MEP amplitudes)

Figure 4.1

Experimental design.

70

Figure 4.2

Salivary concentrations of corticosteroids in all conditions.

80 


\section{LIST OF ABBREVIATIONS}

\begin{tabular}{|c|c|}
\hline A1R & Adenosine Type 1 Receptor \\
\hline $\mathrm{A} 2 \mathrm{R}$ & Adenosine Type 2 Receptor \\
\hline ACTH & Adrenocorticotrophic Hormone \\
\hline BDNF & Brain-Derived Neurotrophic Factor \\
\hline BOLD & Blood-Oxygen-Level-Dependent \\
\hline CSP & Cortical Silent Period \\
\hline DBS & Deep Brain Stimulation \\
\hline DLPFC & Dorsolateral Prefrontal Cortex \\
\hline EEG & Electroencephalography \\
\hline FDA & Food And Drug Administration \\
\hline FDI & First Dorsal Interosseous \\
\hline GABA & Gamma-Aminobutyric Acid \\
\hline HPA & Hypothalamus Pituitary Axis \\
\hline ICF & Intracortical Facilitation \\
\hline LICI & Long-Interval Intracortical Inhibition \\
\hline LTD & Long-Term Depression \\
\hline LTP & Long-Term Potentiation \\
\hline M1 & Primary Motor Cortex \\
\hline MEG & Magnetoencephalography \\
\hline MEP & Motor Evoked Potential \\
\hline MEP PRE $_{1}$ & Baseline MEP Amplitude \\
\hline MN & Median Nerve \\
\hline MRS & Magnetic Resonance Spectroscopy \\
\hline $\mathrm{MT}_{1 \mathrm{mV}}$ & $1 \mathrm{mV}$ peak-to-peak MEPs \\
\hline
\end{tabular}




\begin{tabular}{|c|c|}
\hline NIBS & Non-Invasive Brain Stimulation \\
\hline NMDA & N-Methyl-D-Aspartate \\
\hline NMDAR1 & N-Methyl-D-Aspartate Receptor 1 \\
\hline PAS & Paired Associative Stimulation \\
\hline PD & Pupil Diameter \\
\hline PFC & Prefrontal Cortex \\
\hline PNS & Peripheral Nerve Stimulation \\
\hline PUI & Pupillary Unrest Index \\
\hline QPS & Quadripulse Transcranial Magnetic Stimulation \\
\hline RMT & Resting Motor Threshold \\
\hline RTMS & Repetitive Transcranial Magnetic Stimulation \\
\hline STDP & Spike Timing Dependent Plasticity \\
\hline TACS & Transcranial Alternating Current Stimulation \\
\hline TBS & Theta-Burst Stimulation \\
\hline TEP & Transcranial Evoked Potential \\
\hline TES & Transcranial Electric Stimulation \\
\hline TMS & Transcranial Magnetic Stimulation \\
\hline TPCS & Transcranial Pulsed Current Stimulation \\
\hline TRNS & Transcranial Random Noise Stimulation \\
\hline VSCCS & Voltage-Sensitive $\mathrm{Ca}^{2+}$ Channels \\
\hline
\end{tabular}




\section{CHAPTER I}

\subsection{GENERAL INTRODUCTION}

The success of deep brain stimulation (DBS) in particular in Parkinson's disease encourages noninvasive stimulation approaches as supplement to pharmacological treatment of neuropsychiatric disorders. After that, other techniques for brain stimulation started to emerge with the same translational goal. Transcranial magnetic stimulation (TMS) and transcranial electric stimulation (tES) both are known as non-invasive brain stimulation (NIBS) techniques. TMS produces a magnetic field that penetrates the scalp and the skull that in turn induces an electric field underneath the coil. Suprathreshold intensities change the membrane potentials of neurons, then trigger and synchronize action potentials that affect ongoing brain oscillations. For tES on the other hand, a weak current (typically $\leq 2 \mathrm{~mA}$ ) is applied for $5-20 \mathrm{~min}$ using electrodes on the scalp. The electric field at subthreshold intensity changes the resting membrane potential of neurons to a smaller extent and hereby modulates spontaneous firing rates of action potentials. NIBS-induced changes at the systems level lead to improvement in memory and learning also due to a strengthening or weakening of the synaptic transmission (i.e. long-term potentiation (LTP)-like and long-term depression (LTD)-like plasticity). NIBS is safe, tolerable and potentially useful as adjunctive treatment tool. It's neuroplastic effects (i.e. neurophysiology, behavior) last longer than the stimulation duration and offer options to study intact human brains in research and clinical settings.

After more than 30 years of NIBS research, only TMS (i.e. repetitive transcranial magnetic stimulation) has received full approval from the US Food and Drug Administration (FDA) as a 
treatment for major depression. Therapeutic use of other TMS and tES variants in treating neurological and neuropsychiatric disorders is more experimental at this moment. Strategies are on the way to optimize the aftereffects and reduce variability which include standardizing the methodological or technical aspects of stimulation and also controlling other neurophysiological variables which could confound the neuroplastic changes. Pharmacological agents, brains states, attention are known factors to affect the strength and direction of the plasticity aftereffects. New knowledge of possible confounding factors and their effects on NIBS may help us to overcome the variability concerns and improve the efficacy of non-invasive brain stimulation.

\subsection{NON-INVASIVE BRAIN STIMULATION (NIBS)}

Changes in neurotransmitter releases (e.g., $\gamma$-aminobutyric acid (GABA) and glutamate) play a role in the physiology of NIBS. Glutamate binds to $N$-methyl- $D$-aspartate (NMDA) receptors, and their expression regulates cortical excitability and plasticity in the human cortex (Liebetanz et al., 2002; Mori et al., 2011). NMDA-receptor antagonists (i.e. amantadine) reduce motor cortex excitability after paired-pulse TMS (Reis et al., 2006), whereas NMDA agonists (i.e. $D$ - cycloserine) prolong the MEP facilitation of anodal tDCS substantially by a factor of 20 (Nitsche et al., 2004). By magnetic resonance spectroscopy (MRS) Stagg and colleagues revealed that the facilitatory effects of anodal tDCS are mediated by a reduction in GABAergic inhibition, whereas the inhibitory effects of cathodal tDCS are mediated by a reduction in glutamatergic neurotransmission (Stagg et al., 2009). On the membrane level, $\mathrm{Ca}^{2+}$ channels are involved. The facilitatory effects of paired associative stimulation (PAS) and theta-burst stimulation (TBS) was blocked with an L-type voltage gated $\mathrm{Ca}^{2+}$ channels blocker (i.e. nimodipine) (Wankerl et al., 2010; Weise et al., 2017).

\subsubsection{Transcranial Magnetic Stimulation (TMS)}

TMS is a non-invasive method that generates a magnetic field which induces electric currents in the brain (Figure 1.1a). There are several ways for applying TMS which include single-pulse TMS (i.e. one stimulus at a time), paired-pulse TMS (i.e. pairs of stimuli separated by a variable interval) and repetitive TMS (i.e. trains of stimuli). Single-pulse TMS is used to map the motor cortex and 
record the outputs. Paired-pulse TMS (e.g. paired associative stimulation - PAS) can be used to measure intracortical facilitation and inhibition, and also investigate cortico-cortical interactions. Repetitive TMS - i.e. conventional TMS (repetitive TMS - rTMS), patterned TMS (e.g. theta burst stimulation - TBS; quadripulse stimulation - QPS) are protocols used to induce plastic changes in the brain which outlast the stimulation period (Rossi et al., 2009). TMS is capable of inducing plastic changes in the brain either strengthening (LTP) or weakening (LTD) the synapses depending on the stimulation protocols, and it has a useful clinical application (Hallett, 2007; Müller-Dahlhaus, Ziemann and Classen, 2010).

Stimulation frequency, intensity and duration are important parameters for guiding rTMSinduced aftereffects. Trains of stimuli applied at low-frequency $(<1 \mathrm{~Hz})$ lead to inhibitory effects, whereas with high-frequency stimulation $(>5 \mathrm{~Hz}$ ) produces excitatory effects (Paulus, Peterchev and Ridding, 2013; Klomjai, Katz and Lackmy-Vallée, 2015). Higher frequency rTMS is limited to intensities below the motor threshold because of the risk of inducing seizures (Rossi et al., 2009). A new paradigm of rTMS has been developed with shorter stimulation times, lower intensities which make it, most importantly, more tolerable. Paired associative stimulation (PAS) combines repetitive time-locked pairings of TMS over the primary motor cortex (M1) with peripheral nerve stimulation (PNS). For example, the peripheral stimulation is applied at median nerve and its effects recorded as changes in the MEP amplitudes (Stefan et al., 2000). Plasticity aftereffects depend on the inter-stimulus interval (ISI) between TMS and nerve stimulation. An interval of $20-25 \mathrm{~ms}$ (PAS 25) resulted in LTP-like effects, while shorter ISI of around $10 \mathrm{~ms}$ (PAS 10) led to LTD-like aftereffects (Wolters et al., 2003; Weise et al., 2013).

PAS follows the concept of Hebbian synaptic plasticity (Hebb, 1949). A recent study showed that not only spike-timing but also firing rate are both responsible for inducing plasticity in PAS. In their protocol, higher TMS frequency $(0.2 \mathrm{~Hz})$ than the conventional frequency $(0.05$ $\mathrm{Hz})$ with a higher frequency of PNS $(100 \mathrm{~Hz})$ is the most effective in inducing MEP facilitation (Tolmacheva, Mäkelä and Shulga, 2019). In a review by Suppa and colleagues (Suppa et al., 2017), the authors addressed that PAS has beneficial effects in neurological and psychiatric disorders. There is higher MEPs facilitation in chronic stroke after PAS. Besides that, more severe patients will receive more benefits from the stimulation (Silverstein et al., 2019). An interesting study in 
cerebral ischemic rats demonstrated the molecular and functional changes related to plasticity inductions after PAS. Molecular changes induced by PAS was shown by an increase in the level of brain-derived neurotrophic factor (BDNF) and $N$-methyl- $D$-aspartate receptor 1 (NMDAR1) proteins expression and thus improved learning and memory in the cerebral ischemic rats (Hu et al., 2019).

\subsubsection{Transcranial Electrical Stimulation (tES)}

In 1980, a method to induce an electrical current through scalp electrodes in the intact brain was developed by (Merton and Morton, 1980). It was a breakthrough as the technique can induce muscle twitches after short-lasting and strong electrical impulses (i.e. $2000 \mathrm{~V}$ in $10 \mu \mathrm{s}$ ) applied over the motor cortex. However, the technique was painful and intolerable. Twenty years later, weak transcranial direct current stimulation technique was invented by (Nitsche and Paulus, 2000). The stimulation at subthreshold current intensity is a reliable method in modulating cortical excitability. It also alters the spontaneous activity of neurons (Nitsche and Paulus, 2000, 2011), which sustains the aftereffects of up to $40 \%$ and lasted for between 30 and 120 minutes depending on the stimulation montages (Kuo et al., 2013). Over the years, other tES techniques were developed, which include transcranial alternating current stimulation (tACS), transcranial random noise stimulation (tRNS), and transcranial pulsed current stimulation (tPCS). These low-intensity stimulations (intensity: < $4 \mathrm{~mA}$, stimulation duration: up to $60 \mathrm{~min}$ ) is considered safe and tolerable with considerable adverse events were reported (Bikson et al., 2016; Antal et al., 2017).

The computational and in vitro studies showed that soma and axon terminals are responsible for modulation of the neuronal excitability during tDCS. The shift in the transmembrane potential depends on neuronal orientation (Rahman et al., 2013; Rahman, Lafon and Bikson, 2015). Also, in vivo studies demonstrated that modulation of the NMDA receptors, changes in intracellular $\mathrm{Ca}^{2+}$, alteration in neurotransmitters and neuromodulators release are responsible for inducing neuroplastic changes (Liebetanz et al., 2002; Nitsche et al., 2004, 2012; Stagg, Antal and Nitsche, 2018). Recently, more research started to investigate the modulatory effects of tACS on different domains of brain functions and uncover its underlying mechanisms. Unlike tDCS, tACS current is sinusoidal, which means that the polarity between anodal to cathodal 
is reversed periodically. (Figure 1.1b). tACS works by modulating the neuronal membrane potential and also entraining the neuronal oscillations (Zaehle, Rach and Herrmann, 2010; Paulus, 2011; Antal and Paulus, 2013; Herrmann et al., 2013; Reato et al., 2013; Antal and Herrmann, 2016; Tavakoli and Yun, 2017). Thus, it has the unique potential to affect different domains of brain functions and to treat neurological disorders with abnormal brain rhythms. tACS is a parameter-sensitive stimulation technique and its effects were shown both during and after stimulation depending on stimulation protocols (Moliadze, Antal and Paulus, 2010; Herrmann et al., 2013; Vossen, Gross and Thut, 2015; Abellaneda-Pérez et al., 2020). Low intensity and short stimulation duration do not affect changes in the MEPs, EEG amplitude or phase (Antal et al., 2008; Strüber et al., 2015). Apart from that, the aftereffects are frequency-specific; for instance, $20 \mathrm{~Hz}$ tACS ( $B$ range) on the motor cortex increases the MEPs. In contrast, the stimulation at other frequency bands (i.e. $5 \mathrm{~Hz}, 10 \mathrm{~Hz}$ and $40 \mathrm{~Hz}$ ) do not change the MEPs (Feurra et al., 2011). Similarly, only tACS at $140 \mathrm{~Hz}$ increased motor cortex excitability when tACS was applied at ripples frequencies $(80,140,250 \mathrm{~Hz}$ ) (Moliadze, Antal and Paulus, 2010). Working memory improvements were reported in different tACS montages such as targeting fronto-parietal circuits (Polanía et al., 2012) and prefrontal regions (Alekseichuk et al., 2016). Consistent behavioural and electrophysiological aftereffects of tACS were recently shown by (Kasten and Herrmann, 2017). They showed that an increase in ongoing alpha power and coherence during mental rotation tasks resulted in an increase of the performance both during and after tACS. 
a)

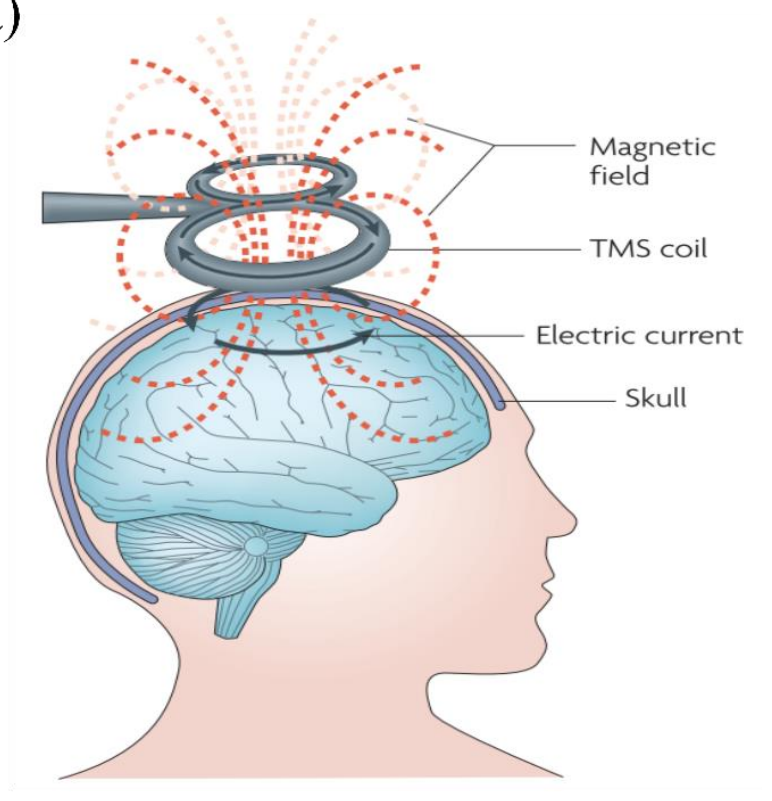

b)

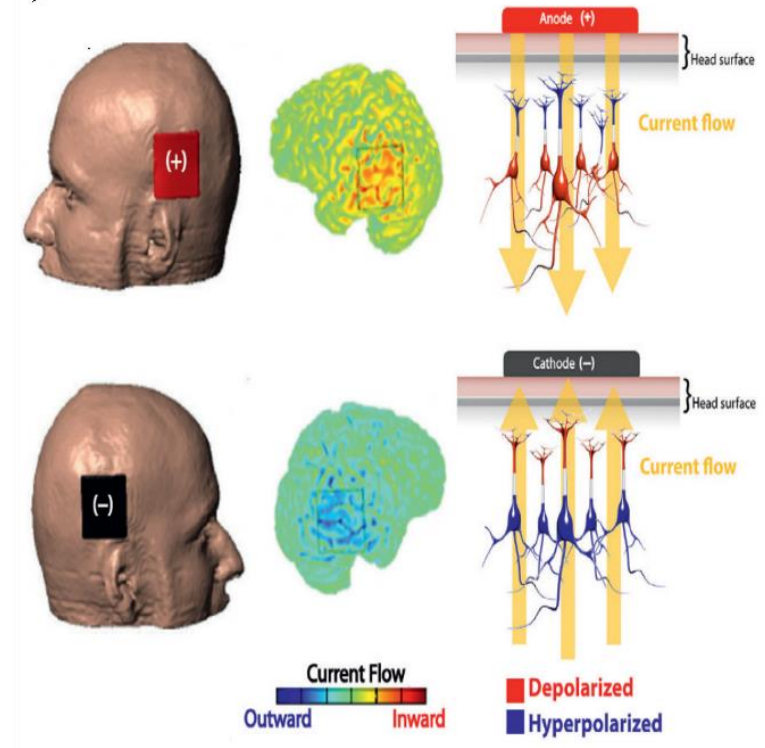

Figure 1.1 Illustrations of the induced current in the brain by TMS and tES.

a) The magnetic field produced by the TMS coil induces electric currents in the brain. A suprathreshold current depolarizes the neurons and generates the action potential. Adapted from (Ridding and Rothwell, 2007). b) A low-intensity current or at sub-threshold of tES changes the membrane potential and thus affects neuronal excitability. Anodal stimulation generates an inward current flow, depolarizes the soma of pyramidal neurons and leads to an excitability increase under the electrode. In contrast to cathodal stimulation, an outward current hyperpolarizes the soma of pyramidal neurons and decreases the cortical excitability. For tACS/tRNS, the current flow is fluctuating in time and then the membranes alternate between depolarization and hyperpolarization. Adapted from (Moreno-Duarte et al., 2014).

\subsection{BRAIN PLASTICITY}

\subsubsection{Mechanisms and Applications}

Stefan and colleagues introduced PAS protocols to investigate the Hebbian principle of synaptic plasticity in humans (Stefan et al., 2000). In this protocol, median nerve (MN) stimulation was paired with TMS repetitively with a certain interstimulus interval (ISI) to produce robust and long-lasting changes in the stimulated cortex for up to $60 \mathrm{~min}$ (Stefan et al., 2000, 2002; Wolters et al., 2005; Müller-Dahlhaus, Ziemann and Classen, 2010; Carson and Kennedy, 2013; MüllerDahlhaus et al., 2015). PAS-induced LTP/LTD-like plasticity is a synapse-specific protocol (Nitsche et al., 2007), and the direction of cortical excitability changes depends on timing rules. 
PAS aftereffects follow the Hebbian plasticity principle and resemble the spike timing dependent plasticity (STDP) (Wolters et al., 2003, 2005; Müller-Dahlhaus, Ziemann and Classen, 2010). The relative timing between pre- and post-synaptic action potential determines the direction of plasticity inductions, inhibition or excitation. LTP-like plasticity is triggered after repetitively the firing of the postsynaptic potential is followed by the presynaptic potential. In turn, if postsynaptic neurons fire before presynaptic firing, it then leads to LTD (Lisman and Spruston, 2010). (See Figure 1.2a). a). A series of pharmacological studies revealed that PAS LTP/LDP-like plasticity is dependent on NMDA receptor activation and voltage-dependent $\mathrm{Ca}^{2+}$ channels (Stefan et al., 2002; Wolters et al., 2003; Wankerl et al., 2010). Interestingly, PAS induced cortical excitability changes are not only observed at the stimulation site (i.e. sensorimotor regions), but also in the excitability of cortical circuits. TMS-evoked cortical EEG responses, spectral power changes (i.e. alpha and beta) showed that the induced aftereffects of PAS also extended contralaterally to the stimulation site (Huber et al., 2008; Naro et al., 2014). The interstimulus interval (ISI) between median nerve (MN) pulse and the TMS pulse modulates the MEP amplitude (Wolters et al., 2003) and the P25 amplitude (Wolters et al., 2005). In a comparative review from different TMS protocols, PAS has a comparable effect size to conventional rTMS and generally longer aftereffects duration than conventional rTMS (Thut and Pascual-Leone, 2010).

It is a constant debate if either entrainment of brain oscillations or spike-timing-dependent plasticity (STDP) is responsible for the observed aftereffects of tACS. Like PAS, neuroplastic effects of tACS follow a similar mechanism in terms of the effects on the spiking activity of stimulated neurons at a certain temporal window. Consistent evidence from simulation and humans studies support that plasticity mechanisms underlie the induced aftereffects (Zaehle, Rach and Herrmann, 2010; Strüber et al., 2015; Vossen, Gross and Thut, 2015; Wischnewski and Schutter, 2017). For instances, Vossen and colleagues exclude the entrainment echoes as a contribution to aftereffects in their intermittent tACS paradigm by showing the absence of phase-locking immediately after tACS ended (Vossen, Gross and Thut, 2015). A well-known STDP mechanism using alpha-tACS as a model to explain plasticity aftereffects was described by (Zaehle, Rach and Herrmann, 2010). In this model, if the exogenous frequency (i.e. $10 \mathrm{~Hz}$-tACS) was applied at the endogenous resonance frequency, then the repetitive firing during the stimulation strengthened the synapses. This synaptic changes outlasted the stimulation and enhanced the neural activity at the 
resonance frequency (i.e. increase alpha power after $10 \mathrm{~Hz}$-tACS). (See Figure 1.2b). b). The neuroplasticity effects of tACS were later confirmed by a pharmacological study which reported that the effect of beta tACS was abolished in the presence of a NMDA receptor antagonist (i.e. dextromethorphan) (Wischnewski et al., 2019). This indicates that blocking effects at the pre- and post-synaptic neurons change the plasticity induced by tACS.

a)

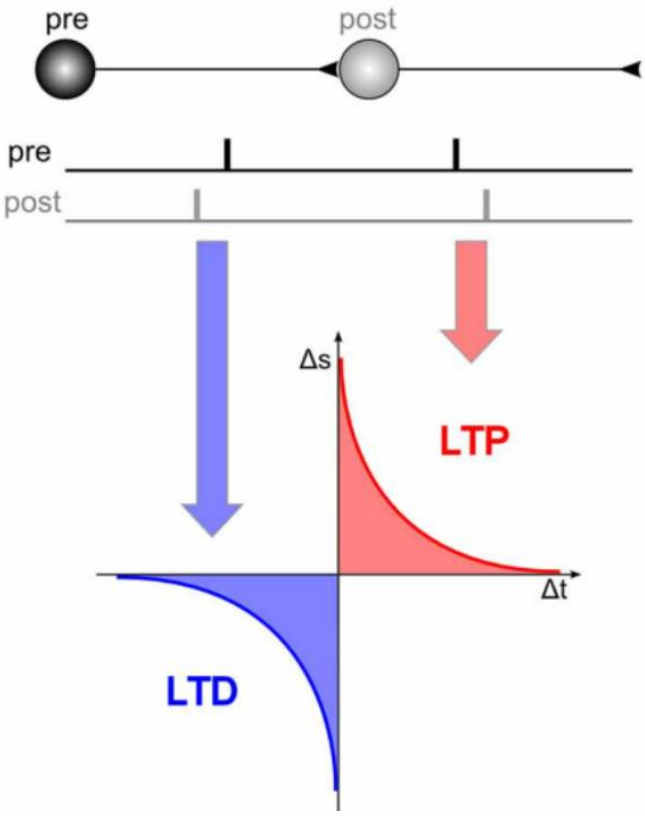

b)

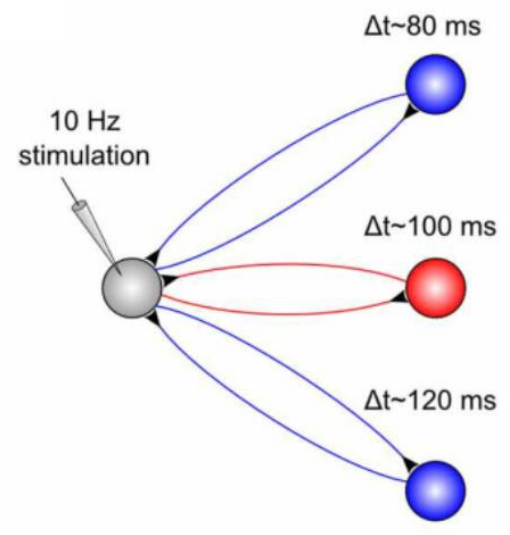

Figure 1.2 Illustration of spike-timing-dependent plasticity (STDP) mechanism.

a) Synapses are strengthened if post- synaptic potential follows a pre-synaptic spike (LTP, in red), whereas synapses are weakened if post-synaptic potential occurs before a pre-synaptic spike (LTD, in blue). b) LTP-like plasticity follows if the driving neurons was stimulated at $10 \mathrm{~Hz}$ (in red) and LTD-like plasticity if the neurons fire earlier or later than a frequency of the driving neurons (in blue). Adapted from (Herrmann et al., 2013).

\subsubsection{Neuroplasticity induced by PAS and tACS in different cortical regions}

The plasticity aftereffects of both PAS and tACS usually are measured at the motor cortex in the form of MEPs (Stefan et al., 2000; Moliadze, Antal and Paulus, 2010; Moliadze et al., 2012; Müller-Dahlhaus et al., 2015; Kortuem et al., 2019), blood-oxygen-level-dependent (BOLD) signal (i.e. reduction in the task-related BOLD response after alpha tACS; Vosskuhl et al. 2016), 
SEPs and spectral changes from EEG/MEG (Wolters et al., 2005; Huber et al., 2008; Zaehle, Rach and Herrmann, 2010; Neuling, Rach and Herrmann, 2013; Ambrus et al., 2015; Vossen, Gross and Thut, 2015; Kasten, Dowsett and Herrmann, 2016) and improvement in behavioural outcomes (Jung and Ziemann, 2009; Ambrus et al., 2015; Kasten and Herrmann, 2017). LTP/LTD-like plasticity effects of PAS are widely studied in the sensorimotor cortex and indexed by changes in the MEPs and SEPs amplitude. In addition to the MEP facilitation as an outread, there was a longlasting reduction of alpha and beta power after PAS (Naro et al., 2014). The authors also showed a relationship between changes in spectral power and MEP excitability increase in the motor cortex (Naro et al., 2014). Most importantly, PAS provides valuable information in terms of an interaction between brain stimulation and learning effects. Jung and Ziemann showed that a LTD- like plasticity protocol increases motor learning (Jung and Ziemann, 2009). This finding corroborates that synaptic plasticity is responsible for the learning effects.

A few studies showed an increase in alpha power which can last up to 30 minutes after posterior alpha tACS (Zaehle, Rach and Herrmann, 2010; Neuling, Rach and Herrmann, 2013; Vossen, Gross and Thut, 2015). In contrast to the somatosensory cortex, there was a reduction in mu-alpha oscillations after tACS applied at participant's individual mu-alpha frequency (Gundlach et al., 2017). Recently, a decrease in alpha power in the somatosensory region after tACS was confirmed by Gundlach and colleagues, which indicates a decrease in functional connectivity between S1 and somatosensory networks (Gundlach et al., 2020). Changes in the power of alpha activity can be explained as a top-down process, in which the functions in the task-irrelevant regions were inhibited (i.e. increase alpha power) and the excitability in the task-relevant regions increased (Diepen, Foxe and Mazaheri, 2019). The alpha inhibition hypothesis follows the evidence from previous studies that neural activity is negatively correlated with alpha-band amplitude (Ploner et al., 2006; Haegens, Händel and Jensen, 2011). Interestingly, the aftereffect of alpha tACS $(10 \mathrm{~Hz})$ is not limited to changes in brain oscillation at the stimulated frequency but affects other frequency bands as well. For instance, during isometric contraction, there was a reduction in cortico-muscular coherence amplitude in the low gamma band after $10 \mathrm{~Hz}$ tACS on the motor cortex (Wach et al., 2013). In the sensorimotor domain, the first clinical benefits of tACS (20 min at $15 \mathrm{~Hz}, 1.5 \mathrm{~mA}$ ) was shown to improve dystonic symptoms up to 30 days with a bipolar C3- C4 montage (Angelakis et al., 2013). Excitability in the motor cortex increased after 
$5 \mathrm{~Hz}$ tACS and $20 \mathrm{~Hz}$ tACS, indicating that $\theta$ and $\beta$ frequency range contribute to motor cortex excitability (Schutter and Hortensius, 2011). Long-lasting MEPs facilitation up to 30-60 min was reported after tACS was applied in the $\mathrm{kHz}$ range $(1,2$ and $5 \mathrm{kHz})$. This finding further supports that membrane excitability mediates the neuroplastic changes and does not interfere with brain oscillations (Chaieb, Antal and Paulus, 2011). Moliadze and colleagues took one step further to apply tACS at the ripple frequency and found robust MEP facilitation after $140 \mathrm{~Hz}$ tACS at 1.0 $\mathrm{mA}$ on the motor cortex. Interestingly, there is a non-linear relationship between current intensity with induced aftereffects. The authors showed inhibitory effects at the stimulation intensity of 0.4 $\mathrm{mA}$, no MEPs changes at the $0.6 \mathrm{~mA}$ and $0.8 \mathrm{~mA}$ intensity and an excitability increase at $1.0 \mathrm{~mA}$ (Moliadze, Antal and Paulus, 2010; Moliadze et al., 2012). Changes in intracellular calcium are thought to determine the magnitude and direction of plasticity as mediated by the current intensities (Stagg, Antal and Nitsche, 2018).

\subsection{RESPONSE VARIABILITY}

The plasticity aftereffects of synaptic strengthening or weakening, which lasts beyond the time of stimulation, offer a great opportunity as a therapeutic tool. However, aftereffects induced by NIBS protocols are not straight forward as intra- and inter-individual variability are a big threat to the field. This might destroy the hopes of NIBS community to use these techniques as therapeutic tools in treating neuropsychiatric disorders. Minkova and colleagues reported that only $61 \%$ of their study population showed the PAS-LTP like effects (Minkova et al., 2019) and only 52\% of the study population in a previous report responded in an expected way (Müller-Dahlhaus et al., 2008). The numbers of responders are slightly lower in another study in which $39 \%, 45 \%$ and $43 \%$ responded to PAS 25, atDCS, and iTBS respectively (López-Alonso et al., 2014). Uncovering the factors which lead to large response variability is a key to optimize the effectiveness and increase potential as a therapeutic approach in the future (Ridding and Ziemann, 2010; Ziemann and Siebner, 2015). In general, we can divide the source of variability into external and internal factors. External factors may include technical and statistical factors such as coil orientation (Talelli et al., 2007; de Goede, ter Braack and van Putten, 2018), current directions (Tremblay et al., 2017; Rawji et al., 2018; Hannah, Iacovou and Rothwell, 2019), stimulation protocols (LópezAlonso et al., 2014; Corp et al., 2020) and sample size (Goldsworthy et al., 2014). Meanwhile, 
internal factors can be classified into three main categories which are neuroanatomical (e.g. cortical thickness; (Conde et al. 2012), neurochemical and neurophysiological determinants (for a review, refer Karabanov et al. 2016). All of them can be either modifiable or non-modifiable factors; the former factors offer a great opportunity to improve the efficacy of the NIBS.

\subsubsection{Non-modifiable factors and NIBS efficacy}

Individual brain anatomy, functional and structural connectivity, age, gender and genetic polymorphisms are known non-modifiable factors which might influence NIBS-induced plasticity (Müller-Dahlhaus et al., 2008; Ridding and Ziemann, 2010; Minkova et al., 2019). In addition, genetic variants such as BDNF gene and its polymorphism are also determinants for NIBS responses. BDNF expression modulates NMDA receptors, a known mechanism which mediates synaptic plasticity (for a review, refer (Chaieb et al., 2014; Saghazadeh, Esfahani and Rezaei, 2016)). Previous studies demonstrated that ' $V a l$ ' allele carriers are more responsive to TBS (i.e. cTBS and iTBS) and rTMS than 'Met' allele carriers (Cheeran et al., 2008; Antal et al., 2010). In contrast, 'Met' allele showed a larger excitability increase after anodal tDCS (Antal et al., 2010). Müller-Dahlhaus and colleagues demonstrated that younger subjects showed larger PAS induced LTP/LTD-like plasticity compared to those who are older (Müller-Dahlhaus et al., 2008). In a recent multicenter study, smaller baseline MEPs were associated with high TBS effectiveness (Corp et al., 2020). The authors also addressed a measurement at the first dorsal interosseous (FDI) muscle which produced a greater iTBS- induced facilitatory effect than the abductor pollicis brevis (APB) muscle.

\subsubsection{Modifiable factors and NIBS efficacy}

Most of the literature focuses currently on modifiable factors as the sources of variability to NIBSinduced plasticity. Priming effects, target muscle states, brain states, time of day, pharmacological agents, circadian rhythms, alertness fluctuation, neurotransmitter availability and hormones levels are known factors in this category (Zarkowski et al., 2006; Sale, Ridding and Nordstrom, 2007; Silvanto, Muggleton and Walsh, 2008; Ridding and Ziemann, 2010; Goldsworthy et al., 2014; Kuhn et al., 2016; Ogata et al., 2019; Noreika et al., 2020). A few series of CNS active drugs 
studies showed that active drugs are robustly modulating the magnitude and direction of cortical plasticity (for a review, refer Nitsche et al. 2012; Ziemann et al. 2015). For instance, NMDA receptor targeting drugs (e.g. memantine, dextromethorphan and $d$-cycloserine) modulate the magnitude and direction of plasticity aftereffects of TBS, PAS and tDCS (Liebetanz et al., 2002; Stefan et al., 2002; Huang et al., 2007). Caffeine is an adenosine receptor antagonist that was recently shown as one of the pharmacological agents which reduced the LTP-like effects induced by quadripulse magnetic stimulation (QPS; Hanajima et al. 2019). In addition, neurophysiological responses such as fluctuations in alertness over the course of the experiment is a source of intertrial variability in MEP and TEP amplitude (Zarkowski et al., 2006; Noreika et al., 2020). Stefan and colleagues addressed that directing participant's attention to the stimulated hand enhanced the plasticity aftereffects in the motor cortex induced by PAS (Stefan, Wycislo and Classen, 2004). The cognitive state and the activity level of the muscle during the stimulation change the effectiveness of tDCS. In the earlier study, Antal and colleagues showed that the direction of tDCSinduced plasticity in the motor cortex changes if the subject performs cognitive tasks during stimulation (Antal et al., 2007). In a later study, the authors showed that there is an increase in the excitability during reading tasks and tDCS effects are affected by reading (Antal, Ambrus and Chaieb, 2014). Other studies demonstrated that MEPs increased during and after 5 Hz-rTMS when attention is directed to the stimulated hand but there was no observable change of MEPs after 1 Hz-rTMS (Conte et al., 2007, 2008). This was attributed to premotor-to-motor connections as it involved the fronto-parietal network which is associated with the 'self-recognition' process (Conte et al., 2007). This indicates that involvement of other cortical areas related to motor cortex influences the excitability in the motor cortex. Pre-activation of the stimulated cortex also reduces the LTD-like plasticity induced by cTBS. Thus, Goldsworthy and colleagues recommend to control the baseline of the target muscle activity to reduce source of variability (Goldsworthy et al., 2014).

A reduction in the phosphene threshold and increase in BOLD signals indicated an increase in visual cortex excitability after light deprivation (Boroojerdi et al., 2000). The aftereffects of alpha power increase or decrease after tACS depend on the ambient light. Darkness further increases the alpha activity, thus reducing the modulatory effects of alpha tACS due to ceiling effects (Stecher et al., 2017). Circadian rhythms including hormone levels and the sleep regulation 
cycle are other factors which need to be considered in plasticity induction studies. Salivary cortisol changes over the day, being lower in the afternoon than in the morning (Mezzullo et al., 2016). Sale and colleagues showed high reproducibility of PAS in the afternoon when cortisol concentration is low and recommends to carry out PAS experiments at a fixed time, preferably in the afternoon, to reduce the variability (Sale, Ridding and Nordstrom, 2007). A reduction in the salivary cortisol concentration after anodal tDCS on the prefrontal cortex (PFC) compared to sham stimulation indicates that this technique modulates neuroendocrine systems (Brunoni et al., 2013; Antal et al., 2014). Hypothalamus pituitary axis (HPA)-system sensitivity was attenuated by leftsided high frequency (HF)-rTMS applied to the left dorsolateral prefrontal cortex (DLPFC) which resulted in decreases in salivary cortisol (Baeken et al., 2014). Besides cortisol, salivary cortisone, a metabolite of salivary cortisol is newly used as a stress biomarker and was linked to autonomic stress markers (i.e. state-trait anxiety and heart rate) (Bae et al., 2019). In addition, there is an increase in cortical excitability in the motor cortex after sleep deprivation as shown by lower TMS intensity and higher EEG theta power compared with sleep condition (Kuhn et al., 2016). Increased theta activity during wakefulness is an indicator of synaptic potentiation (Tononi and Cirelli, 2006). Kuhn and colleagues also showed that the LTP-like plasticity induced by PAS was diminished after sleep deprivation. Also, sessions with sleep deprivation impaired the performance in a declarative memory task compared to sleep condition. A decrease in the LTP-like plasticity after sleep deprivation in their study was associated with a decrease in BDNF plasma level. This might be a protective mechanism to reduce excessive excitability and avoid impairment in the information processing (Kuhn et al., 2016).

\subsection{OUTLOOK AND GOAL OF THE THESIS}

After more than 30 years the reproducibility concerns are one of the major challenges to the field of non-invasive brain stimulation. Some of the confounding factors which lead to variability in healthy subjects were extensively studied. Among them are age, sex, attention, pharmacological agents, genetics, diurnal rhythms and brain states (Ridding and Ziemann, 2010). There are other unknown factors which potentially influence the plasticity aftereffects and thus contribute to nonreproducibility in plasticity studies. In the next chapters (i.e. chapter 2, 3 and 4), I discuss a series of studies on a few potential internal factors which are modifiable and controllable for optimizing 
the efficacy of the brain stimulation techniques. Here we recruited caffeine-naïve and caffeineadapted subjects to comparatively examine their responses to the brain stimulation. In this thesis, MEP was used as the readout of plasticity aftereffects in the motor cortex.

\subsection{OVERVIEW OF THE CHAPTERS}

\subsubsection{Chapter 2}

Caffeine is a psychostimulant which is commonly consumed on a day-to-day basis. It is an adenosine receptor antagonist which is known to arouse, increase cognitive performance and muscle strength. At the synaptic level, it was shown to interfere with GABAergic neurotransmission and regulate intracellular calcium levels. Caffeine is potentially one of the internal factors which might confound plasticity studies. In this chapter, we explored the effects of espresso containing caffeine on the plasticity aftereffects on the motor cortex. We recruited caffeine naïve participants to exclude a potential history of caffeine intake affecting the result. We hypothesized that caffeine enhances the aftereffects and it boosts up the effects of plasticity inducing protocol.

\subsubsection{Chapter 3}

In this chapter, we combined two studies from different populations, caffeine naïve and caffeine consumers. As a continuation of chapter 2, we properly designed both studies to be randomized controlled double-blinded trials and fixed the dose of caffeine to $200 \mathrm{mg}$ in a tablet form. Here, we addressed three important internal factors which may influence the outcome of neuroplasticity studies, which include caffeine intake, time of plasticity induction and alertness during stimulation. We wished to show different plasticity effects of caffeine-naïve and caffeine-adapted subjects. We hypothesized that a higher dose of caffeine $(200 \mathrm{mg})$ than in chapter 2 increases the cortical excitability further and that diurnal rhythms affect plasticity aftereffects. In this study, we also wanted to prove the membrane resistance and shunting inhibition hypothesis by using alertness as a confounding factor affecting the plasticity aftereffects (Paulus and Rothwell, 2016) 


\subsubsection{Chapter 4}

NIBS was reported to affect the neuroendocrine system by affecting the circulating stress hormone cortisol (Antal et al., 2014; Cirillo et al., 2017). This chapter contains the outcomes from two studies which were conducted in chapter 3 . Here, we specifically explored the relationships between salivary caffeine, corticosteroid concentrations and cortical excitability and also plasticity aftereffects. In addition, we also addressed the effects of corticosteroids as another confounding factors on the neuroplasticity aftereffects. We hypothesized that an increase in salivary caffeine and corticosteroids concentrations inhibit the plasticity aftereffects. Further, we hypothesized that caffeine affects the corticosteroid concentrations and stimulations induced changes in the corticosteroid concentrations. 


\section{CHAPTER II}

TRANSCRANIAL ALTERNATING CURRENT STIMULATION INDUCED EXCITATORY AFTEREFFECTS ARE ABOLISHED BY DECAFFEINATED ESPRESSO AND REVERSED INTO INHIBITION BY ESPRESSO WITH CAFFEINE

Published as:

Zulkifly MFM, Merkohitaj O, Paulus W. 2020. Transcranial alternating current stimulation induced excitatory aftereffects are abolished by decaffeinated espresso and reversed into inhibition by espresso with caffeine. Clin Neurophysiol. 131:778-779.

\subsection{ABSTRACT}

Caffeine is a widely used psychostimulant, which acts as a competitive inhibitor to adenosine receptors. Here we investigated whether uncontrolled caffeine intake could interfere with the response to sinusoidal transcranial alternating current stimulation (tACS) with $1 \mathrm{~mA}$ and $140 \mathrm{~Hz}$ applied over the primary motor cortex. We administered espresso, since it reflects everyday situations somewhat better than caffeine tablets. Here, we recruited 14 caffeine non-consumers and they were given espresso with (Nescafe Dolce Gusto®, Espresso Intenso) or without (Nescafe Dolce Gusto®, Espresso Intenso) caffeine on two separate sessions in a randomized order and separated by five days. The participants were blinded to the type of espresso. Forty-five minutes after drinking the espresso, an excitatory tACS protocol $(140 \mathrm{~Hz}, 1 \mathrm{~mA}, 10 \mathrm{~min})$ was applied. In 
order to avoid residual effects of the espresso components we recruited another 14 caffeine nonconsumers as a separate control group treated in a manner identical to the espresso group but without espresso intake. The effects of caffeine are normally associated with cognitive enhancement. However, we did not observe a further enhancement of MEPs after stimulation but rather the opposite; i.e. a decrease in amplitude to $22.1 \%$ below baseline after consuming espresso with caffeine. In addition, MEP amplitudes after decaffeinated espresso consumption were intermediate, as if the increase in excitability after tACS had been simply cancelled out and not reversed. We therefore recommend that both caffeine-containing and decaffeinated beverages should be avoided prior to tACS studies in order to ensure conditions for optimal data reproducibility. 


\subsection{INTRODUCTION \& RESULTS}

Caffeine is a widely used psychostimulant. Here we investigated whether uncontrolled caffeine intake could interfere with the long-term potentiation (LTP)-like response to sinusoidal transcranial alternating current stimulation (tACS) with $1 \mathrm{~mA}$ and $140 \mathrm{~Hz}$ applied over the primary motor cortex. We administered espresso, since it reflects everyday situations somewhat better than caffeine tablets. Espresso also contains other substances in addition to caffeine, which can also modulate brain functions, such as cognition.

A recent study using quadripulse transcranial magnetic stimulation (QPS) has already shown that LTP-like effects are reduced two hours after administration of a $200 \mathrm{mg}$ caffeine tablet (Hanajima et al., 2019). The authors speculated that caffeine might reduce cortical plasticity via a competitive antagonistic effect at the A1 (A1R) and A2A adenosine receptors located both on neurons and glial cells. Caffeine competes with adenosine for receptor binding and has a higher affinity to synaptic A1R than adenosine. Binding at these receptors promotes excitation of synaptic transmission, thereby increasing the excitability of pyramidal neurons in the human neocortex. It is well known that administration of the A1 adenosine receptor antagonist DCPCX reduces the long-term depression (LTD)-like effect of cathodal transcranial direct current stimulation (Marquez-Ruiz et al., 2012). Chronic caffeine consumption induces tolerance, which is associated with an increased number of A1R binding sites in the brain. In addition to cognition, many other functions of the brain can be affected, e.g. those involved in emotional states. Invasive recordings using deep brain stimulation electrodes implanted to treat tremor showed that binding to A1R suppressed excitatory transmission in the thalamus and thus reduced both tremor and deep brain stimulation-induced side effects (Bekar et al., 2008). The effect of adenosine was attenuated by the administration of DPCPX (Marquez-Ruiz et al., 2012). An adenosine receptor antagonist such as caffeine could thus attenuate the effectiveness of DBS in the treatment of tremor and other movement disorders. 
With local ethics committee approval, we recruited 14 caffeine non-consumers (eight female; mean age \pm SD: $23.43 \pm 3.07$ yrs; range: $20-31$ yrs). They were given espresso with (Nescafe Dolce Gusto ${ }^{\circledR}$, Espresso Intenso, caffeine condition) or without caffeine (Nescafe Dolce Gusto ${ }^{\circledR}$, Espresso Intenso, decaf condition) on two separate sessions in a randomized order and separated by five days. The participants were blinded to the type of espresso. Forty-five minutes after drinking the espresso, an excitatory tACS protocol $(140 \mathrm{~Hz}, 1 \mathrm{~mA}, 10 \mathrm{~min})$ was applied over the primary motor cortex (Moliadze, Antal and Paulus, 2010). In order to avoid residual effects of the espresso components we recruited another 14 caffeine non-consumers as a separate control group treated in a manner identical to the espresso group but without espresso intake (eight female; mean age \pm SD : $23.5 \pm 2.2$ yrs; range: $20-28$ yrs). Single-pulse TMS was used to record motor evoked potentials (MEPs) of the right first dorsal interosseous muscle (FDI) with surface $\mathrm{Ag}-\mathrm{AgCl}$ electrodes in a belly-tendon montage. MEPs were induced using a Magstim 2002 magnetic stimulator (Magstim Co. Ltd., Whitland, Wales, UK) with the D70 coil. This coil was held tangentially to the skull, with the handle pointing backwards and laterally at $45^{\circ}$ from the midline. The electromyography signals were amplified, band-pass filtered $(2 \mathrm{~Hz}-2 \mathrm{kHz})$, and digitized at a sampling rate of $5 \mathrm{kHz}$ with a micro $1401 \mathrm{AD}$ converter (Cambridge Electronic Design Ltd., Cambridge, UK). All signals were stored on a computer for offline analysis and the peak-to-peak MEP amplitudes were read with a customized script. The subjects were asked to relax during MEPs recording. We recorded 25 trials of baseline MEPs. Immediately after tACS we recorded MEP responses every five minutes for 30 minutes.

Statistical analysis was performed using SPSS software (IBM SPSS statistics 26; IBM Corp., Armonk, NY, USA). The six measurements post-tACS were averaged to one post-tACS data point (POST). A repeated measures (rm) ANOVA was implemented with CONDITION3 levels x TIME2levels.. The p-value for statistical significance was $<0.05$. Fisher's LSD test was performed if a significant main effect of CONDITION and the interaction of CONDITION $x$ TIME were observed. Shapiro-Wilk-Test was used to test the distribution of the data and all of them were normally distributed.

The average baseline MEP amplitude for control, decaf and caffeine conditions were (mean $\pm \mathrm{SD}$ ) $=1.03 \pm 0.08 \mathrm{mV}, 1.06 \pm 0.13 \mathrm{mV}, 1.04 \pm 0.11 \mathrm{mV}$ respectively (Figure 1). We reproduced 
the previously demonstrated LTP-like effect of the tACS (Moliadze, Antal and Paulus, 2010) by showing a 26.2\% increase of MEP amplitude above baseline (Figure 1). RmANOVA showed a significant main effect of CONDITION $(\mathrm{F}(2,26)=4.23, \mathrm{p}=0.026)$, and a significant interaction effect of CONDITION X TIME $(F(2,26)=6.26, p=0.006)$. Post hoc tests showed a significant decrease in MEP amplitude after tACS in the caffeine condition compared to the control group (Fisher's LSD test, $\mathrm{p}<0.05$ ). Paired t-test showed that tACS in the control group increased MEP amplitude compared with baseline $(\mathrm{t}(13)=-2.57, \mathrm{p}=0.023)$, while espresso with caffeine reversed the tACS-induced MEP increase into inhibition $(\mathrm{t}(13)=2.65, \mathrm{p}=0.02)$.

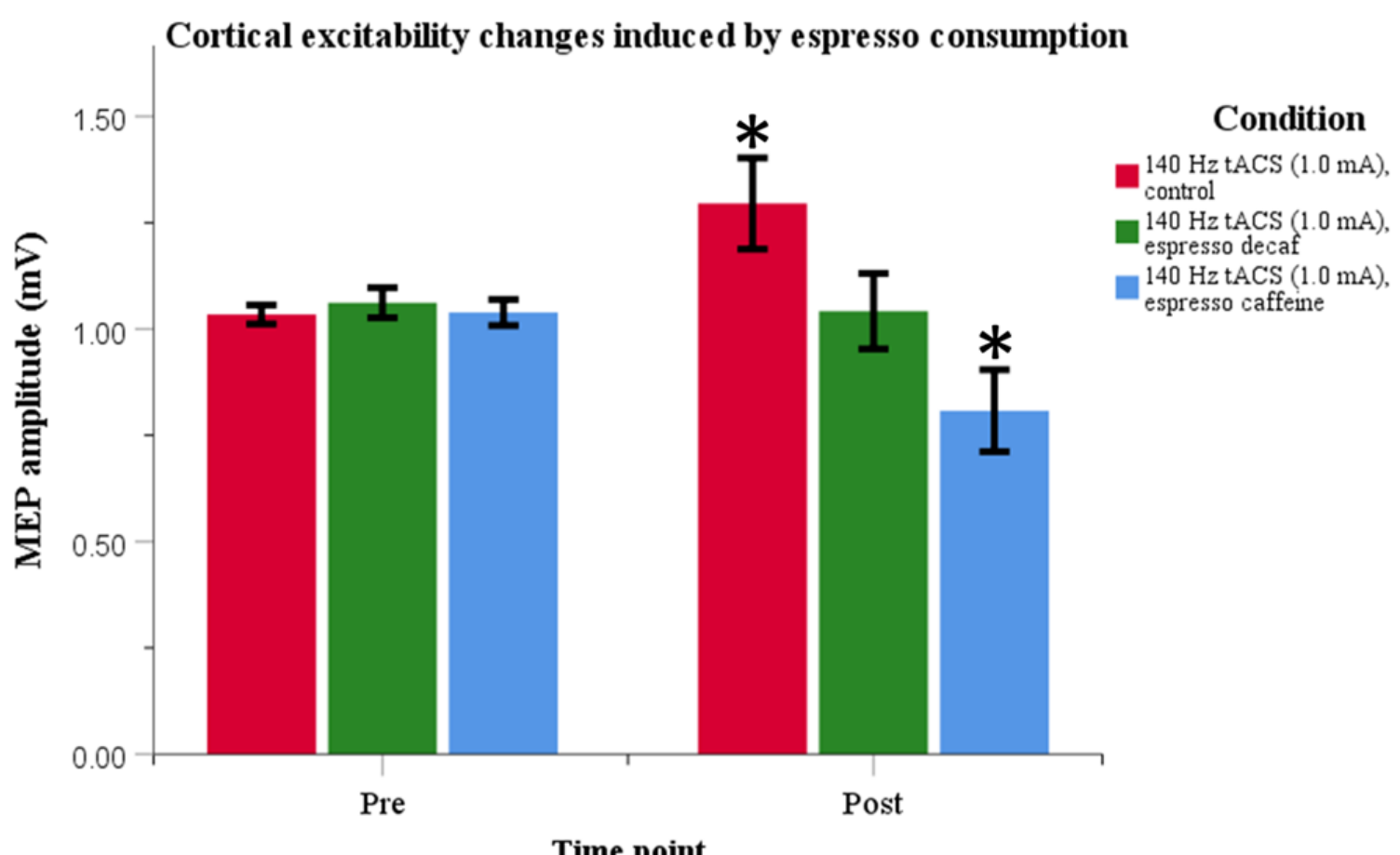

Figure 2.1 Espresso with or without caffeine was consumed in two different session in randomized order 45 min before tACS $(\mathrm{n}=14)$.

No espresso was consumed in a control group of different subjects $(n=14)$. MEPs increased significantly over baseline in the control group (paired t-test, $\mathrm{p}=0.023$ ), decreased significantly after espresso with caffeine (paired t-test, $\mathrm{p}=0.02$ ), and did not change from baseline after decaffeinated espresso. Data are shown as means \pm SEM and $*$ indicates $p<0.05$.

Abbreviations: $\mathrm{MEP}=$ motor evoked potential; $\mathrm{tACS}=$ transcranial alternating current stimulation. 
The effects of caffeine are normally associated with cognitive enhancement. However, we did not observe a further enhancement of the tACS-induced LTP-like effect but rather the opposite; i.e., a significant LTD-like effect to $22.1 \%$ below baseline after consuming espresso with caffeine. This was, at first sight, unexpected, since both $140 \mathrm{~Hz}$ tACS with $1 \mathrm{~mA}$, as well as caffeine are known to increase intracellular $\mathrm{Ca} 2+$ concentrations. $\mathrm{Ca} 2+$ at a saturation level inactivates the ion channels that ultimately change the direction of plasticity. In animal brain slices, accumulation of intracellular $\mathrm{Ca} 2+$ beyond a certain level blocked the voltage-sensitive $\mathrm{Ca} 2+$ channels (VSCCs) and impeded e.g. LTP induced by theta-burst stimulation (Yasuda, Sabatini and Svoboda, 2003). In addition, caffeine also modulates neuronal membrane resistance by inducing an inward current, which could affect GABAergic inhibitory transmission, e.g. by increasing intracellular calcium. These observations support the view that LTP-plasticity depends critically on the exact level of the intracellular $\mathrm{Ca} 2+$ concentration.

After tACS in the decaffeinated espresso condition, MEP amplitude did not change compared to baseline, i.e., an intermediate effect between the control and caffeine conditions (Figure 1). An explanation for this might be that other bioactive compounds remaining after decaffeinating the roasted coffee beans interfered with the LTP-like effect of tACS. Among these are chlorogenic acids (CGAs), trigonelline, tryptophan alkaloids, and diterpenes.

We therefore recommend that both caffeine-containing and decaffeinated beverages should be avoided prior to tACS, and possibly other non-invasive brain stimulation techniques, in brain plasticity induction studies in normal physiology and clinical settings, in order to ensure conditions for optimal data reproducibility.

\section{Funding}

MFMZ was supported by the Ministry of Education (MOE), Malaysia.

\section{Conflict of Interest}

The authors declare no conflict of interest. 


\section{Acknowledgements}

The authors thank Prof. Thomas Crozier for proofreading and Dr. Islam Halawa for his contributions to the experimental procedures. 


\section{CHAPTER III}

CONFOUNDING EFFECTS OF CAFFEINE ON NEUROPLASTIC EFFECTS INDUCED BY TRANSCRANIAL ALTERNATING CURRENT STIMULATION (TACS) AND 25MS PAIRED ASSOCIATIVE STIMULATION (PAS25)

Submitted as:

Mohd Faizal Mohd Zulkifly, Ornela Merkohitaj, Jürgen Brockmöller, Walter Paulus. Confounding effects of caffeine on neuroplastic effects induced by transcranial alternating current stimulation (tACS) and 25ms paired associative stimulation (PAS25).

\subsection{ABSTRACT}

Objective: We examined the effects of caffeine, time of day, and alertness fluctuation on plasticity effects after transcranial alternating current stimulation (tACS) or 25ms paired associative stimulation (PAS25) in caffeine-naïve and caffeine-adapted subjects. Methods: In two randomised, double-blinded, cross-over or placebo-controlled (caffeine) studies, we measured sixty subjects in eight sessions ( $n=30$, Male: Female $=1: 1$ in each study). Results: We found caffeine increased motor cortex excitability in caffeine naïve subjects. The aftereffects in caffeine naïve subjects were enhanced and prolonged when combined with PAS 25. Caffeine also increased alertness and the MEPs were reduced under light deprivation in caffeine consumers both with and without caffeine. In caffeine consumers, the time of day had no effect on tACS-induced plasticity. Conclusions: We conclude that caffeine should be avoided or controlled as confounding factor for brain stimulation protocols. It is also important to keep the brightness constant in all sessions and study groups should not be mixed with caffeine-naïve and caffeine consuming participants. Significance: Caffeine is one of the confounding factors in the plasticity induction studies and it induces different excitability effects in caffeine-naïve and caffeine-adapted subjects. 
This study was registered in the ClinicalTrials.gov with these registration IDs:

1) NCT03720665

https:/clinicaltrials.gov/ct2/results? cond=NCT03720665\&term $=\&$ cntry $=\&$ state $=\&$ city $=\&$ dist $=$

\section{2) NCT04011670}

https://clinicaltrials.gov/ct2/results? cond=\&term $=$ NCT04011670\&cntry $=\&$ state $=\&$ city $=\&$ dist $=$

Keywords: alertness; cortical excitability; motor cortex; plasticity; transcranial magnetic stimulation (TMS) 


\subsection{INTRODUCTION}

Multiple mechanisms contribute to synaptic plasticity, which plays a key role in learning, memory and many other brain processes. Spike timing-dependent plasticity (STDP) is a widely accepted theory that addresses the importance of timing between pre- and postsynaptic spikes as a determinant that strengthens or weakens synaptic transmission (Markram, 2011; Mateos-Aparicio and Rodríguez-Moreno, 2019). Non-invasive transcranial brain stimulation (NIBS) techniques, specifically transcranial magnetic stimulation (TMS) of the motor cortex, allow one to quantify synaptic plasticity in the intact human brain (Pötter-Nerger et al., 2009; Karabanov et al., 2015; Huang et al., 2017; Polanía, Nitsche and Ruff, 2018). Furthermore, NIBS can modulate plasticity through various mechanisms. Transcranial alternating current stimulation (tACS) at specific frequencies modulates the excitability of the motor cortex (Zaehle, Rach and Herrmann, 2010; Vossen, Gross and Thut, 2015). Repetitive pairing of TMS pulses with an afferent input (Paired Associative Stimulation (PAS)) induces synapse-specific plastic aftereffects by modulating Nmethyl-D-aspartate (NMDA) receptors and voltage-dependent calcium channels (Carson and Kennedy, 2013; Suppa et al., 2017). Due to the multitude of influencing factors, such as individual differences, protocol design, and the neuronal states of the stimulated areas (Ridding and Ziemann, 2010; Huang et al., 2017) reproducibility issues need to be resolved or at least clarified (Goldsworthy et al., 2014; López-Alonso et al., 2014; Ziemann and Siebner, 2015; Hordacre et al., 2017; Huang et al., 2017; Guerra et al., 2020).

Caffeine is a widely used psychostimulant. It is consumed by approximately $85 \%$ of the US population with an average intake of $165 \mathrm{mg} /$ day in the form of coffee and caffeinated beverages (Mejia and Ramirez-Mares, 2014; Mitchell et al., 2014). Caffeine is a competitive adenosine receptor antagonist with a greater affinity to the adenosine type 1 receptor (A1R) than to other types of adenosine receptors. In the brain, caffeine not only inhibits the A1R, it also interferes with GABAergic synapses by suppressing inhibitory GABAergic neurotransmission, regulates intracellular potassium and calcium levels, and at the systems level shortens the cortical silent period (CSP) (Cerqueira et al., 2006; Ferreira et al., 2014; Isokawa, 2016; Kerkhofs et al., 
2018; Calker et al., 2019). On the other hand, the well-known arousal effect of caffeine is mediated by activation of the adenosine type 2 receptor (A2R), since A2R knockout mice do not exhibit the arousal effects of caffeine (Huang et al., 2005). Other effects target memory, attention, and performance, increase muscle strength (Duncan and Oxford, 2011; Mesquita et al., 2020), regulate the sleep-wake cycle (Landolt, 2008), influence emotional states (Calker et al., 2019), and modulate visual perception (Nguyen et al., 2018).

During deep brain stimulation, A1Rs are activated by the release of adenosine, which then inhibits excitatory transmission in the thalamus and thereby reduces tremor (Bekar et al., 2008). This effect of reducing tremor was attenuated in the presence of the adenosine A1 receptor antagonist DPCPX by restoring the increase in excitatory transmission (Bekar et al., 2008). An accumulation of adenosine and an increase in binding activity to the adenosine A1 receptor inhibit the adenylate cyclase-cAMP-protein kinase A signaling pathway (Calker, Müller and Hamprecht, 1979), thereby decreasing protein kinase $C$ and calcium levels which in turn result in a decrease in excitatory neurotransmission (Islam et al., 1994, 1995). In cathodal transcranial direct current stimulation, administration of DCPCX prevents the long term depression (LTD)-like effects (ctDCS) (Marquez-Ruiz et al., 2012). Excitatory theta-burst stimulation (iTBS) in rats showed a significantly reduced long-term potentiation (LTP)-like effect in a caffeine-treated group (Blaise et al., 2018). The authors speculated that chronic consumption of caffeine for three weeks caused an up-regulation of A1R as a compensatory response. There are mixed outcomes of caffeineinfluenced plasticity effects in man. The LTP-like effects of quadripulse transcranial magnetic stimulation (QPS) are reduced after the administration of a $200 \mathrm{mg}$ caffeine tablet (Hanajima $e t$ al., 2019). In caffeine-naïve participants, caffeine reversed the LTP-like aftereffects of $140 \mathrm{~Hz}$ tACS into inhibition, whereas in the group without caffeine (Zulkifly, Merkohitaj and Paulus, 2020) the excitatory tACS aftereffects were simply cancelled out, resulting in no effect. A study using anodal transcranial direct current stimulation (atDCS) demonstrated that stimulation increased muscle strength, but a combination of stimulation with caffeine did not augment the effects on muscle strength (Lattari et al., 2019). Smaller amounts of caffeine such as those found in energy drinks reduced intracortical facilitation (ICF) and improved motor performance as shown by stronger MEP facilitation and shorter reaction times in the PAS 25 paradigm after a motor task (Concerto, Infortuna, et al., 2017). There are no systematic dosage studies of the effects of caffeine 
with stimulation protocols, which does not allow one to draw a general conclusion about a dosedependent response at this moment.

Caffeine, as in coffee, influences the level of attention, which in turn modulates plastic aftereffects. Besides caffeine, brain states during stimulation are widely reported to influence stimulation-induced plasticity. Compared to a resting condition, plasticity in the motor cortex (M1) was decreased in participants when stimulation with anodal tDCS occurred while performing an unspecific cognitive task (Antal et al., 2007). Vice versa, in PAS the plastic aftereffects were enhanced when attention was focused on targeted external stimuli (Stefan, Wycislo and Classen, 2004) or when attention was focussed on a target muscle that received a vibratory stimulus (Rosenkranz and Rothwell, 2006). The plastic aftereffects of tDCS changed significantly in a reading task during stimulation as shown by a lower phosphene threshold (Antal, Ambrus and Chaieb, 2014). Changes in brain states such as fatigue level, wakefulness, preconditioning, eye opened/closed and light deprivation all modulate neuronal responses and thus change plasticity effects (Boroojerdi et al., 2000; Kraemer et al., 2000; Leon-Sarmiento, Bara-Jimenez and Wassermann, 2005; Silvanto, Muggleton and Walsh, 2008; de Graaf et al., 2017; Chen and Huang, 2018). Different brain states in the stimulated and connected areas can therefore mask the dynamic of interactions within and between neurons and lead to a suboptimal intervention. Internal factors such as time of plasticity induction also modulate cortical plasticity. The effectiveness of PAS in inducing plasticity aftereffects was determined by time of day as it was reported that the MEP facilitation was greater in the evening than in the morning sessions (Sale, Ridding and Nordstrom, 2007, 2008).

In bringing all this evidence together, three important factors may influence the outcome of neuroplasticity studies, which include caffeine intake, time of plasticity induction and alertness during stimulation as a brain state factor. Acute caffeine intake affects the caffeine-naïve subjects and chronic caffeine users in different ways and modulates a synapse-specific (PAS) and an unspecific (tACS) stimulation protocol differently. We hypothesized that cortical excitability would be increased after caffeine administration and that tACS-induced plasticity would be more effective in the afternoon. In addition, in view of evidence that increased attention during stimulation modulates plasticity aftereffects, we wished to verify alertness as one of the 
components of attention that is correlated with changes in cortical excitability. In particular, we pursued the hypothesis that attention via activating cell ensembles goes along with opening of more membrane channels. This leads to an increase in membrane conductance or loss of membrane resistance which in turn will weaken transcranial stimulation effects via leaky membranes (Paulus and Rothwell, 2016). To achieve these aims, we studied the effects of $200 \mathrm{mg}$ caffeine on PAS 25 and tACS in LTP/LTD-like plasticity protocols. We also explored the influence of alertness during stimulation on cortical excitability and examined its relationship.

\subsection{MATERIAL AND METHODS}

\subsubsection{Participants}

We recruited our participants from students of the University of Göttingen. They were screened for right-handedness, caffeine consumption, a history of neurological or psychiatric disorders, and any contraindication to brain stimulation. An ECG was performed to rule out any heart abnormalities in the context of possible caffeine toxicity. Sixty participants were recruited in eight sessions in two experiments; 30 caffeine-naïve subjects for Experiment 1 (15 male; age $23.6 \pm 3.3$ years (mean \pm SD, range 19-31), and 30 caffeine consumers for Experiment 2 (15 male; age 23.8 \pm 2.3 years (mean $\pm \mathrm{SD}$, range 19-29). The latter had been instructed to abstain from all caffeinated

products on the day of the experiment. All were right-handed as assessed by the Edinburgh handedness inventory (Oldfield, 1971). Their medication history, and consumption of alcohol and caffeinated products were recorded in every session. The sessions with female participants took place in the period between menstruations (at least five days after the menses ended) in order to ensure constant oestrogen levels (Smith et al., 1999; Lee et al., 2018). This study was approved by the local ethics committee and conducted in accordance with the Declaration of Helsinki. It was registered in ClinicalTrials.gov with the registration IDs NCT03720665 and NCT04011670.

\subsubsection{Transcranial Magnetic Stimulation (TMS)}

Single-pulse TMS was used to induce motor potentials (MEPs) in the right first dorsal interosseous muscle (FDI) that were recorded with surface $\mathrm{Ag}-\mathrm{AgCl}$ electrodes in a belly-tendon montage. TMS was performed with a Magstim 200² magnetic stimulator (Magstim Co. Ltd., Whitland, Wales, 
$\mathrm{UK}$ ) and a D70 coil. The location $\mathrm{Cz}$, the intersection of the line between nasion and inion, and that between left and right periauricular, was marked and used as a landmark for positioning the coil in following sessions. The coil was held tangentially to the skull in a posterior-anterior (PA) direction at a $45^{\circ}$ angle pointing towards the right forehead. The electromyography signals were amplified, band-pass filtered $(2 \mathrm{~Hz}-2 \mathrm{kHz})$, and digitised at a sampling rate of $5 \mathrm{kHz}$ with a micro 1401 AD converter (Cambridge Electronic Design Ltd., Cambridge, UK). All data were stored on a hard disc for off-line analysis. A customised script was used to extract peak-to-peak amplitudes (Signal software version 4; Cambridge Electronic Design Ltd., Cambridge, UK). In each session, we determined the resting motor threshold (RMT), i.e. the lowest stimulus intensity required to elicit peak-to-peak MEPs of $\geq 50 \mu \mathrm{V}$ in five of ten stimulations. The intensity required to produce approximately $1 \mathrm{mV}$ peak-to-peak MEPs $\left(\mathrm{MT}_{1 \mathrm{mV}}\right)$ from 25 stimuli was determined following the protocols described in (Rossini et al., 2015).

\subsubsection{Paired associative stimulation (PAS)}

The right median nerve was stimulated electrically using two ECG electrodes. The anodal electrode was attached over the median nerve at the wrist and the cathodal electrode $2 \mathrm{~cm}$ more proximally. The TMS coil was positioned over the left M1 in the same way as for single-pulse TMS and was held in a coil stand. In the PAS protocol, the median nerve was stimulated with 90 non-painful, constant current, $0.2 \mathrm{~ms}$ square pulses (pulse generator DS5, Digitimer, UK) at a frequency of $0.05 \mathrm{~Hz}$ in a series lasting 30 minutes (Stefan et al., 2000, 2002). Each electrical stimulus was followed by a single-pulse TMS with an inter-stimulus interval (ISI) of $25 \mathrm{~ms}$. The electrical stimulation current was $300 \%$ of the participant's perceptual threshold. This was determined before beginning the session by increasing the stimulation current until the first faintest sensation was perceived.

\subsubsection{Transcranial Alternating Current Stimulation (tACS)}

Transcranial AC stimulation with a $140 \mathrm{~Hz}$ sinusoidal waveform was delivered by a battery-driven stimulator (NeuroConn GmbH, Illmenau, Germany) through two conductive rubber electrodes. The active electrode $(4 \times 4 \mathrm{~cm})$ was placed over the left primary motor cortex at the position giving 
the best MEP response in the target FDI (M1), and the return electrode $(5 \times 7 \mathrm{~cm})$ was placed over the contralateral orbitofrontal cortex with the long edge above and parallel to the right eyebrow. The electrode was oriented so that the cable exiting from the short side led around to the right, while the cable of the M1 electrode was led to the back. The electrodes were prepared following the original protocol of Moliadze and colleagues (Moliadze, Antal and Paulus, 2010; Moliadze et $a l ., 2012)$ as closely as possible in order to replicate their findings. We used electrode cream for the M1 electrode (Ten20, D.O. Weaver, Aurora, CO, USA) and a saline soaked sponge for the return electrode. The stimulation duration was ten minutes with a current intensity of either $1 \mathrm{~mA}$ or $0.4 \mathrm{~mA}$. The current was ramped up over five seconds at the beginning of stimulation and maintained for ten minutes. At the end of stimulation, it was ramped down over five seconds and immediately terminated. In the sham stimulation, the current was ramped up over five seconds and maintained the stimulation for 30 seconds before being ramped down over five seconds. This 30second stimulation was intended to induce a similar skin sensation for the purpose of blinding. The impedance was kept below $10 \mathrm{k} \Omega$. The participants filled out questionnaires regarding stimulationrelated sensations after each session.

\subsubsection{Pupillometry}

We measured pupillary oscillations to assess drowsiness and alertness with a pupillometer (F2D, AMTech Pupilknowlogy GmbH, Dossenheim, Germany). The pupillometer goggles seal out ambient light and measure pupillary motion in total darkness with infrared video cameras. The measurements were performed with the participant seated in a comfortable chair. The pupillometer goggles were donned after the tACS electrodes had been attached, and the experimenter made sure that the light seal of the goggles was complete. The participants were instructed to sit quietly, keep their eyes open and fixate a green dot, and try to avoid frequent blinking. The infrared cameras continuously monitored pupil diameter (PD). The duration of the pupillometry session in the dark with recoding of the spontaneous pupillary oscillations was 11 minutes. The session began with an unstimulated one-minute dark adaptation period followed by ten minutes with tACS. We used the pupillary unrest index (PUI), a measure of spontaneous pupillary oscillations, as an indicator for alertness during a stimulation. The PUI is defined by cumulative changes of the pupil diameter based on mean values of 11 minutes data recording (Lüdtke et al., 1998). It indicates the amount 
of change of the PD in $\mathrm{mm} / \mathrm{min}$. A higher PUI score reflects an increase in daytime sleepiness or reduced alertness (Regen, Dorn and Danker-Hopfe, 2013). Methodological details of this technique and data processing are described in (Lüdtke et al., 1998).

\subsubsection{Study design}

This study encompassed two randomised, double-blind, cross-over designed sub-studies. The treatment orders for both medication and stimulation for each participant were randomly assigned by a statistician who was not involved in the study. Before each session the participants filled out the questionnaire described above.

Experiment 1 was designed to evaluate the influence of caffeine on cortical plasticity generated by $140 \mathrm{~Hz}$ tACS and $25 \mathrm{~ms}$ PAS (Figure 3.1a). The participant was instructed to sit comfortably in a reclining chair, and the experiment began with the determination of the optimal position of the TMS coil (see above). The position was marked and was used as a guide for reproducibly positioning the coil. The resting motor threshold (RMT) was determined with the coil over the identified optimal position following the guidelines of (Rossini et al., 2015). In the next step, we increased the stimulation intensity until a consistent MEP of approximately $1 \mathrm{mV}$ (peak to peak amplitude) $\left(\mathrm{MT}_{1 \mathrm{mV}}\right)$ was achieved. This intensity was used to record the baseline MEPs (PRE).

Intervention 1 started with the administration of a tablet containing either caffeine or placebo followed by a 45-minute waiting period to allow for drug uptake. Peak saliva caffeine level have been reported to appear later after a capsule than after coffee or cola (capsule peak time : $67 \pm 7$ min, mean \pm SD; coffee peak time: $42 \pm 5$ min, mean \pm SD) (Liguori, Hughes and Grass, 1997). During this period, the participants were allowed to read supplied materials, which were chosen to maintain wakefulness but not provoke arousal. Immediately after the 45-minute waiting period, Intervention 2 (tACS or PAS 25) was administered following the randomised stimulation protocols. The excitatory and inhibitory tACS protocols lasted for ten minutes, while the PAS 25 protocol was administered for 30 minutes for each protocol according to the randomisation order. 
Aftereffects were measured by stimulating the designated cortical area. Poststimulation effects were recorded at $0,5,10,15,20,25$, and 30 minutes after the end of stimulation (Figure 3.1a).

Experiment 2 (Figure 3.1b) was designed to determine the influence of time of day with or without caffeine on tACS on the plasticity aftereffects. The study protocols all followed the same schedule as in Experiment 1 except that we changed the stimulation regimen in Intervention 2. It consisted in a combination of two stimulation and two time of day conditions in a randomized order: i) tACS (1.0 mA); morning, ii) tACS (1.0 mA); afternoon, iii) tACS (Sham); morning, iv) tACS (Sham); afternoon.

In both the morning and afternoon sessions, the pupillary oscillations were monitored, and the participants were notified whenever changes in the PUI indicated a reduction in alertness and eyelids closure. The experimenter had to ensure that the participants opened their eyes to obtain a valid measurement. Participants were tested at the same time for each morning and afternoon session; morning start time 09:41 \pm 0:49 (mean \pm S.D, range 07: 35 - 11:33); afternoon start time 15:07 \pm 1:05 (mean \pm S.D, range 12:00 - 18:03). The sessions were performed blinded in a randomised order with at least five days between sessions to avoid carryover effects. 
a)

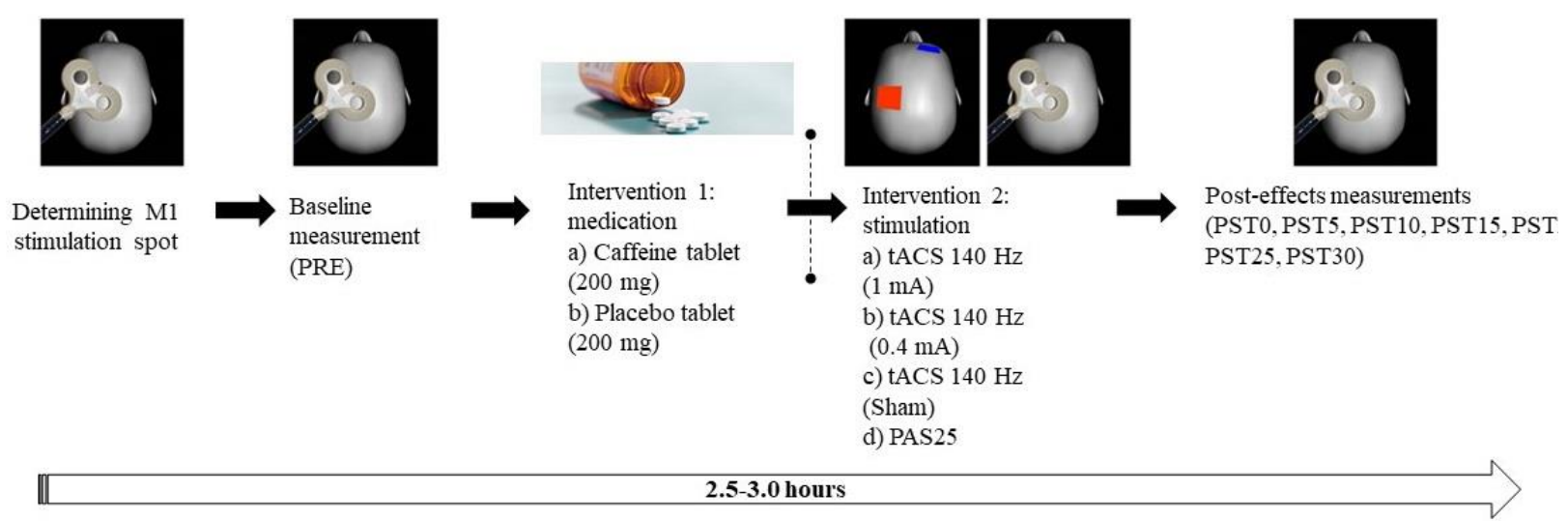

$\bullet--45 \mathrm{~min}$ pause

b)

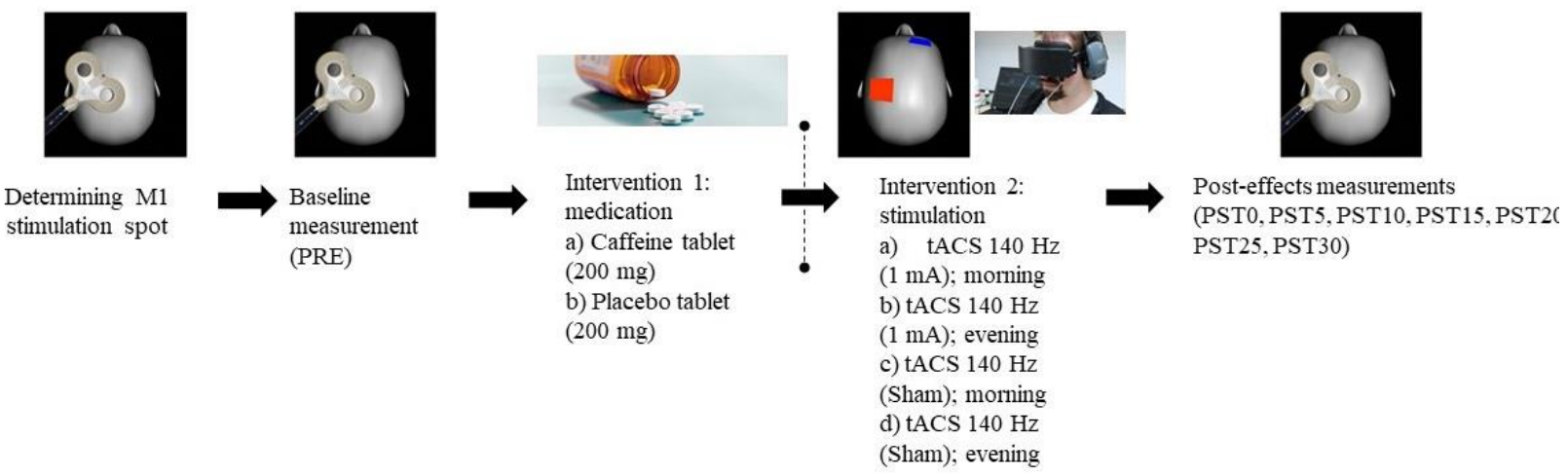

Figure 3.1 Experimental design.

The optimal stimulation position over the left motor cortex (M1) for the right FDI muscle was located and baseline measurements of the induced MEPs were performed. The caffeine or placebo tablets were administered in a random order then 45 -minute wait for drug uptake (Intervention 1). 
Experiment 1, studied the effect of caffeine on transcranial stimulation-induced plasticity in caffeine-naïve participants $(n=30)$.

Intervention 2: Ten minutes of $140 \mathrm{~Hz}, 1.0 \mathrm{~mA}$ tACS or sham tACS, or 30 minutes of PAS 25, was applied to the defined M1 location. Beginning immediately after stimulation, the post stimulation effects were measured at five-minute intervals for 30 minutes.

Experiment 2 studied the influence of time of day and alertness during stimulation on tACS aftereffects in caffeine consumers $(\mathrm{n}=30)$

Preparation as in Experiment 1. $140 \mathrm{~Hz}, 1 \mathrm{~mA}$ tACS or sham stimulation was performed. The level of alertness was measured by pupillometry during the stimulation. The morning sessions were conducted before 12:00, and the afternoon sessions were conducted after 12:00. The post stimulation measurements were as in Experiment 1.

The order of the sessions in both Experiment 1 and 2 were randomized with at least five days between each session. Abbreviations: $\mathrm{MEP}=$ motor evoked potential; $\mathrm{tACS}=$ transcranial alternating current stimulation; PAS = paired associative stimulation; M1 = primary motor cortex; $\mathrm{PRE}=$ baseline PST $=$ post stimulation .

\subsubsection{Data analysis and statistics}

A customised Signal software script was used to evaluate the peak-to-peak MEP amplitudes in each trial in each session (Signal version 4.08, Cambridge Electronic Design Ltd., Cambridge UK). At each measurement time, the amplitudes of 25 MEPs were averaged and stored by the SPSS software for statistical analysis (IBM SPSS statistics 26; IBM Corp., Armonk, NY, USA). The data were coded according to the stimulation condition and were labelled according to the measurement times $($ Baseline $=\mathrm{PRE}$, Post $0 \mathrm{~min}=\mathrm{PST} 0$, Post $5 \mathrm{~min}=\mathrm{PST} 5$, Post $10 \mathrm{~min}=$ PST10, Post $15 \mathrm{~min}=\mathrm{PST} 15$, Post $20 \mathrm{~min}=\mathrm{PST} 20$, Post $25 \mathrm{~min}=\mathrm{PST} 25$ and Post $30 \mathrm{~min}=$ PST30). The first four post measurements were pooled to give the first 15 minutes post data point (POST 1: PST0 - PST15), later the last three post measurements were pooled to give the post data point after 15 minutes (POST 2: PST20 - PST30). The percent coefficient of variation (\% CV), i.e. standard deviation of the subset times 100 divided by mean of the subset was used to describe intra-session variability for each participant across sessions (Biabani et al., 2018). The mean pupillary unrest index (PUI) data given in $\mathrm{mm}$ per $\min \left(\mathrm{mm} * \mathrm{~min}^{-1}\right)$ was $\log _{10}$-transformed for further parametric analysis. Data quality was assessed by manual inspection, and we excluded recordings in which more than $50 \%$ of the data was missing (i.e. half of the data were interpolated) as described in Regen et al. (Regen, Dorn and Danker-Hopfe, 2013). 
The Shapiro-Wilk test was used to test data distribution, and non-parametric tests were used if the normality assumption was violated. A chi-square test was used to compare the perceive sensation and the rating of stimulation type between stimulation conditions. A repeated measures (rm) ANOVA was used to compare RMT, MT $1 \mathrm{mV}$ and baseline MEP amplitude (MEPPRE) between sessions. In Experiment 1, we examined the group differences on RMT, MT $1 \mathrm{mV}$ and MEP $\mathrm{PRE}$ with stimulation and drug as within-subjects factor (STIMULATION 4 levels $x$ DRUG $_{2}$ levels). Similarly to Experiment 2, we examined the group differences with stimulation, day and drug as within-subject factor (STIMULATION 2 levels $\mathrm{x} \mathrm{DAY}_{2}$ levels $\mathrm{x} \mathrm{DRUG}_{2}$ levels).

In Experiment 1, a rmANOVA was used for the PAS 25 datasets to evaluate the effects of caffeine on cortical excitability with time and drug as within-subject factors (TIME 8 levels $\mathrm{x} \mathrm{DRUG}_{2}$ levels). The two-tailed paired Student t-test was used to compare baseline (PRE) with the pooled datasets (POST 1 and POST 2). Two rmANOVA analyses of the tACS datasets were performed. The first was to evaluate the effects of caffeine where time, stimulation and drug were the withinsubject factors $\left(\mathrm{TIME}_{8}\right.$ levels $\mathrm{x}$ STIMULATION 3 levels $\mathrm{x} \mathrm{DRUG}_{2}$ levels). The second rmANOVA was to evaluate the effects of PRE on the pooled datasets (POST 1 and POST 2) (TIME 3 levels $\mathrm{x}$ STIMULATION $_{3}$ levels $x$ DRUG $_{2}$ levels). $R$ m ANOVAs were also used to compare the aftereffects of cortical excitability in Experiment 2. We included time, stimulation, drug and time of day as within-subject factors (TIME 8 levels $\mathrm{x}$ STIMULATION 2 levels $\mathrm{X} \mathrm{DRUG}_{2}$ levels $\mathrm{X} \mathrm{DAY}_{2}$ levels). We also did rmANOVA to evaluate the effects of PRE on the pooled datasets (POST 1 and POST 2) in Experiment 2.

The Wilcoxon signed rank test was used to compare the variability between sessions with caffeine or placebo (inter-session variability) using the coefficient of variation (CV) of POST MEP amplitudes in all stimulation conditions. The differences were reported as median and interquartile range (IQR). The average alertness score from three visual analogue scale (VAS) data sets (PST0, PST15, PST30) was calculated to evaluate the difference in the participants' alertness during post measurement effects with MEP changes. A paired Student t-test was used to compare the alertness level in sessions with caffeine or placebo under each stimulation condition. 
An independent t-test was used to compare the mean differences of RMT, $\mathrm{MT}_{1 \mathrm{mV}}$ and

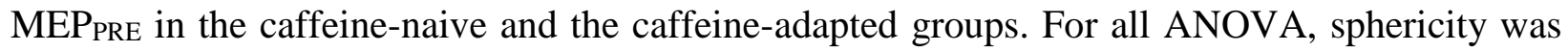
assessed with Mauchly's test of sphericity, and Greenhouse-Geisser correction was used if sphericity was violated. The Bonferroni correction was used for multiple comparisons, and subsequent post hoc comparisons were performed with paired t-tests. All data were presented as mean \pm standard error of mean (SEM), unless otherwise specified. The significance level was set at $\mathrm{p} \leq 0.05$.

\subsection{RESULTS}

\subsubsection{Motor threshold and baseline MEP (MEP}

In Experiment 1, the motor thresholds (RMT and $\left.\mathrm{MT}_{1 \mathrm{mV}}\right)$ did not differ significantly between the sessions. The baseline MEP amplitudes (MEP PRE) were the same in all sessions. (Supplementary Table 3.1).

The mean self-reported alertness scores in the post stimulation periods were significantly higher after caffeine intake except in the session with $1 \mathrm{~mA}$ tACS, where the score was higher with caffeine but the difference did not reach statistical significance. (Table 3.1).

Table 3.1 Self-reported alertness scores (PST0 to PST30)

\begin{tabular}{llll}
\hline & Alertness score & $\mathbf{t}$ & $\mathbf{p}$ \\
\hline tACS & & & 0.26 \\
1) $1.0 \mathrm{~mA}$; placebo & $6.9 \pm 1.4$ & -1.14 & \\
2) $1.0 \mathrm{~mA}$; caffeine & $7.2 \pm 1.5$ & & $0.006^{*}$ \\
3) $0.4 \mathrm{~mA}$; placebo & $6.7 \pm 1.4$ & -2.97 & \\
4) $0.4 \mathrm{~mA}$; caffeine & $7.3 \pm 1.4$ & & $0.02^{*}$ \\
5) Sham; placebo & $6.7 \pm 1.2$ & -2.45 & \\
6) Sham; caffeine & $7.5 \pm 1.5$ & & $0.03^{*}$ \\
PAS25 & & & \\
1) Placebo & $6.6 \pm 1.5$ & -2.31 &
\end{tabular}


In Experiment 2, the motor thresholds (RMT and $\mathrm{MT}_{1 \mathrm{mV}}$ ) did not differ significantly between the two groups in any session. The baseline MEP amplitudes (MEPPRE) of both groups were similar in all four settings (Supplementary Table 3.2).

\subsubsection{Stimulation perception and correct identification of stimulation type}

Some participants reported cutaneous sensations, like itching, pain, burning, or heat under the stimulation electrode. In Experiment 1 the numbers of reported sensations were significantly lower (Yes: 43.3\%; No: 56.7, $\chi 2(5)=20.63, \mathrm{p}<0.05)$. There were no differences of reported sensations between stimulation conditions in both ratings. There were no significant differences in the number of participants who correctly identified the type of stimulation in any of the three comparisons (True: $32.8 \%$, Placebo: 37.2\%, Don't know: 30\%; $\chi 2(10)=9.96, p>0.05)$. (Supplementary Table 3.3).

In Experiment 2, non-significant results were seen both with regard to reports of cutaneous sensations (Yes: 42.1\%; No: 57.9\%; $\left.\chi^{2}(7)=4.95, \mathrm{p}>0.05\right)$ and with correct identification of stimulus type (True: 40.6\%, Placebo: 30.3\%, Don't know: 29.1\%: $\chi^{2}(14)=11.81, \mathrm{p}>0.05$ ). (Supplementary Table 3.4).

\subsubsection{Experiment 1: Effects of caffeine on PAS 25 and LTP/LTD-like aftereffects after $140 \mathrm{~Hz} \mathrm{tACS}$ in caffeine-naïve participants}

There was a long lasting increase in the MEP amplitudes after PAS 25 which was greater with caffeine than with placebo (Figure 3.2a). This is also seen in the analysis of the pooled post stimulation data that showed up to $64.0 \pm 9.0 \%$ increase over baseline after caffeine compared with up to $26.1 \pm 9.1 \%$ change after placebo (Figure 3.2b). Significant factors were TIME (p < 0.001) and DRUG ( $<$ 0.05). There was a significant interaction between TIME and DRUG on the plasticity aftereffects induced by PAS 25 ( $\mathrm{p}<0.05)$. (Supplementary Table 3.5 ). 


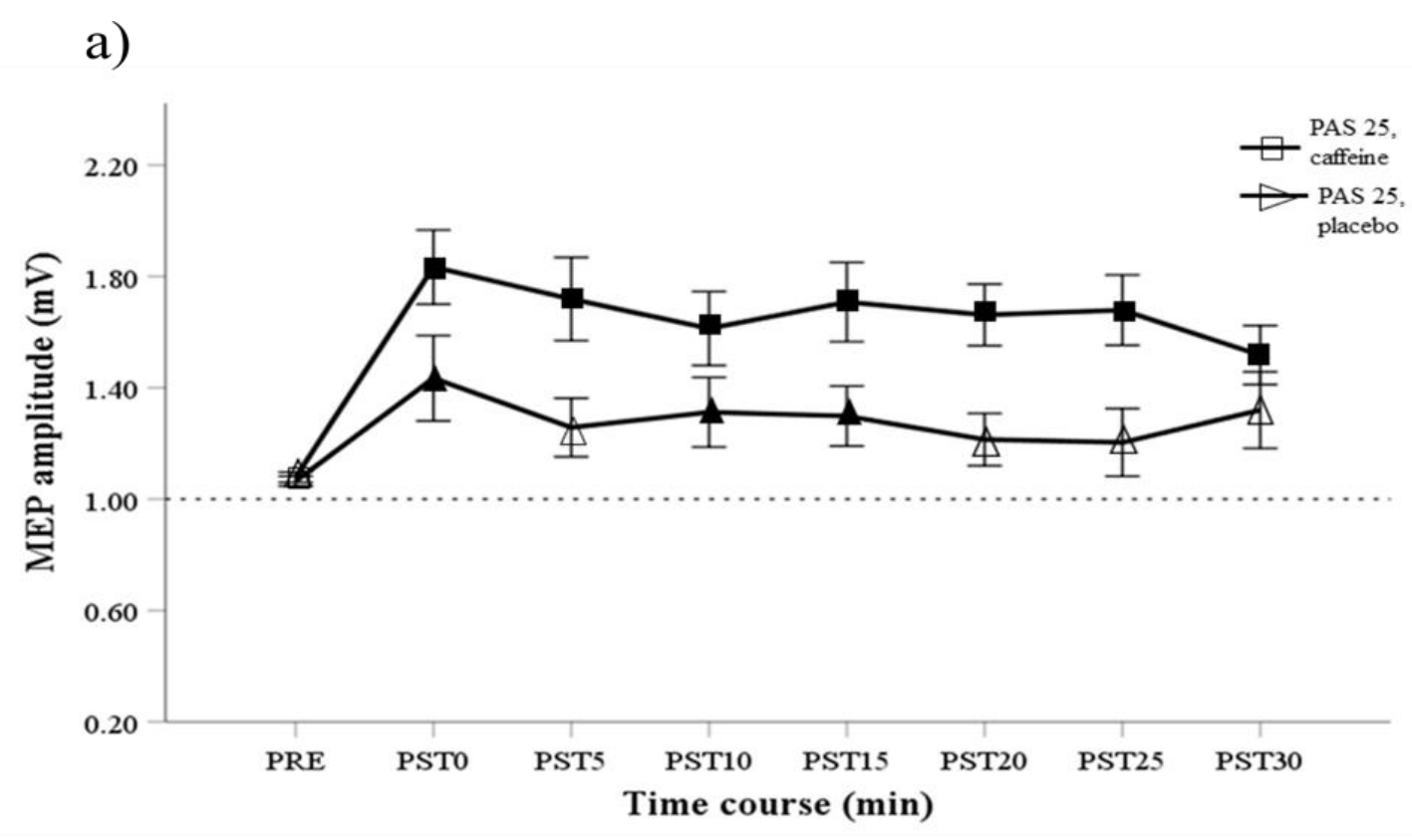

b)

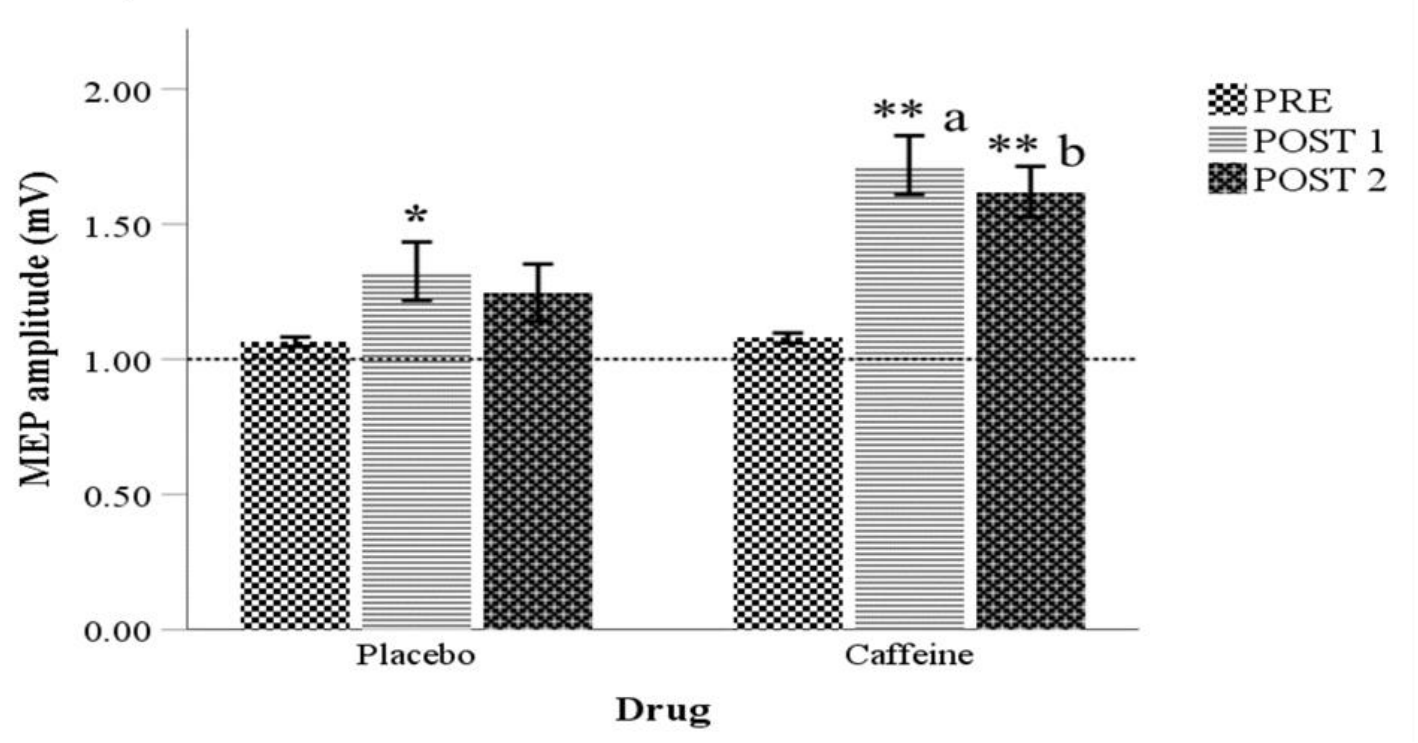

Figure 3.2 Effect of caffeine on plasticity aftereffect following PAS 25 (Experiment 1).

a) The post stimulation MEPs were increased above baseline in both groups, significantly also at various time points (filled symbols).

b) Comparison of pooled post stimulation data. POST 1 MEP amplitude after placebo was significantly higher compared to baseline $(\mathrm{t}(29)=-2.53, \mathrm{p}=0.02)$. MEP facilitation after caffeine was significantly higher at POST 1 compared to baseline $(\mathrm{t}(29)=-5.85, \mathrm{p}<0.001)$ and was significantly higher at POST 2 compared to baseline $(\mathrm{t}(29)=-5.73, \mathrm{p}<0.001)$. POST 1 MEPs after 
caffeine was significantly higher than after placebo $(t(29)=-3.09, p=0.004)$. POST 2 MEPs after caffeine was significantly higher than after placebo $(\mathrm{t}(29)=-2.62, \mathrm{p}=0.01)$

Data are shown as means \pm SEM; filled symbols indicate a significant difference from baseline; $* \mathrm{p}$ $<0.05 ; * * * \mathrm{p}<0.001 ; \mathrm{a}=$ significant difference of POST 1 between placebo and caffeine sessions; $\mathrm{b}=$ significant difference of POST 2 between placebo and caffeine sessions

Abbreviations: $\mathrm{MEP}=$ motor evoked potential; $\mathrm{PAS}=$ paired associative stimulation; $\mathrm{PRE}=$ baseline; PST = post stimulation; POST $1=$ average of first 15 min post stimulation measurements; POST $2=$ average of last 15 min post stimulation measurements.

Caffeine (DRUG) had a significant effect $(\mathrm{p} \leq 0.05)$ on the cortical excitability changes in the tACS protocols, but no significant effects were detected for the factors TIME and STIMULATION (Supplementary Table 3.5). A borderline significant main effect of DRUG indicated MEP facilitation after caffeine administration. (See Figure 3.3a - Figure 3.3c).

Analysis of the pooled datasets showed that the post stimulation change of MEPs from baseline (in percent) were greater with caffeine than with placebo. A separate rmANOVA of the PRE and POST data points showed an interaction effect for TIME x DRUG $(p<0.05)$. No other significant factors or interactions were detected (Figure 3.3d and Supplementary Table 3.5). There was no inter-session variability between session with caffeine and placebo (Supplementary Figure $3.1)$.

To summarise, caffeine enhanced the plasticity aftereffects after PAS 25, and although it generally increased cortical excitability after tACS. The lack of MEP facilitation after tACS with placebo emphasizes the fact that caffeine, and not stimulation, was the responsible factor. 
a)

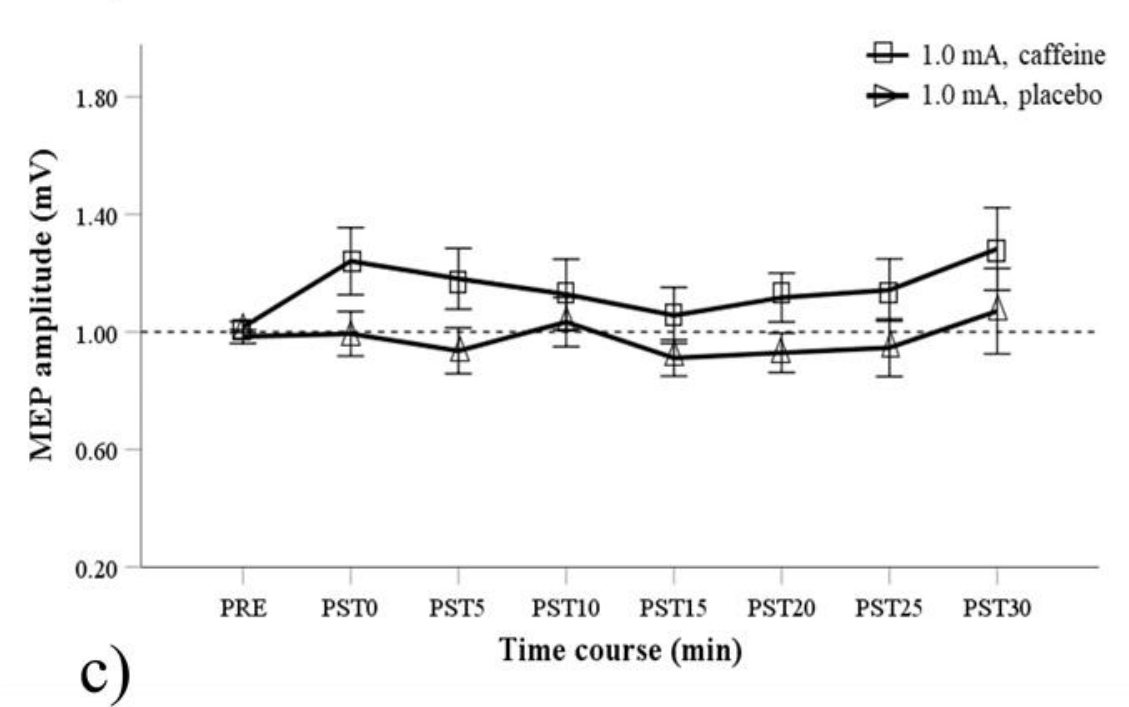

c)

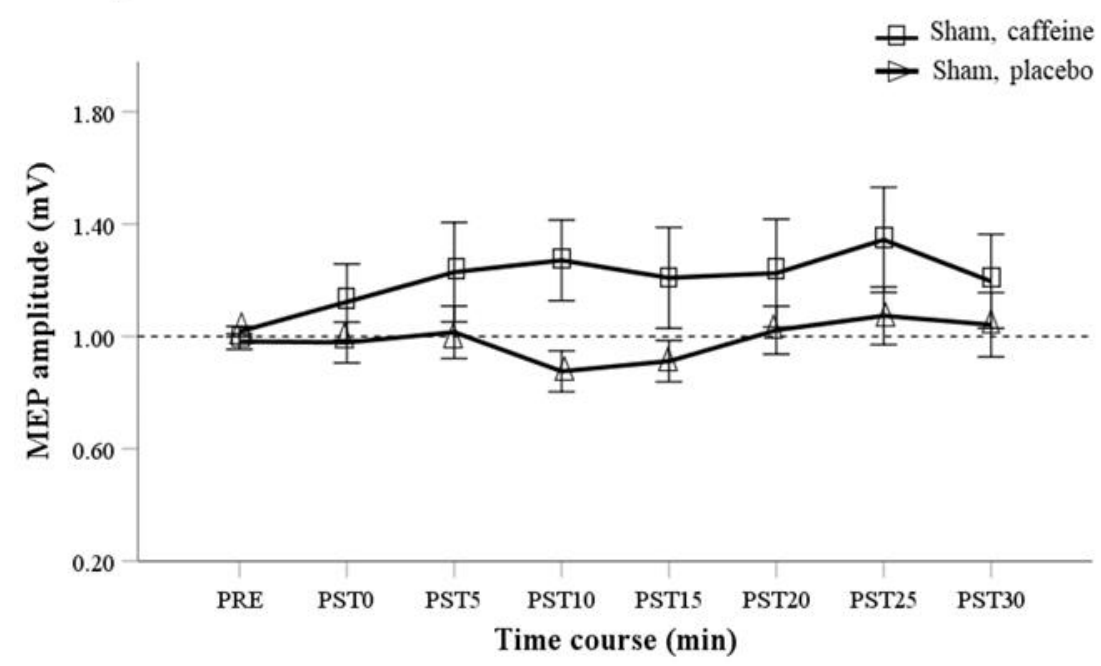

b)

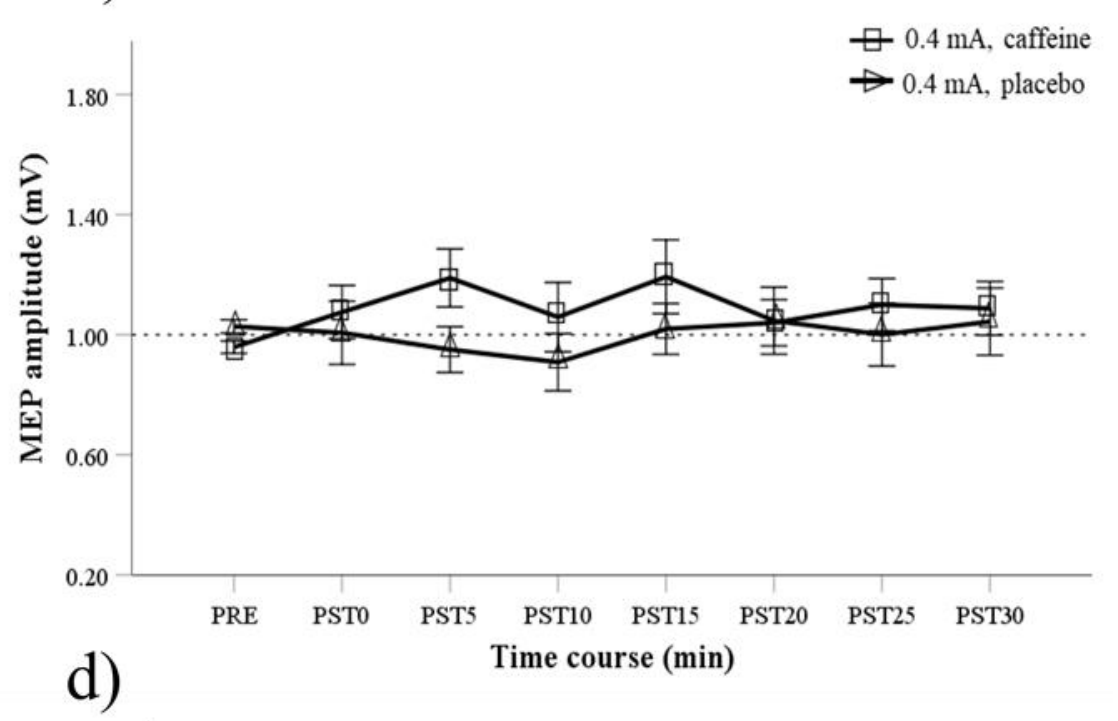

Figure 3.3 Cortical excitability after tACS with or without caffeine (Experiment 1).

MEP amplitudes after tACS with different stimulation intensities: a) $1.0 \mathrm{~mA}$; b) $0.4 \mathrm{~mA}$, and c) Sham. 
d) Analysis of pooled data revealed the interaction effects of drug and time $(\mathrm{p}=0.04)$. Caffeine induced a general facilitatory increase of MEP amplitudes in both post measurement time points. This effect was significantly greater after caffeine than after placebo at POST $2(\mathrm{t}(29)=-2.03$, $\mathrm{p} \leq 0.05)$ in the sessions with $1.0 \mathrm{~mA}$ tACS

Data are shown as means \pm SEM, a $=$ significant difference between placebo and caffeine sessions. Abbreviations: $\mathrm{MEP}=$ motor evoked potential; tACS = transcranial alternating current stimulation; PRE = baseline; PST = post stimulation; POST $1=$ average of first 15 min post stimulation measurements; POST 2 = average of last 15 min post stimulation measurements 


\subsubsection{Experiment 2: Effects of caffeine, time of day and alertness during stimulation on cortical excitability in the caffeine consumer group}

The main effect factors were TIME and STIMULATION with a significant interaction effect between TIME x STIMULATION x DAY. (Supplementary Table 3.6). Cortical excitability was lower than baseline at various timepoints and in various sessions, particularly after sham stimulation, morning. In the morning sessions the MEP amplitudes were greater after $1.0 \mathrm{~mA} \mathrm{tACS}$ than after sham stimulation (See Figure 3.4a). In the caffeine sessions the MEP amplitudes decreased significantly immediately after sham stimulation in the morning and afternoon sessions (See Figure 3.4b). Analysis of the pooled data revealed that the main effect factors were TIME and STIMULATION with significant interaction effects between TIME x STIMULATION and STIMULATION x DAY x DRUG. (Supplementary Table 3.7). The MEP amplitudes were always significantly reduced during the first 15 min post measurements (POST 1) except after $1 \mathrm{~mA}$ tACS in the morning session. (See Figure 3.4c). In the placebo, morning sessions 1mA tACS prevented the MEP decrease seen after sham stimulation. Both time points showed the post stimulation MEP amplitudes after $1 \mathrm{~mA}$ tACS, placebo in the morning were significantly higher than MEPs after sham stimulation, caffeine in the afternoon. (See Figure 3.4c). 

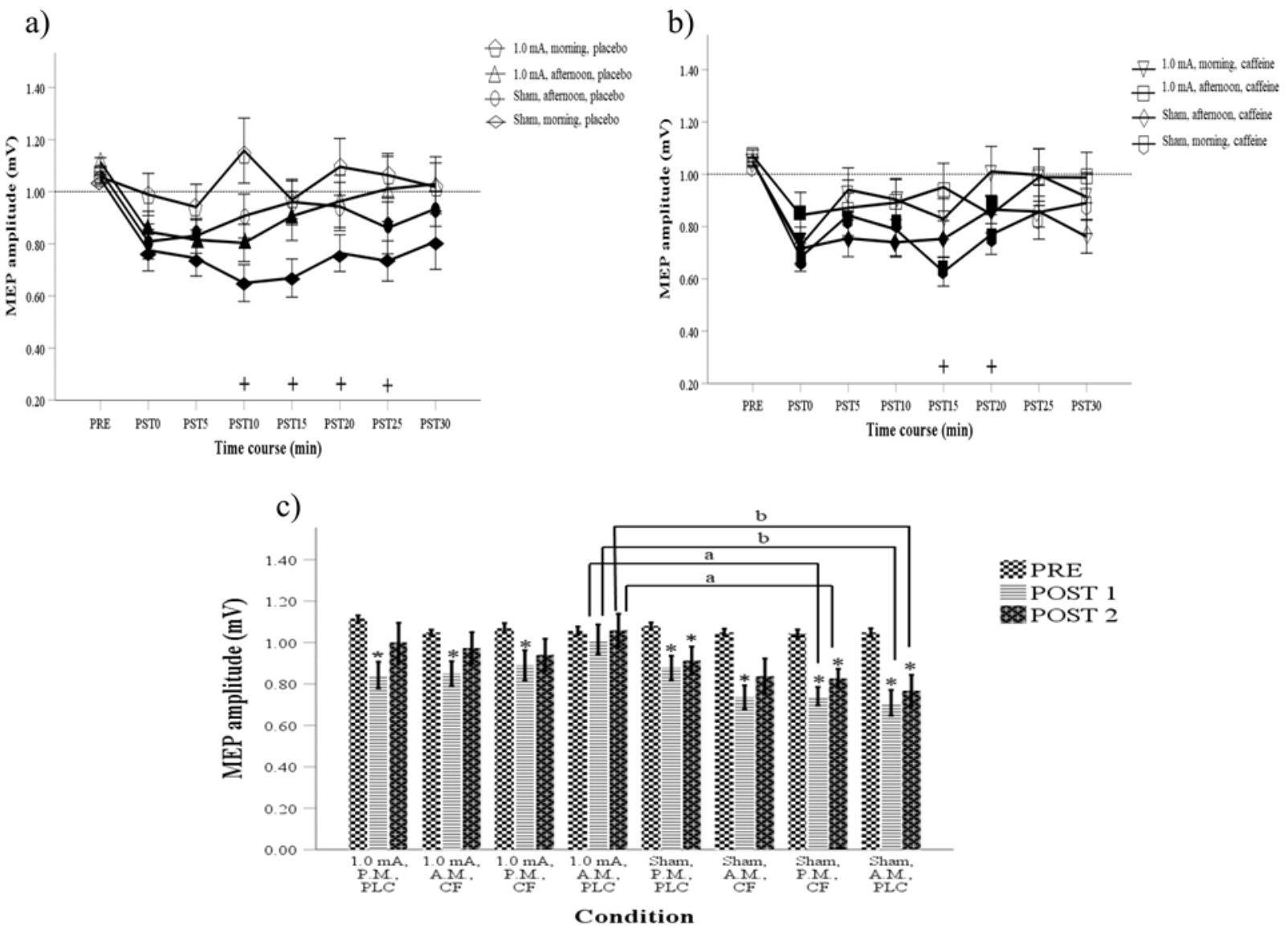

Figure 3.4 Cortical excitability with placebo or caffeine (Experiment 2).

a) Time course of MEP amplitudes after actual or sham tACS in the placebo sessions. MEP amplitudes were reduced for 30 minutes in some sessions. Cortical excitability in sham, morning were significantly lower at PST10 - PST25 than $1.0 \mathrm{~mA}$ tACS, morning

b) With caffeine, the MEP amplitudes were reduced for 20 minutes immediately following stimulation and MEPs in sham, morning were significantly lower at some points than sham, afternoon.

c) Analysis of pooled data of all data points in each session showed significant effects of time (p < $0.001)$ and stimulation $(\mathrm{p}=0.002)$ with interaction effects of time and stimulation $(\mathrm{p}=0.031)$, and stimulation and time of day and drug $(\mathrm{p}=0.045)$ on cortical excitability were observed. MEP amplitudes were reduced during the first 15 min post measurements in all sessions $(\mathrm{p}<0.05)$ except a session after $1.0 \mathrm{~mA} \mathrm{tACS}$, placebo in the morning. MEP amplitudes were significantly higher after $1.0 \mathrm{~mA}$ tACS in the morning, placebo than after sham stimulation in the afternoon, caffeine at both time points (POST 1: $\mathrm{t}(29)=3.18, \mathrm{p}=0.004$; POST 2: $\mathrm{t}(29)=2.42, \mathrm{p}=0.02)$. In the morning, placebo sessions, the MEPs were significantly higher after $1.0 \mathrm{~mA}$ tACS than after sham at both time points (POST 1: $\mathrm{t}(29)=3.18, \mathrm{p}=0.004$; POST 2: $\mathrm{t}(29)=2.49, \mathrm{p}=0.02)$.

Data are presented as means \pm SEM. Filled symbols indicate significant difference from baseline; a = significant difference between $1.0 \mathrm{~mA}$ tACS in the morning, placebo and sham stimulation in the afternoon caffeine; $b=$ significant difference between actual or sham tACS in the morning, placebo sessions; $+=$ significant difference between $1.0 \mathrm{~mA}$ and sham stimulation in morning placebo 
sessions; $* \mathrm{p}<0.05$; POST $1=$ average of the first 15 min post stimulation measurements; POST 2 $=$ average of the last 15 min post stimulation measurements; A.M. = morning; P.M. = afternoon; $\mathrm{PLC}=$ placebo; $\mathrm{CF}=$ caffeine.

\subsubsection{Alertness and cortical excitability}

Six pupillometry datasets had to be discarded due to poor data quality; two in the $1.0 \mathrm{~mA}$, morning, placebo session, two in the Sham, afternoon, placebo session, and two in the Sham, morning, placebo session. Pupillary oscillations were reduced after caffeine intake indicating that the participants were more alert (Figure 3.5a and Supplementary Table 3.8).

Mean pupil diameter was slightly, but not significantly, larger after caffeine administration. This indicates that PD is not a sensitive measure of alertness or daytime sleepiness. It also varies according to the time of the day (Eggert et al., 2012). (Figure 3.5b and Supplementary Table 3.8).

There was a small, but significant, negative correlation between the natural log transformed PUI data and the post stimulation MEP amplitudes. Seeing that PUI values and level of alertness are negatively related this correlation showed a positive effect of increased alertness during tACS on post stimulation excitability changes in the motor cortex (See Figure 3.6a). There were no significant correlations between alertness and plasticity aftereffects in the caffeine sessions (See Figure 3.6b - Figure 3.6d).

To sum up, in caffeine consumers, MEPs were higher after tACS than after sham stimulation. Caffeine and time of day did not influence the plasticity aftereffects induced by tACS. Light-deprivation during stimulation led to a reduction in the post stimulation MEP amplitudes. 
a)

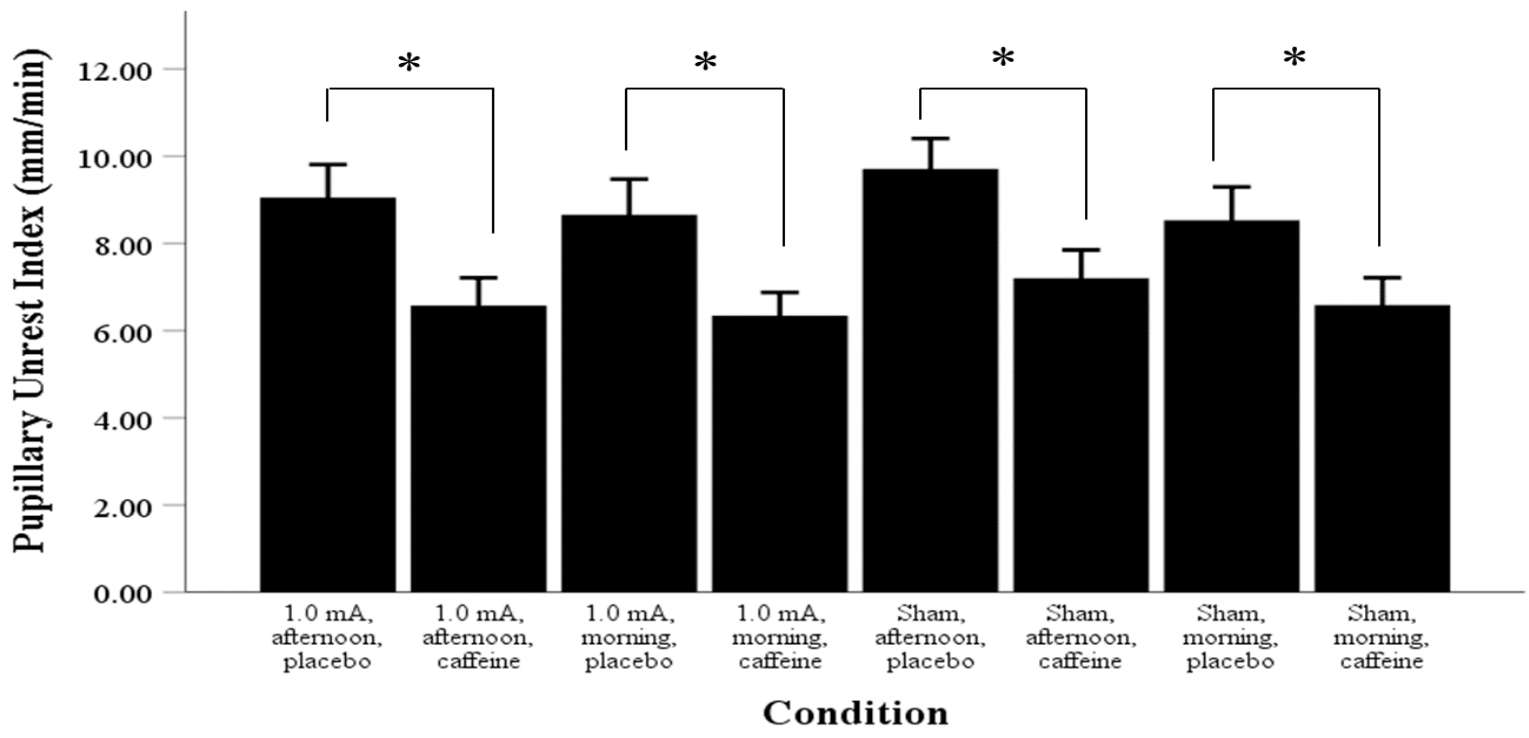

b)

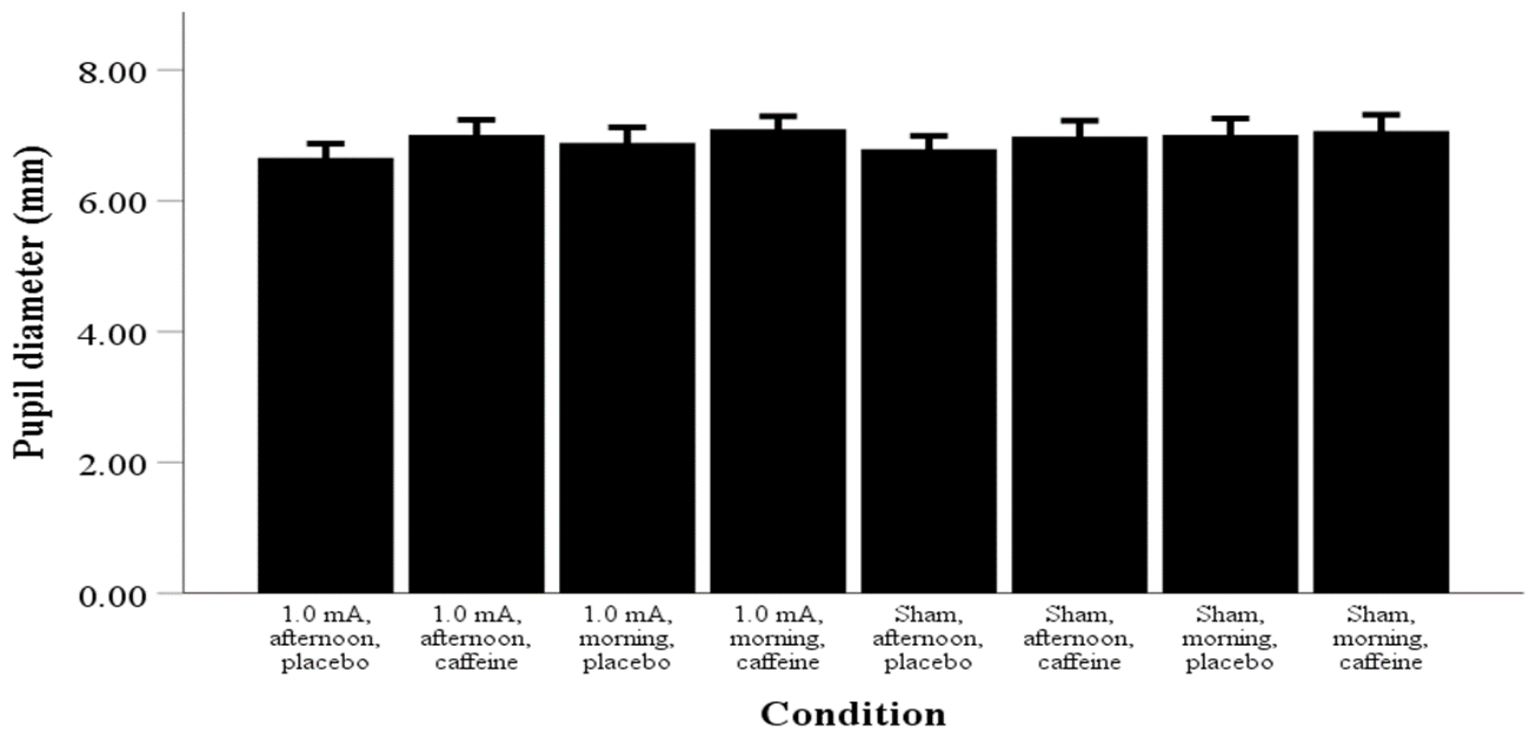

Figure 3.5 Pooled data for pupillary oscillations (PUI) and mean pupil diameters during stimulation (Experiment 2). 
a) Pupillary Unrest Index (PUI) as an objective measure of alertness showed that the participants were more alert after caffeine administration than after placebo $(\mathrm{p}<0.05)$ as indicated by less pupillary fluctuation.

b) Mean pupil diameters did not differ in any session with or without caffeine.

Data are reported as means $\pm \mathrm{SD} ; * \mathrm{p}<0.05$.

a)

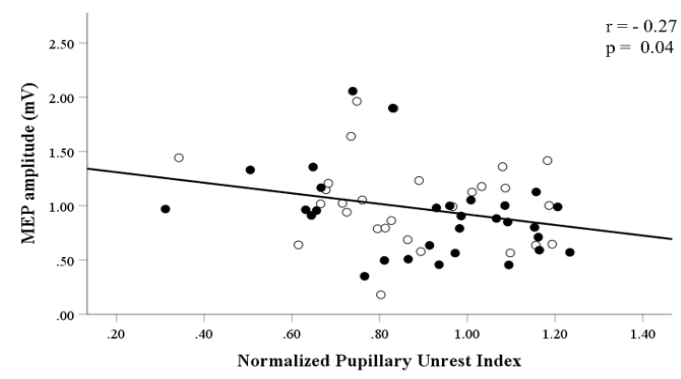

c)

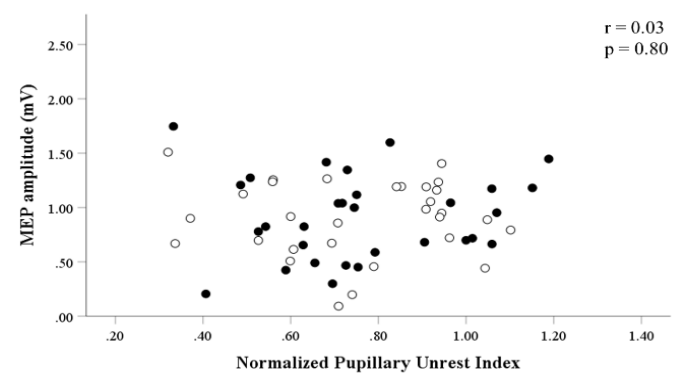

b)

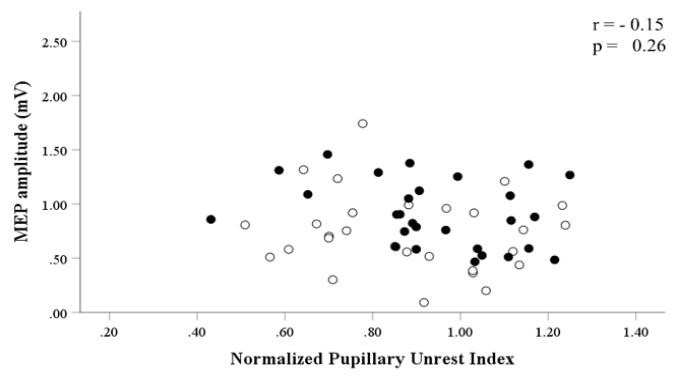

d)

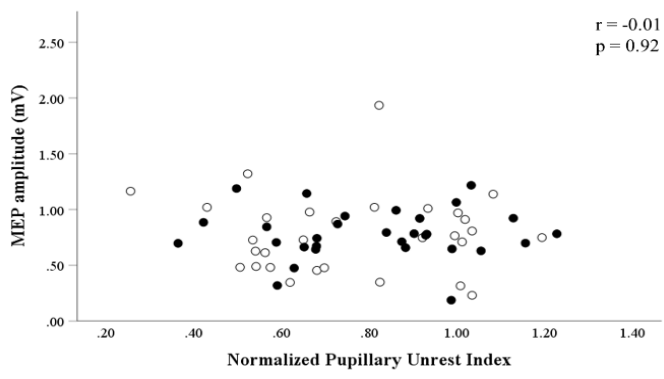

Figure 3.6 Correlations between pupillary unrest index (PUI) and cortical excitability (MEP amplitudes)

A lower PUI value indicates a greater alertness and, thus, a negative correlation between PUI and MEP amplitude indicates a positive effect of alertness on cortical excitability.

a) In $1.0 \mathrm{~mA}$ tACS, placebo; alertness showed a significant positive correlation with cortical excitability.

There were no significant correlations between alertness and cortical excitability in any other session: Sham, placebo (b), 1.0 mA tACS, caffeine (c), or Sham, caffeine (d).

Unfilled circles represent morning sessions, and filled circles represent afternoon sessions. 


\subsubsection{Caffeine consumption behaviours on motor cortex excitability}

The caffeine-naive and the caffeine-adapted groups were compared. RMT was significantly higher in the caffeine-naive group than in the caffeine-adapted groups. A similar pattern was seen for $\mathrm{MT}_{1 \mathrm{mV}}$. MEPPRE did not differ between the groups (See Table 3.2).

Table 3.2 RMT, MT $1 \mathrm{mv}$ and MEPPRE in caffeine-naïve and caffeine-adapted groups

\begin{tabular}{|c|c|c|c|c|c|c|c|c|c|}
\hline & $\begin{array}{c}\text { RMT } \\
\text { (\% MSO) }\end{array}$ & $\mathbf{t}$ & $\mathbf{p}$ & $\begin{array}{c}\mathrm{MT}_{1 \mathrm{mV}} \\
(\% \mathrm{MSO})\end{array}$ & $\mathbf{t}$ & p & $\begin{array}{c}\text { MEPPRE } \\
(\mathrm{mV})\end{array}$ & $\mathbf{t}$ & $\mathbf{p}$ \\
\hline $\begin{array}{l}\text { 1) Caffeine- } \\
\text { naïve }\end{array}$ & $\begin{array}{c}41.46 \pm \\
6.48\end{array}$ & 8.48 & $<0.001 *$ & $\begin{array}{c}51.66 \pm \\
10.27\end{array}$ & 6.27 & $<0.001 *$ & $\begin{array}{c}1.07 \pm \\
0.09\end{array}$ & 0.84 & 0.40 \\
\hline $\begin{array}{l}\text { 2) Caffeine- } \\
\text { adapted } \\
\text { consumers }\end{array}$ & $\begin{array}{c}36.84 \pm \\
5.43\end{array}$ & & & $\begin{array}{c}46.11 \pm \\
9.09\end{array}$ & & & $\begin{array}{c}1.06 \pm \\
0.10\end{array}$ & & \\
\hline
\end{tabular}

Data are presented as means $\pm \mathrm{SD} ; * \mathrm{p}<0.05$;

Abbreviations: RMT $=$ resting motor threshold; $\% \mathrm{MSO}=\%$ of maximum stimulator output; $\mathrm{MT}_{1 \mathrm{mV}}=$ motor threshold which produces $1 \mathrm{mV}$ MEP; $\mathrm{MEP}_{\mathrm{PRE}}=$ baseline motor evoked potential; tACS = transcranial alternating current stimulation.

\subsection{DISCUSSION}

One main finding of this study was that caffeine strengthened and prolonged the LTP-like aftereffects of PAS 25 on the post-stimulation MEPs by up to $37.9 \%$ over placebo in caffeinenaïve subjects and also enhanced the MEP response after tACS and after sham stimulation. The latter was not observed in caffeine consumers. Increased alertness during tACS was associated with a greater increase in MEP amplitudes. Furthermore, our finding that light-deprivation reduced MEP amplitudes confirmed the influence of ambient light on motor cortical excitability (Cambieri et al., 2017; Chen and Huang, 2018).

In Experiment 1, caffeine further enhanced the known LTP-like aftereffects after PAS 25. The effects of PAS 25 are specific at those M1 synapses primed by the incoming afferent somatosensory volley (Nitsche et al., 2007). The direction and magnitude of plasticity after PAS 25 are thought to be modulated by $\mathrm{Ca}^{2+}$ influx into postsynaptic neurons. Drugs such as 
dextromethorphan (DXM) and nimodipine (NDP) as a L-type voltage-gated calcium channel (VGCC) blocker abolished the facilitatory effects of PAS 25, and its effect is reversed into inhibition by T-type VGCC blocker ethosuximide (ESM) (Weise et al., 2017). This finding is in line with the concept that changes in calcium dynamics can modulate the direction of cortical plasticity, in which small calcium increases lead to LTD-like aftereffects, while substantial increases induce LTP-like aftereffects (Grundey et al., 2018). In humans, A1Rs are located mainly at somatodendritic (postsynaptic) and axon terminals (presynaptic) which are the primary targets for caffeine. Competitive binding of caffeine to adenosine receptors inactivate the A1R and interferes with the inhibitory effects of adenosine on transmission at excitatory synapses (Kerkhofs et al., 2018). The results also showed that caffeine acted postsynaptically since moderate caffeine concentrations do not affect glutamatergic synaptic transmission at the presynaptic A1Rs. Caffeine and theophylline are both xanthine derivatives, and theophylline has an inhibitory effect on GABAergic neurons and reduces short intracortical inhibition (SICI) (Nardone et al., 2004). In animal brain slices, caffeine acts presynaptically by inhibiting GABA release and suppressing GABAergic inhibitory postsynaptic currents (IPSCs), which leads to an increase in intracellular calcium (Isokawa, 2016). As discussed above, $\mathrm{Ca}^{2+}$ modulates plasticity aftereffects, and intracellular calcium levels determine whether the effect is LTP or LTD (Lisman, 2001). In this study, the greater increase in intracellular $\mathrm{Ca}^{2+}$ was a result of the PAS 25 effects. In the presence of caffeine, cortical excitability was enhanced and maintained for a longer period of time than the effect with PAS 25 alone. The dynamic change of $\mathrm{Ca}^{2+}$ in shaping synaptic efficacy was also explained using calcium-based plasticity model as described by Graupner \& Brunel (Graupner and Brunel, 2012). This model allows one to predict the plasticity outcomes in different stimulation protocols, and any changes occurring at the synapses modulate the strength of synaptic transmission and firing rates of the neurons. This confirmed the hypothesis that PAS 25 induces plasticity at the specific synaptic connections as described by (Nitsche et al., 2007). We postulate that changes in calcium dynamics in the presence of caffeine increase the firing rate. As a result, we inferred that synaptic transmission was further strengthened by PAS 25 as a known LTP-like plasticity protocol from increases in MEPs.

Similarly, we observed a caffeine-induced increase in cortical excitability under all tACS conditions, although this increase was only significant with $1.0 \mathrm{~mA}$ tACS due to the greater 
variability with $0.4 \mathrm{~mA}$ tACS and Sham stimulation. We were surprisingly unable to reproduce the excitability increase after tACS (1.0 mA) as shown in previous studies (Moliadze, Antal and Paulus, 2010; Moliadze et al., 2012; Zulkifly, Merkohitaj and Paulus, 2020), and thus from Experiment 1 we found no evidence supporting the claim that tACS induced an increase in plasticity in caffeine-naïve participants. We cross-checked all possible explanations for this nonreproducibility including recalibrating the stimulator. The most likely reason is our longer and more fatiguing experimental design, which requires the participants to sit for 2.5 hours with only minimal diversion. Huang and colleagues (Huang et al., 2017) addressed the importance of controlling physical activity before any stimulation protocol, as this has been widely reported to influence plasticity aftereffects. We thus aimed at standardising the hand posture and muscle states, since MEPs are known to vary according to hand posture during motor imagery (Vargas et al., 2004), muscle activation and pattern of activation (i.e. tonic or phasic) (Huang, 2016; Shirota et $a l ., 2017)$. We also restricted the movement of the participant's hand, particularly of the target muscle, to minimise any potential net change in MEP amplitude. As a result, some participants reported that their hand had "gone to sleep" during and after the session. Muscle fatigue induced by a force contraction task has been reported to result in a reduction of cortical excitability in such a manner that the MEP amplitudes were diminished (Lazarski, Ridding and Miles, 2002). In an experiment of similar duration, MEP amplitude was shown to decrease in a control task, in which the participants were required to watch a two-hour documentary on the computer (Solianik et al., 2018). One possible explanation for the changes in cortical excitability in fatigue and during longer experimental protocols is that mental fluctuations and tiredness may increase the alpha power. Changes in oscillatory power are associated with cortical excitability, as lower alpha amplitudes are associated with stronger MEPs (Zarkowski et al., 2006; Sauseng et al., 2009). In the visual system, a lower alpha power at rest indicated a greater cortical excitability as shown by a lowered phosphene threshold (Romei, Brodbeck, et al., 2008; Romei, Rihs, et al., 2008). This indicates that the brain state is an important factor in shaping the direction of plasticity aftereffects, since reducing attention increases the endogenous alpha power. Whenever alpha power reached a ceiling level, which in this case a reduction in attention level, it was no longer possible to modulate the endogenous alpha power and its null effect was compatible with the failure to observe the aftereffects of tACS (Neuling, Rach and Herrmann, 2013). This explanation remains speculative as no EEG was recorded to quantify alpha oscillatory power in this study. However, the alertness 
score is consistent with this possibility since the participants reported being less awake in the placebo sessions with 0.4 mA tACS, Sham and PAS 25.

In Experiment 2, we observed a consistent reduction in cortical excitability, which was most likely caused by the stimulation being carried out with the participants in total darkness. This was a necessary condition for obtaining a reliable reading from the pupillometer. In the visual system, a state of limited visual input reduces the phosphene threshold (PT) indicating increased visual cortex excitability (Boroojerdi et al., 2000; Fierro et al., 2005; Pitskel et al., 2007; de Graaf et al., 2017). This increase persisted for 120 minutes after re-exposure to light, and it has been corroborated by neuroimaging which showed that the increase in visual cortex activation persisted for 30 minutes after re-exposure to light (Boroojerdi et al., 2000). In addition, the facilitatory effects of $10 \mathrm{~Hz}$ rTMS were abolished after $60 \mathrm{~min}$ of light deprivation, and the return of the phosphene threshold to baseline after re-exposure to light was more rapid than after $1 \mathrm{~Hz}$ rTMS (Fierro et al., 2005). However, in the motor cortex light deprivation (i.e. eyes closed state) led to lower excitability as shown by a shallower stimulus-response curve (Chen and Huang, 2018). We found a decrease in MEP amplitudes after a short-term light deprivation that confirmed the results of Cambieri and colleagues (Cambieri et al., 2017) who had demonstrated that the facilitatory effects of rTMS were reduced during 30 minutes in darkness. It seems that the MEP inhibition during darkness remains after re-exposure to light as shown in our study. There was a discrepancy in a study by Leon-Sarmiento and colleagues (Leon-Sarmiento, Bara-Jimenez and Wassermann, 2005) who reported an increase in motor cortex excitability during a 30-minute blindfolding. The MEPs were demonstrated to be higher with eyes closed than with eyes open, and in the dark the excitability tended to be greater than in the light. There is a link between the visual and the motor systems; it was systematically studied using paired pulse TMS which showed that an increase of SICI in M1 after administering a conditioning stimulus (CS) over the visual cortex reflected an inhibitory influence from the visual to the motor cortex (Strigaro et al., 2015). Our participants wore goggles that completely blocked out ambient light. Their eyes were open for ten minutes while they fixated a green dot in the pupillometer. A state of limited visual input is thought to modulate visual alpha power and peak frequency (Webster and Ro, 2020). In the EEG, the increase in alpha amplitudes in conditions without visual input, such as in eyes closed, has been described as the Berger effect (Kirschfeld, 2005; de Graaf et al., 2017). EEG oscillations at certain brain 
rhythms (alpha and beta) were correlated with the excitability in the motor cortex. EEG alpha activity is related to motor cortex excitability with higher cortical activation being associated with a lower amplitude of alpha oscillations (Sauseng et al., 2009).

Unexpectedly, the facilitatory effect of caffeine, which was demonstrated in Experiment 1, was reversed to inhibition. We attribute this suppression of cortical excitability to the ten minutes of light deprivation. Post MEP inhibition was observed for 30 minutes under all conditions including the control condition, i.e. sham stimulation, morning, placebo. This emphasises that the initial brain-state modulates the brain stimulation outcomes. On the one hand, we may explain this finding by addressing neuronal adaptation phenomena. Silvanto and colleagues addressed the issue of adaptation and priming in shaping the excitation and inhibition of the stimulated regions and also the behaviour outcomes (Silvanto, Muggleton and Walsh, 2008). We need to assume that adaptation to darkness modulates neuronal excitability in the motor cortex. This argument is in line with a study by Leon-Sarmiento and colleagues (Leon-Sarmiento, Bara-Jimenez and Wassermann, 2005) which claimed that cortical excitability increased in darkness. Here we found MEP inhibition in a short period of light deprivation, whereas in previous studies cortical excitability was increased in the visual and motor cortex after long period of light deprivation (45 minutes) (Boroojerdi et al., 2000; Fierro et al., 2005; Leon-Sarmiento, Bara-Jimenez and Wassermann, 2005; Pitskel et al., 2007; de Graaf et al., 2017). Another possible explanation for MEP inhibition in our study is the nature of pupillometer measurement in which the participants were instructed to focus their attention on a green dot. Shifting the focus of attention modulates intracortical inhibitory circuit in M1. In a study by Kuhn and colleagues (Kuhn et al., 2017), externally focused attention (i.e. control and concentrate on the position of the goniometer) adopted during the motor task change inhibitory activity within M1 compared with internally focused attention (i.e. contract and concentrate on finger muscles).

In contrast to Experiment 1, we found that $1.0 \mathrm{~mA}$ tACS induced excitatory MEPs increase compared with Sham. This confirmed the excitatory effects of this protocol, as shown by previous studies (Moliadze, Antal and Paulus, 2010; Moliadze et al., 2012; Zulkifly, Merkohitaj and Paulus, 2020). We attributed the difference between the findings of the two experiments to the difference in the studied groups, namely caffeine-naïve participants in Experiment 1 and caffeine-adapted 
consumers in Experiment 2. Chronic caffeine consumption is associated with modulation of A1R expression. In female rats, treatment with caffeine and theophylline was reported to downregulate the A1R expression (León et al., 2002). In contrast to male rats, LTP decreased by caffeine consumption was attributed to upregulation of the A1R (Blaise et al., 2018). As it remains controversial in which direction the expression of A1R modulates the LTP-like effect, we can only speculate that these conflicting results in the two experiments are due to A1R expression.

As expected, we found that MEPs were associated with an increase in alertness during tACS. This result confirmed the findings of Stefan and colleagues (Stefan, Wycislo and Classen, 2004), who claimed that attention modulated the LTP-like aftereffects of PAS. These neurophysiological effects can be explained by an increase in the firing rates as a result of an increase in gamma frequency and reduced low-frequency synchronization of the neurons (Fries, 2001). However, this finding is inconsistent with a hypothesis of Paulus and Rothwell (Paulus and Rothwell, 2016), who believe that attention activates neurons and thereby increases the opening of ion channels concomitant with increases in membrane conductance. As transmembrane resistance is reduced, the degree of polarization of the neurons will be reduced resulting in smaller aftereffects of tACS. However, synapse specific plasticity, such as induced by PAS, must be differentiated from unspecific plasticity such as that induced by tACS.

We showed that the effects of caffeine on alertness and daytime sleepiness could be quantified by pupillometry. Pupillary oscillations were more stable and less fluctuating after caffeine administration. The pupil diameters found in this study that ranged from $6.7 \pm 1.2$ to 7.0 $\pm 1.3 \mathrm{~mm}$ compared very well with those described in the literature. Daguet and colleagues (Daguet, Bouhassira and Gronfier, 2019) reported a raw pupil diameter in the range of 5.8 to 8.7 $\mathrm{mm}($ mean $=7.4 \pm 0.7 \mathrm{~mm})$, and a mean daytime pupil diameter of $7.24 \pm 0.40 \mathrm{~mm}$ (Wilhelm et al., 2001). The pupillary unrest index (PUI) was also in the range of previous studies (Wilhelm et al., 2001; Daguet, Bouhassira and Gronfier, 2019). Smaller deviations can be caused by the nonstimulating study design compared with a study by Wilhem and colleagues (Wilhelm et al., 2001) since the participants in their study performed some tasks such as reading, playing games or listening to music. Subjective factors might contribute to this deviation, as all of the participants in this experiment were caffeine users. Caffeine-abstention on an experiment day might lead to 
withdrawal symptoms, such as drowsiness, fatigue, low motivation with a concomitantly higher PUI.

The two studied groups differed in their consumption behaviour; caffeine-naïve subjects participated in Experiment 1 and caffeine consumers in Experiment 2. We found evidence of a higher excitability in caffeine consumers as shown by a lower motor threshold (RMT and MT $1 \mathrm{mV})$. Both are direct indicators of cortical facilitation, and standard measures in drug studies (refer to

review by Ziemann and colleagues (Ziemann et al., 2015) and Caipa and colleagues (Caipa, Alomar and Bashir, 2018)). No significant effect on the motor threshold (RMT and AMT) was seen after oral administration of $200 \mathrm{mg}$ theophylline, another adenosine receptor antagonist (Nardone et al., 2004).

The present study does have its limitations. The main aim was to examine the effects of caffeine on the chosen LTP/LTD-like plasticity protocols. More parameters can be controlled in future studies. First, there is a large variability in the response to caffeine due to genetic polymorphism and activity of the metabolising enzyme CYP1A2 (Sachse et al., 1999; Mejia and Ramirez-Mares, 2014; Matthaei et al., 2016). The administered caffeine dose would have had to be adjusted to take the individual's pharmacokinetics and pharmacodynamics into account. Second, MEPs have been established as an effective tool for quantifying drug effects on the human brain (Nitsche et al., 2012). Directly measuring brain activity in the EEG, e.g. with transcranial evoked potentials (TEP) could extend cortical excitability measures beyond the motor cortex and, when combined with fMRI, could reveal information on attentional networks (Ozdemir et al., 2020).

\subsection{CONCLUSIONS}

Caffeine enhanced the synapse-specific LTP-like effects of PAS 25. MEPs increased after caffeine administration in the PAS 25 protocol. As in caffeine-naïve participants, MEPs were generally increased after caffeine ingestion, but there was no evidence that tACS induced facilitatory effects. The facilitatory effect of tACS was only observed in in the caffeine consumers in Experiment 2. Plasticity induction was not influenced by time of day. In addition, limited visual input during 
stimulation reduced motor cortex excitability, and there was a correlation between alertness during tACS and motor cortex excitability. From these findings we conclude, first, that caffeine intake should be avoided for an extended period prior to non-invasive brain stimulation techniques and plasticity induction studies to ensure optimal data reproducibility. Second, in methodological aspects, the brain states during plasticity studies of the motor cortex such as light exposure and subjective alertness should be documented and controlled to ensure that any excitability changes such as stimulation of non-motor areas do not confound plasticity induction in the motor cortex.

\section{Funding}

This work was supported by the Ministry of Education (MOE), Malaysia

\section{Conflict of Interest Statement}

None of the authors have potential conflicts of interest to be disclosed

\section{Acknowledgements}

We would like to thank Prof. Thomas Crozier for proofreading, Dr. Islam Halawa for comments on an early version of the manuscript and Prof. Michael Nitsche for statistical support. 
Supplementary Table 3.1 Experiment 1: Motor thresholds, baseline MEP and ANOVAs

a) RMT, MT $1 \mathrm{mv}$ and baseline MEP (MEPPRE)

\begin{tabular}{llll}
\hline & RMT $(\%$ MSO) & MT 1 imv $(\%$ MSO) & MEPPRe $(\mathbf{m V})$ \\
\hline 1) tACS (1.0 mA) & & & \\
placebo & $42.0 \pm 6.9$ & $52.4 \pm 11$ & $1.06 \pm 0.10$ \\
caffeine & $41.2 \pm 6.8$ & $52.1 \pm 11$ & $1.07 \pm 0.09$ \\
2) tACS (0.4 mA) & & & \\
placebo & $41.3 \pm 5.8$ & $51.6 \pm 10$ & $1.09 \pm 0.08$ \\
caffeine & $41.4 \pm 6.9$ & $51.2 \pm 11$ & $1.04 \pm 0.08$ \\
3) tACS (Sham) & & & \\
placebo & $41.2 \pm 5.7$ & $50.9 \pm 10$ & $1.08 \pm 0.11$ \\
caffeine & $41.6 \pm 6.3$ & $51.5 \pm 10$ & $1.08 \pm 0.09$ \\
4 ) PAS 25 & & & \\
placebo & $41.8 \pm 6.5$ & $52.2 \pm 11$ & $1.06 \pm 0.10$ \\
caffeine & $41.1 \pm 7.5$ & $51.3 \pm 10$ & $1.08 \pm 0.10$ \\
\hline
\end{tabular}

b) Results of ANOVAs

\begin{tabular}{llllll}
\hline & Parameters & d.f. & $\mathbf{F}$ & $\mathbf{\eta}_{\mathbf{p}}{ }^{2}$ & $\mathbf{p}$ \\
\hline 1) RMT & Stimulation & 3,87 & 0.22 & 0.01 & 0.882 \\
& Drug & 1,29 & 0.38 & 0.01 & 0.544 \\
& Stimulation x Drug & 3,87 & 1.18 & 0.04 & 0.324 \\
& & & & \\
$\mathrm{MT}_{1 \mathrm{mV}}$ & Stimulation & 3,87 & 1.48 & 0.05 & 0.226 \\
& Drug & 1,29 & 0.15 & 0.01 & 0.698 \\
& Stimulation x Drug & $2.12,61.36$ & 0.60 & 0.02 & 0.563 \\
3EP $\mathrm{MRE}_{\mathrm{PRE}}$ & & & & \\
& Stimulation & 3,87 & 0.10 & 0.00 & 0.959 \\
& Drug & 1,29 & 0.41 & 0.01 & 0.529 \\
& Stimulation x Drug & 3,87 & 1.38 & 0.05 & 0.255 \\
\hline
\end{tabular}

Data are presented as mean \pm SD and $*$ indicates $\mathrm{p}<0.05$. Abbreviations: RMT $=$ resting motor threshold; \% MSO $=\%$ of maximum stimulator output; $\mathrm{MT}_{1 \mathrm{mV}}=$ motor threshold which produces $1 \mathrm{mV}$ MEP; $\mathrm{MEP}_{\mathrm{PRE}}=$ baseline motor evoked potential; PAS = paired associative stimulation; tACS = transcranial alternating current stimulation.

Supplementary Table 3.2 Experiment 2: Motor thresholds, baseline MEP and ANOVAs

a) RMT, MT1mV and baseline MEP (MEPPRE)

RMT (\% MSO) MT1mv (\% MSO) MEPpRE (mV)

1) tACS (1.0 mA); afternoon

placebo

$36.4 \pm 5.4$

$46.0 \pm 9.4$

$1.11 \pm 0.09$

caffeine

$37.0 \pm 5.7$

$45.9 \pm 8.5$

$1.07 \pm 0.13$

2) tACS (1.0 mA); morning 


\begin{tabular}{lccc}
\hline placebo & $36.8 \pm 5.3$ & $45.9 \pm 9.2$ & $1.06 \pm 0.11$ \\
caffeine & $37.2 \pm 4.8$ & $46.6 \pm 9.0$ & $1.04 \pm 0.08$ \\
3) tACS (Sham);afternoon & & & \\
placebo & $36.9 \pm 5.7$ & $46.4 \pm 9.5$ & $1.08 \pm 0.09$ \\
caffeine & $36.8 \pm 5.5$ & $46.2 \pm 9.5$ & $1.05 \pm 0.10$ \\
4) tACS (Sham); morning & & & \\
placebo & $36.6 \pm 6.0$ & $45.12 \pm 9.2$ & $1.05 \pm 0.11$ \\
caffeine & $37.0 \pm 5.6$ & $46.8 \pm 9.3$ & $1.05 \pm 0.09$ \\
\hline
\end{tabular}

b) Results of ANOVAs

\begin{tabular}{|c|c|c|c|c|c|}
\hline & Parameters & d.f. & $\mathbf{F}$ & $\eta_{p}^{2}$ & $\mathbf{p}$ \\
\hline \multirow[t]{7}{*}{ 1) RMT } & Stimulation & 1,29 & 0.00 & 0.00 & 0.989 \\
\hline & Day & 1,29 & 0.03 & 0.00 & 0.877 \\
\hline & Drug & 1,29 & 0.25 & 0.01 & 0.619 \\
\hline & Stimulation x Day & 1,29 & 0.06 & 0.00 & 0.810 \\
\hline & Stimulation x Drug & 1,29 & 0.05 & 0.00 & 0.820 \\
\hline & Day x Drug & 1,29 & 0.01 & 0.00 & 0.940 \\
\hline & Stimulation x Day x Drug & 1,29 & 0.08 & 0.00 & 0.781 \\
\hline \multicolumn{6}{|c|}{ 2) $\mathrm{MT}_{1 \mathrm{mV}}$} \\
\hline & Stimulation & 1,29 & 0.00 & 0.00 & 0.979 \\
\hline & Day & 1,29 & 0.00 & 0.00 & 0.987 \\
\hline & Drug & 1,29 & 0.20 & 0.01 & 0.660 \\
\hline & Stimulation x Day & 1,29 & 0.07 & 0.00 & 0.800 \\
\hline & Stimulation x Drug & 1,29 & 0.04 & 0.00 & 0.848 \\
\hline & Day x Drug & 1,29 & 0.40 & 0.01 & 0.532 \\
\hline & Stimulation x Day x Drug & 1,29 & 0.05 & 0.00 & 0.829 \\
\hline \multicolumn{6}{|c|}{ 3) $\mathrm{MEP}_{\mathrm{PRE}}$} \\
\hline & Stimulation & 1,29 & 2.27 & 0.07 & 0.142 \\
\hline & Day & 1,29 & 4.57 & 0.14 & $0.041 *$ \\
\hline & Drug & 1,29 & 2.01 & 0.07 & 0.167 \\
\hline & Stimulation x Day & 1,29 & 1.33 & 0.04 & 0.257 \\
\hline & Stimulation $\mathrm{x}$ Drug & 1,29 & 0.10 & 0.00 & 0.756 \\
\hline & Day x Drug & 1,29 & 1.72 & 0.06 & 0.200 \\
\hline & Stimulation x Day x Drug & 1,29 & 0.00 & 0.00 & 0.985 \\
\hline
\end{tabular}

Data are presented as mean \pm SD and $*$ indicates $\mathrm{p}<0.05$. Abbreviations: RMT $=$ resting motor threshold; \% MSO $=\%$ of maximum stimulator output; $\mathrm{MT}_{1 \mathrm{mV}}=$ motor threshold which produces $1 \mathrm{mV}$ MEP; $\mathrm{MEP}_{\mathrm{PRE}}=$ baseline motor evoked potential; tACS $=$ transcranial alternating current stimulation.

\section{Supplementary Table 3.3 Number of participants reporting sensations during tACS, and their ratings of} stimulation type in Experiment 1

a) tACS sensation perception

\begin{tabular}{lcc}
\hline & Yes, $\mathbf{n}(\boldsymbol{\%})$ & No, $\mathbf{n}(\boldsymbol{\%})$ \\
\hline $\begin{array}{l}\text { 1) tACS }(1.0 \mathrm{~mA}) \\
\text { placebo }\end{array}$ & $15(19.2)$ & $15(14.7)$ \\
\hline
\end{tabular}




\begin{tabular}{lcc}
\hline caffeine & $17(21.8)$ & $13(12.7)$ \\
$2)$ tACS $(0.4 \mathrm{~mA})$ & & \\
placebo & $6(7.7)$ & $24(23.5)$ \\
caffeine & $6(7.7)$ & $24(23.5)$ \\
$3)$ tACS (Sham) & & \\
placebo & $18(23.1)$ & $12(11.8)$ \\
caffeine & $16(20.5)$ & $14(13.7)$ \\
\hline
\end{tabular}

b) Rating of stimulation type

\begin{tabular}{lccc}
\hline & $\begin{array}{c}\text { True stimulation, } \\
\mathbf{n}(\boldsymbol{\%})\end{array}$ & $\begin{array}{c}\text { Placebo stimulation, } \\
\mathbf{n}(\boldsymbol{\%})\end{array}$ & $\begin{array}{c}\text { Don't know, } \\
\mathbf{n}(\boldsymbol{\%})\end{array}$ \\
\hline $\begin{array}{l}\text { 1) tACS }(1.0 \mathrm{~mA}) \\
\text { placebo }\end{array}$ & $12(20.3)$ & & \\
caffeine & $13(22.0)$ & $9(11.9)$ & $10(18.5)$ \\
$\begin{array}{l}\text { 2) tACS }(0.4 \mathrm{~mA}) \\
\text { placebo }\end{array}$ & & & $8(14.4)$ \\
caffeine & $4(6.8)$ & $17(25.4)$ & \\
3) tACS (Sham) & $10(16.9)$ & $10(14.9)$ & $10(16.7)$ \\
placebo & & & \\
caffeine & $10(16.9)$ & $12(17.9)$ & $8(14.8)$ \\
\hline
\end{tabular}

Supplementary Table 3.4 Number of participants reporting sensations during tACS, and their ratings of stimulation quality in Experiment 2

a) tACS sensation perception

\begin{tabular}{lll}
\hline & Yes, $\mathbf{n}(\boldsymbol{\%})$ & No, $\mathbf{n}(\boldsymbol{\%})$ \\
\hline 1) tACS (1.0 mA) & & \\
afternoon, placebo & $13(13.3)$ & $16(11.9)$ \\
afternoon, caffeine & $8(8.2)$ & $21(15.6)$ \\
morning, placebo & $13(13.3)$ & $17(12.6)$ \\
morning, caffeine & $15(15.3)$ & $14(10.4)$ \\
2) tACS (Sham) & & \\
afternoon, placebo & $11(11.2)$ & $19(14.1)$ \\
afternoon, caffeine & $12(12.2)$ & $16(11.9)$ \\
morning, placebo & $15(15.3)$ & $15(11.1)$ \\
morning, caffeine & $11(11.2)$ & $17(12.6)$ \\
\hline
\end{tabular}

b) Rating of stimulation type

\begin{tabular}{lccc}
\hline & $\begin{array}{c}\text { True stimulation, } \\
\mathbf{n}(\%)\end{array}$ & $\begin{array}{c}\text { Placebo stimulation, } \\
\mathbf{n}(\%)\end{array}$ & $\begin{array}{c}\text { Don't know, } \\
\mathbf{n}(\%)\end{array}$ \\
\hline $\begin{array}{l}\text { 1) tACS }(1.0 \mathrm{~mA}) \\
\text { afternoon, placebo }\end{array}$ & $10(10.5)$ & & \\
\hline
\end{tabular}




\begin{tabular}{lccc}
\hline afternoon, caffeine & $8(8.4)$ & $9(12.7)$ & $12(17.6)$ \\
morning, placebo & $12(12.6)$ & $9(12.7)$ & $9(13.2)$ \\
morning, caffeine & $13(13.7)$ & $7(9.9)$ & $9(13.2)$ \\
2) tACS (Sham) & & & \\
afternoon, placebo & $13(13.7)$ & $11(15.5)$ & $6(8.8)$ \\
afternoon, caffeine & $8(8.4)$ & $11(15.5)$ & $9(13.3)$ \\
morning, placebo & $16(16.8)$ & $9(12.7)$ & $5(7.4)$ \\
morning, caffeine & $15(15.8)$ & $7(9.9)$ & $7(10.3)$ \\
\hline
\end{tabular}

Supplementary Table 3.5 Experiment 1: Results of the ANOVAs

\section{Parameters}

1) PAS 25

All data

2) PAS 25

Pooled data

3) tACS

All data

4) tACS

Pooled data

Time
Drug
Time x Drug

Time
Drug
Time x Drug

Time

Stimulation

Drug

Time x Stimulation

Time $\mathrm{x}$ Drug

Stimulation $\mathrm{x}$ Drug

Time x Stimulation x Drug d.f.

$4.71,136.44$

1,29

7, 203

2, 58

1,29

2, 58

$3.08,89.38$

2, 58

1,29

$7.01,203.34$

7, 203

2, 58

$8.22,238.39$
F

6.86
9.84
1.89

0.19

0.25

0.06

0.45

0.25

0.17

5.82

24.03

5.86

1.74

0.06

0.01

2, 58

2, 58

1,29

4, 116

2, 58

2, 58

4,116
0.12

0.04

0.05

0.02

0.04

1.05

4.12

1.11

1.49

0.72

(1)

0.10

0.01

0.11

0.01

0.12

0.03

0.02

3.76

0.24

0.96

0.71 p
$<0.001 *$
$0.004^{*}$
0.072

$<0.001 *$

$0.005^{*}$

$0.005^{*}$

0.163

0.819

0.050 *

0.358

0.173

0.493

0.403

Stimulation $x$ Drug

0.072

0.786

Time x Stimulation x Drug

0.067

0.862

$0.040^{*}$

0.389

0.540

$*$ indicates $\mathrm{p}<0.05$. Abbreviations: d.f. $=$ degree of freedom; $\eta_{\mathrm{p}}{ }^{2}=$ partial eta square; $\mathrm{PAS}=$ paired associative stimulation; tACS $=$ transcranial alternating current stimulation. 


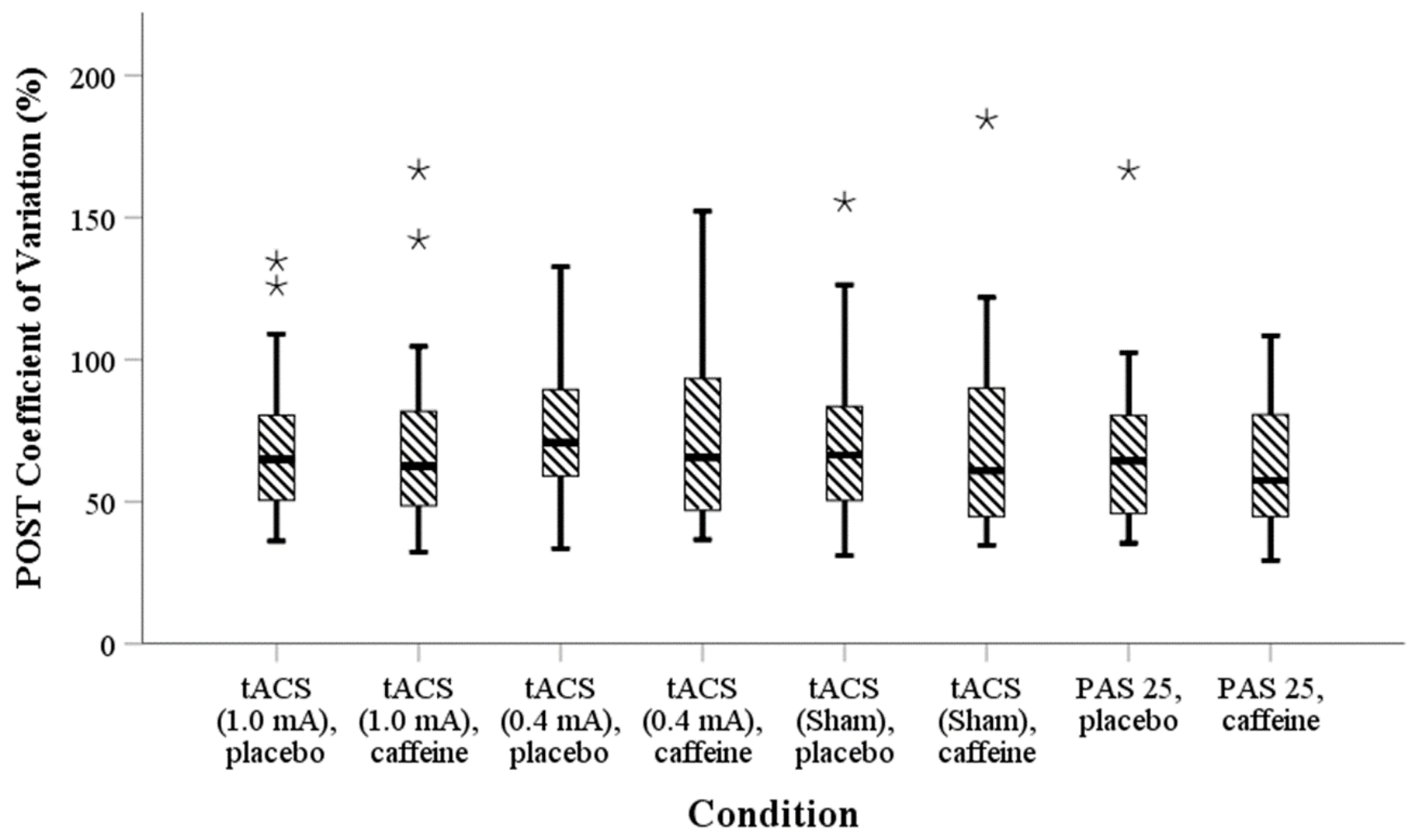

Supplementary Figure 3.1 The distribution of POST MEP recordings represented by coefficient of variation $(\mathrm{CV})$.

The box represents upper and lower quartiles and median and whiskers indicate the minimum and maximum observations. ${ }^{*}$ indicates outliers in the respective sessions.

Supplementary Table 3.6 Experiment 2: Results of ANOVAs (all data)

\begin{tabular}{lllll}
\hline Parameters & d.f. & $\mathbf{F}$ & $\mathbf{n}_{\mathbf{p}}^{\mathbf{2}}$ & $\mathbf{p}$ \\
\hline Time & $5.02,145.50$ & 12.42 & 0.30 & $<0.001^{*}$ \\
Stimulation & 1,29 & 10.45 & 0.27 & $0.003^{*}$ \\
Day & 1,29 & 0.13 & 0.00 & 0.727 \\
Drug & 1,29 & 0.96 & 0.03 & 0.335 \\
Time x Stimulation & 7,203 & 1.41 & 0.05 & 0.203 \\
Time x Day & 7,203 & 1.29 & 0.04 & 0.259 \\
Stimulation x Day & 1,29 & 2.63 & 0.08 & 0.116 \\
Time x Stimulation x Day & $5.67,164.33$ & 2.45 & 0.08 & $0.030^{*}$ \\
Time x Drug & 7,203 & 1.07 & 0.04 & 0.383 \\
Stimulation x Drug & 1,29 & 0.16 & 0.01 & 0.696 \\
Time x Stimulation x Drug & $5.15,149.40$ & 0.48 & 0.02 & 0.795 \\
Day x Drug & 1,29 & 0.08 & 0.00 & 0.781 \\
Time x Day x Drug & $4.87,141.30$ & 0.75 & 0.03 & 0.583 \\
\hline
\end{tabular}




\begin{tabular}{lllll}
\hline Stimulation x Day x Drug & 1,29 & 2.40 & 0.08 & 0.133 \\
Time x Stimulation x Day x Drug & 7,203 & 1.36 & 0.05 & 0.223 \\
\hline
\end{tabular}

$*$ indicates $\mathrm{p}<0.05$. Abbreviations: d.f. $=$ degree of freedom; $\eta_{\mathrm{p}}{ }^{2}=$ partial eta square.

Supplementary Table 3.7 Experiment 2: Results of ANOVAs (pooled data)

\begin{tabular}{lllll}
\hline Parameters & d.f. & $\mathbf{F}$ & $\mathbf{\eta}_{\mathbf{p}}^{\mathbf{2}}$ & $\mathbf{p}$ \\
\hline Time & 2,58 & 31.74 & 0.52 & $<0.001^{*}$ \\
Stimulation & 1,29 & 11.18 & 0.28 & $0.002^{*}$ \\
Day & 1,29 & 0.03 & 0.00 & 0.876 \\
Drug & 1,29 & 0.57 & 0.02 & 0.457 \\
Time x Stimulation & 2,58 & 3.70 & 0.11 & $0.031^{*}$ \\
Time x Day & $1.57,45.48$ & 0.54 & 0.02 & 0.546 \\
Stimulation x Day & 1,29 & 1.28 & 0.04 & 0.268 \\
Time x Stimulation x Day & 2,58 & 2.55 & 0.08 & 0.087 \\
Time x Drug & $1.42,41.27$ & 0.45 & 0.02 & 0.574 \\
Stimulation x Drug & 1,29 & 1.06 & 0.04 & 0.312 \\
Time x Stimulation x Drug & 2,58 & 1.89 & 0.06 & 0.161 \\
Day x Drug & 1,29 & 1.06 & 0.04 & 0.313 \\
Time x Day x Drug & 2,58 & 1.86 & 0.06 & 0.165 \\
Stimulation x Day x Drug & 1,29 & 4.37 & 0.13 & $0.045^{*}$ \\
Time x Stimulation x Day x Drug & 2,58 & 2.63 & 0.08 & 0.081 \\
\hline
\end{tabular}

$*$ indicates $\mathrm{p}<0.05$. Abbreviations: d.f. $=$ degree of freedom; $\Pi_{\mathrm{p}}{ }^{2}=$ partial eta square.

Supplementary Table 3.8 Pupillary unrest index (PUI) and pupil diameter (PD)

\begin{tabular}{|c|c|c|c|c|c|c|}
\hline & PUI & $t$ & $\mathbf{p}$ & PD & $t$ & $\mathbf{p}$ \\
\hline \multicolumn{7}{|c|}{$\begin{array}{l}\text { 1) tACS } \\
\text { (1.0 mA); } \\
\text { afternoon }\end{array}$} \\
\hline placebo & $9.07 \pm 4.15$ & 3.85 & $0.001 *$ & $6.66 \pm 1.19$ & -1.89 & 0.07 \\
\hline caffeine & $6.57 \pm 3.54$ & & & $7.01 \pm 1.28$ & & \\
\hline \multicolumn{7}{|c|}{$\begin{array}{l}\text { 2) tACS } \\
(1.0 \mathrm{~mA}) \text {; } \\
\text { morning }\end{array}$} \\
\hline placebo & $8.25 \pm 3.83$ & 2.72 & $0.01 *$ & $6.89 \pm 1.25$ & -1.79 & 0.09 \\
\hline caffeine & $6.34 \pm 2.89$ & & & $7.10 \pm 1.08$ & & \\
\hline \multicolumn{7}{|c|}{$\begin{array}{l}\text { 3) tACS (Sham); } \\
\text { afternoon }\end{array}$} \\
\hline placebo & $9.54 \pm 3.86$ & 3.10 & $0.005 *$ & $6.79 \pm 1.08$ & -1.21 & 0.24 \\
\hline caffeine & $7.22 \pm 3.63$ & & & $6.98 \pm 1.33$ & & \\
\hline \multicolumn{7}{|c|}{$\begin{array}{l}\text { 4) tACS (Sham); } \\
\text { morning }\end{array}$} \\
\hline placebo & $8.47 \pm 4.06$ & 2.97 & $0.006 *$ & $7.01 \pm 1.32$ & -0.96 & 0.34 \\
\hline caffeine & $6.59 \pm 3.50$ & & & $7.07 \pm 1.36$ & & \\
\hline
\end{tabular}

Data are presented as mean \pm SD and $*$ indicates $\mathrm{p}<0.05$. Abbreviations: $\mathrm{PUI}=$ pupillary unrest index; PD $=$ pupil diameter; $\mathrm{tACS}=$ transcranial alternating current 


\section{CHAPTER IV}

THE ROLES OF CAFFEINE AND CORTICOSTEROIDS IN MODULATING CORTICAL EXCITABILITY AFTER PAIRED ASSOCIATIVE STIMULATION (PAS) AND TRANSCRANIAL ALTERNATING CURRENT STIMULATION (TACS) IN CAFFEINENAÏVE AND CAFFEINE-ADAPTED SUBJECTS.

\section{Submitted as:}

Mohd Faizal Mohd Zulkifly, Ornela Merkohitaj, Walter Paulus, Jürgen Brockmöller. The roles of caffeine and corticosteroids in modulating cortical excitability after paired associative stimulation (PAS) and transcranial alternating current stimulation (tACS) in caffeine-nä̈ve and caffeine-adapted subjects.

\subsection{ABSTRACT}

The modulatory effects of non-invasive brain stimulation (NIBS) protocols are commonly attributed to changes in $\mathrm{Ca}^{2+}$ dynamics, in the expression of inhibitory or excitatory receptors, and also to the sensitivity of the response of the hypothalamic-pituitary-adrenal (HPA) axis. In this study, we explored the relationship between the concentrations of caffeine, corticosteroids and the excitability and also plasticity of the motor cortex. We also examined the effects of caffeine, stimulation and time of day on the salivary concentrations of the corticosteroids. Sixty participants were recruited for a randomized, controlled, double-blind study. Two experiments were performed with eight sessions each: Experiment 1 - caffeine-naïve participants ( $\mathrm{n}=30)$, and Experiment 2 caffeine consumers $(n=30)$. There were two interventions in each session. In intervention 1 the 
participants were given a tablet containing either $200 \mathrm{mg}$ caffeine or placebo. Intervention 2 was stimulation without (Experiment 1) or with (Experiment 2) adjustment for time of day. We found that higher prestimulation caffeine concentrations were associated with higher baseline cortical excitability in caffeine-adapted participants. Increased poststimulation caffeine concentrations were observed together with decreased motor evoked potential (MEP) amplitudes after Sham in caffeine naïve subjects. However, no relationships between poststimulation caffeine, poststimulation corticosteroid concentrations and plasticity aftereffects was found. Caffeine increased the saliva corticosteroid concentrations in both caffeine-naïve and caffeine-adapted participants. In caffeine consumers, the levels of corticosteroid concentrations were higher in the morning than in the afternoon after caffeine intake. Gender affected the levels of corticosteroids in both groups. PAS and tACS did not elicit changes in the corticosteroid concentrations in either group. We conclude that moderate caffeine consumption changes cortical excitability but does not alter plasticity aftereffects. The latter effects also applied to circulating corticosteroids concentrations.

This study was registered in the ClinicalTrials.gov with these registration IDs:

1) NCT03720665

https://clinicaltrials.gov/ct2/results? cond=NCT03720665\&term $=\&$ cntry $=\&$ state $=\&$ city $=\&$ dist $=$

2) NCT04011670

https:/clinicaltrials.gov/ct2/results? cond=\&term $=$ NCT04011670\&cntry $=\&$ state $=\&$ city $=\&$ dist $=$

Keywords: cortisol, cortisone, motor cortex, transcranial magnetic stimulation (TMS), variability

\subsection{INTRODUCTION}

Brain excitability and plasticity responses to stimulation are affected by various factors including age, genetics, skull thickness, attention, time of day, brain states, medications use, hormone levels and others (Stefan, Wycislo and Classen, 2004; Cheeran et al., 2008; Paulus et al., 2008; Sale, 
Ridding and Nordstrom, 2008; Silvanto, Muggleton and Walsh, 2008; Thirugnanasambandam et al., 2011; Conde et al., 2012b; Kamke et al., 2012; Nitsche et al., 2012; Freitas, Farzan and Pascual-Leone, 2013). In dealing with inter-individual variability, researchers grouped subjects into responders and non-responders according to their motor evoked potentials (MEP) using a cluster analysis (López-Alonso et al., 2014; Wiethoff, Hamada and Rothwell, 2014). Some studies have shown that fewer than half of the participants may respond to brain stimulation. In one study, about $40 \%$ of the participants responded to PAS 25, anodal transcranial direct current stimulation and intermittent theta-burst stimulation (López-Alonso et al., 2014). In other studies, about 25\% responded to continuous theta-burst stimulation (cTBS), i.e. inhibition with cTBS and facilitation with iTBS (Hamada et al., 2013), 52\% responded to PAS (Müller-Dahlhaus et al., 2008), and with tDCS only $36 \%$ showed facilitatory effects with anodal and inhibitory effects with cathodal stimulation as expected (Wiethoff, Hamada and Rothwell, 2014). This difficult to reproduce issue has raised concerns for NIBS as a therapeutic tool and had led to a surge in studies to optimize the effectiveness of the stimulation methods. Here we aimed at identifying other potential sources of scatter in the response to NIBS, which include caffeine intake, time of day, and stress related endocrine changes.

Caffeine is a psychostimulant that acts as a competitive antagonist at the adenosine receptor (Kerkhofs et al., 2018; Calker et al., 2019). Differential effects of caffeine on NIBS have already been reported in animal and human studies. In patients, caffeine impaired the effectiveness of deep brain stimulation (DBS) in treating tremor symptoms (Bekar et al., 2008). In animals, long term depression (LTD)-like effects of cathodal transcranial direct current stimulation were reduced as shown by the greater percentage change in the local field potential (LFP) amplitudes in the presence of the selective A1 adenosine receptor antagonist DPCPX (Marquez-Ruiz et al., 2012). The long term potentiation (LTP)-like effect of iTBS was abolished in rats treated with caffeine (Blaise et al., 2018). A recent study in caffeine-naïve participants showed that the excitatory aftereffects of tACS were reversed into inhibition after ingesting espresso containing caffeine, and its excitatory effects were not observed with decaffeinated espresso (Zulkifly, Merkohitaj and Paulus, 2020). A reduction of the LTP-like effects was also observed with quadripulse transcranial magnetic stimulation (QPS) after administration of $200 \mathrm{mg}$ caffeine (Hanajima et al., 2019). The 
behaviour gain of anodal tDCS did not improve after caffeine as shown by the lack of effects on muscle strength (Lattari et al., 2019).

The effects of caffeine vary widely between subjects. Even after intake of exactly the same dose of caffeine there is a more than tenfold variation in systemic (blood and tissue) concentrations of caffeine due to environmental and genetic factors (Sachse et al., 1999; Matthaei et al., 2016). In addition, a common experience is that volunteers often do not report exactly their caffeine use, sometimes are not aware about their intake of caffeine-containing beverages, or do not entirely follow study instructions concerning caffeine intake. To control for all that variation, concentrations of caffeine may be measured in blood or saliva. Concentrations in saliva correlate well with blood concentrations (Scott, Chakraborty and Marks, 1984), but taking saliva is less invasive and irritating compared with blood sampling.

Salivary cortisol and cortisone concentrations resemble those of unbound cortisol in plasma. Unbound (free) cortisol is the biologically active form of the hormone. Salivary cortisone is formed from cortisol by the 11 beta-hydroxysteroid dehydrogenase when passing the salivary glands. In saliva, cortisone may better represent total plasma cortisol and stress than salivary cortisol itself (Perogamvros et al., 2010; Mezzullo et al., 2016; Bae et al., 2019). Cortisol is not only involved in the stress response but also in brain functions such as arousal, sleep-wake regulation, motor skill learning, and memory retrieval (Oster et al., 2017). In rodent studies, high serum or hippocampal corticosterone (the rodent analogy of human cortisol) concentrations were associated with the lower amplitude of LTP (Bennett et al., 1991; Pavlides, Watanabe and McEwen, 1993), impaired memory performance (de Quervain et al., 2000; Wolf, 2009; Hakamata et al., 2019) and reduced perceptual learning (Dinse et al., 2017). TMS is perceived as a nonstressful stimulus, it interacts with hypothalamic-pituitary-adrenal axis as shown by a reduction in corticosterone concentrations in male rats six and 24 hours after a single session of TMS (Hedges et al., 2002). HF-rTMS of the left prefrontal cortex of depressive patients led via the hypothalamic-pituitary-adrenal axis to a reduction in salivary cortisol (Baeken, R. De Raedt, et al., 2009). In healthy humans, cathodal tDCS induced a cortisol increase, while anodal tDCS reduced the salivary cortisol response to the stress test (Antal et al., 2014). A further reduction of cortisol levels after anodal tDCS was reported in combination to exposure to images with a negative 
emotional content (Brunoni et al., 2013). MEPs facilitation by PAS was attenuated by oral administration of cortisol (Sale, Ridding and Nordstrom, 2008).

The aims of this study were, first of all, to explore the potential associations between salivary caffeine and corticosteroid concentrations with cortical excitability and plasticity aftereffects. Second, we examined the effects of caffeine, stimulation type, and time of day on the corticosteroid concentrations. We hypothesized that higher caffeine concentration is associated with higher cortical excitability. An increase of poststimulation caffeine and poststimulation corticosteroid concentrations inhibit plasticity aftereffects.

\subsection{MATERIAL AND METHODS}

\subsubsection{Participants}

Sixty participants were recruited from the students at the University of Göttingen. Experiment 1 comprised 30 caffeine-naïve subjects (15 male; age $23.6 \pm 3.3$ years (mean $\pm \mathrm{SD}$, range 19-31). Caffeine-naïve subjects were defined as those who never consumed coffee or abstained from coffee at least in the period of one year before studies. In addition they had been instructed to refrain from all caffeine-containing products at least five days before the experimental day. Thirty caffeine consumers took part in Experiment 2 (15 male; age $23.8 \pm 2.3$ years (mean $\pm \mathrm{SD}$, range 19-29). The participants in Experiment 2 had been instructed to refrain from all caffeine-containing products on the day of the experiment. All participants were screened for right-handedness (Edinburgh handedness inventory, Oldfield, 1971), caffeine consumption, a history of neurological or psychiatric disorders, or any contraindication to brain stimulation. An ECG was performed to rule out any risk for caffeine-induced cardiac arrhythmias. Medication history and consumption of alcohol and caffeine-containing products were recorded in every session. We included healthy male and female volunteers ( $\geq 18$ years) after obtaining a written consent. A history of neurological or psychiatric disease, left-handedness and regular consumption of alcohol and requirement for regular drug intake (except for hormonal contraceptives) were exclusion criteria in this study. The sessions with female participants took place in the period between menstruations (at least five days after the menses ended) in order to ensure constant oestrogen levels (Smith et al., 1999; Lee et al., 
2018). This study was approved by the local ethics committee and conducted in accordance with Declaration of Helsinki. It was registered in the ClinicalTrials.gov with the registration IDs NCT03720665 and NCT04011670.

\subsubsection{Transcranial Magnetic Stimulation (TMS)}

Motor evoked potentials (MEPs) were recorded from the right first dorsal interosseous muscle (FDI) with surface $\mathrm{Ag}-\mathrm{AgCl}$ electrodes in a belly-tendon montage. TMS was performed with a Magstim $200^{2}$ magnetic stimulator (Magstim Co. Ltd., Whitland, Wales, UK) and a D70 coil. The intersection of the line between nasion and inion, and the line between left and right periauricular was used as a landmark for reliably positioning the coil in the following sessions. The coil was held tangentially to the skull in a posterior-anterior (PA) direction at a $45^{\circ}$ angle pointing towards the right forehead. The electromyography signals were amplified, band-pass filtered ( $2 \mathrm{~Hz}-2 \mathrm{kHz})$, and digitized at a sampling rate of $5 \mathrm{kHz}$ with a micro $1401 \mathrm{AD}$ converter (Cambridge Electronic Design Ltd., Cambridge, UK). All data were stored on a hard disc for off-line analysis. A customized script was used to extract peak-to-peak amplitudes (Signal software version 4; Cambridge Electronic Design Ltd., Cambridge, UK). In each session, we determined the resting motor threshold (RMT), i.e. the lowest intensity required to elicit peak-to-peak MEPs of $\geq 50 \mu \mathrm{V}$ in five of ten stimuli. We recorded 25 stimuli to determine the intensity to produce approximately $1 \mathrm{mV}$ peak-to-peak MEPs $\left(\mathrm{MT}_{1 \mathrm{mV}}\right)$ and followed the protocols as described by Rossini and colleagues (Rossini et al., 2015).

\subsubsection{Paired Associative Stimulation (PAS)}

Two electrocardiography electrodes (ECG) were used to stimulate the right median nerve. The anodal electrode was attached over the median nerve at the wrist with the cathodal electrode $2 \mathrm{~cm}$ more proximally. The TMS coil was placed on the left M1, and a coil stand was used to maintain the coil position for the whole course of stimulation. In the PAS protocol, the median nerve was stimulated for 30 minutes at a frequency of $0.05 \mathrm{~Hz}$ with ninety non-painful electrical stimuli, constant current, $0.2 \mathrm{~ms}$ square pulses (pulse generator DS5, Digitimer, UK) (Stefan et al., 2000, 2002). Each electrical stimulus was followed by a single-pulse TMS with an inter-stimulus interval 
(ISI) of $25 \mathrm{~ms}$. We determined the individual threshold before the stimulation starts from a participant's subjective report. The threshold was determined as the least perceivable sensation of the electrical current on the stimulated median nerve. An actual stimulation intensity used for PAS was $300 \%$ of the perceptual threshold.

\subsubsection{Transcranial Alternating Current Stimulation (tACS)}

A battery-driven stimulator (NeuroConn GmbH, Illmenau, Germany) was used to deliver $140 \mathrm{~Hz}$ sinusoidal waveform through two conductive rubber electrodes. The active electrode $(4 \times 4 \mathrm{~cm})$ was placed over the left primary motor cortex at the position giving the best MEP response in the target FDI (M1), and the return electrode $(5 \times 7 \mathrm{~cm})$ was placed over the contralateral orbitofrontal cortex with the long edge above and parallel to the right eyebrow. The electrode was oriented so that the cable exiting from the short side led around to the right, while the cable of the M1 electrode was led to the back. We prepared the electrodes following the protocol of (Zulkifly, Merkohitaj and Paulus, 2020) in order to replicate their findings. Electrode cream was applied to the M1 electrode (Ten20, D.O. Weaver, Aurora, CO, USA), and a saline-soaked sponge was used for the return electrode. The stimulation duration was ten minutes with a current intensity of either $1 \mathrm{~mA}$ or $0.4 \mathrm{~mA}$. At the beginning of stimulation, the current was ramped up for five seconds and the stimulation maintained for ten minutes before it was ramped down for five seconds and immediately terminated. In the sham stimulation, the current was ramped up for five seconds and maintained the stimulation for 30 seconds before ramping down for five seconds. This 30 -second stimulation was intended to induce a similar skin sensation for the purpose of blinding. The impedance was kept below $10 \mathrm{k} \Omega$.

\subsubsection{Saliva sample collection and analysis}

Saliva samples were collected at the beginning (PRE) and at the end (POST) of each session using Salivette ${ }^{\circledR}$ Cortisol collection tubes (Sarstedt, Nümbrecht, Germany). The saliva was collected according to the manufacturer's instructions and was stored at $-20^{\circ} \mathrm{C}$ until assayed. The Salivette ${ }^{\circledR}$ tubes were centrifuged and the probes were used for caffeine, cortisone and cortisol analysis. For caffeine analysis, $50 \mu \mathrm{l}$ saliva was analysed, whereas $100 \mu \mathrm{l}$ saliva was analysed for cortisol and 
cortisone using high performance liquid chromatography (HPLC) and detection with tandem mass spectrometry. Reference substances (caffeine, cortisone, and cortisol) as well as deuterated internal standards (caffeine-D3 and cortisol-D4) were from Sigma (Deisenhofen, Germany). Cortisol was also used as internal standard for cortisone. After adding the internal standards, analytes were extracted with $1 \mathrm{ml}$ ethyl acetate: isopropanol (9:1) and the dried extract was redissolved in 0.1 formic acid prior to chromatography on a Brownlee SPP RP-amid, $2.7 \mu \mathrm{g}$, column (2.6 x $100 \mathrm{~mm})$. Detection of caffeine, caffeine-D3, cortisol, cortisol-D4 and cortisone was based on the parent/fragment $\mathrm{m} / \mathrm{z}$ ratios of 195.2/138, 198.1/138.0, 363.2/121.0, 367.2/121.0, and 361.2/163.0, respectively. The limit of quantification (LOQ) for caffeine was $10 \mathrm{ng} / \mathrm{ml}$, and $1 \mathrm{ng} / \mathrm{ml}$ for cortisol.

\subsubsection{Experimental design}

Both experiments in this study were randomized with a double-blind, cross-over design. The order of the interventions was assigned by a statistician who was not involved in the study.

Experiment 1 was designed to examine the relationship between salivary caffeine and corticosteroid concentrations with cortical plasticity induced by $140 \mathrm{~Hz}$ tACS and $25 \mathrm{~ms}$ PAS in caffeine-naïve subjects (Figure 4.1a). Before starting the experiment, the saliva sample was collected (prestimulation saliva), and the participant completed the questionnaire described above. They sat comfortably in a reclining chair, and the experiment started with the determination of the M1 stimulation spot (see above). The optimal position was marked and was used as a guide for reproducibly positioning the coil. The resting motor threshold (RMT) was determined with the coil over the identified optimal position following recent guidelines (Rossini et al., 2015). In the next step, we increased the stimulation intensity until a consistent MEP of approximately $1 \mathrm{mV}$ (peak to peak amplitude) $\left(\mathrm{MT}_{1 \mathrm{mV}}\right)$ was achieved. This intensity was used to record the baseline MEPs (PRE).

Intervention 1 started with the administration of a tablet containing either caffeine or placebo followed by a 45-minute waiting period for drug uptake in order to meet the peak saliva caffeine level which was reported to take longer with a capsule than coffee or cola (mean $\pm \mathrm{SD}=$ $67 \pm 7 \mathrm{~min}$ ) (Liguori, Hughes and Grass, 1997). During this period, the participants were allowed 
to read supplied materials, which were chosen to maintain wakefulness but not provoke arousal. Immediately after the 45-minute waiting period, Intervention 2 (tACS or PAS 25) was administered following the randomised stimulation protocols. The excitatory and inhibitory tACS protocols lasted for ten minutes, while the PAS 25 protocol was administered for 30 minutes for each protocol according to the randomization order. Aftereffects were measured by stimulating the designated cortical area. Poststimulation effects were recorded every five minutes at seven time points (Figure 4.1a). After the last measurement, the second saliva sample was collected (poststimulation saliva), and the participants filled out their questionnaires. These steps were followed in the same manner in all sessions.

Experiment 2 (Figure 4.1b) was designed to study the association between salivary corticosteroid concentrations with tACS aftereffects in caffeine consumers. All study protocols followed the same schedule as in Experiment 1 except that we changed the stimulation part in Intervention 2. It consisted of two stimulation and two time of day conditions in a randomized order: i) tACS (1.0 mA); morning, ii) tACS (1.0 mA); afternoon, iii) tACS (Sham); morning, iv) tACS (Sham); afternoon.

The morning session was conducted before 12:00 with the morning start time 09:41 \pm 0:49 (mean \pm S.D, range 07: $35-11: 33$ ), while the afternoon session was carried out after 12:00 and usually starting at 15:07 \pm 1:05 (mean \pm S.D, range 12:00 - 18:03). The sessions were performed blinded in a randomized order with at least five days between sessions to avoid carryover effects. 
a)

\begin{tabular}{|c|c|c|c|c|c|c|c|}
\hline 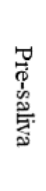 & $\begin{array}{l}\text { Determining M1 } \\
\text { stimulation spot }\end{array}$ & $\begin{array}{c}\text { Baseline } \\
\text { measurement } \\
(\mathrm{PRE})\end{array}$ & $\begin{array}{l}\text { Intervention } 1 \text { : medication } \\
\text { a) Caffeine tablet }(200 \mathrm{mg}) \\
\text { b) Placebo tablet }(200 \mathrm{mg})\end{array}$ & $\begin{array}{l}45 \mathrm{~min} \\
\text { break }\end{array}$ & $\begin{array}{l}\text { Intervention 2: stimulation } \\
\text { a) tACS } 140 \mathrm{~Hz}(1 \mathrm{~mA}) \\
\text { b) tACS } 140 \mathrm{~Hz}(0.4 \mathrm{~mA}) \\
\text { c) tACS } 140 \mathrm{~Hz} \text { (Sham) } \\
\text { d) PAS } 25\end{array}$ & $\begin{array}{l}\text { Post-effects } \\
\text { measurements } \\
\text { (PST0, PST5, } \\
\text { PST10, PST15, } \\
\text { PST20, PST25, } \\
\text { PST30) }\end{array}$ & 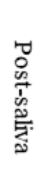 \\
\hline
\end{tabular}

b)

\begin{tabular}{|c|c|c|c|c|c|c|c|}
\hline 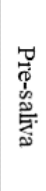 & $\begin{array}{l}\text { Determining M1 } \\
\text { stimulation spot }\end{array}$ & $\begin{array}{c}\text { Baseline } \\
\text { measurement } \\
(\mathrm{PRE})\end{array}$ & $\begin{array}{l}\text { Intervention } 1 \text { : medication } \\
\text { a) Caffeine tablet }(200 \mathrm{mg}) \\
\text { b) Placebo tablet }(200 \mathrm{mg})\end{array}$ & $\begin{array}{l}45 \mathrm{~min} \\
\text { break }\end{array}$ & $\begin{array}{l}\text { Intervention 2: stimulation } \\
\text { a) tACS } 140 \mathrm{~Hz}(1 \mathrm{~mA}) \text {; morning } \\
\text { b) tACS } 140 \mathrm{~Hz}(1 \mathrm{~mA}) \text {; evening } \\
\text { c) tACS } 140 \mathrm{~Hz} \text { (Sham); morning } \\
\text { d) tACS } 140 \mathrm{~Hz} \text { (Sham); evening }\end{array}$ & $\begin{array}{l}\text { Post-effects } \\
\text { measurements } \\
\text { (PST0, PST5, } \\
\text { PST10, PST15, } \\
\text { PST20, PST25, } \\
\text { PST30) }\end{array}$ & 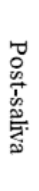 \\
\hline
\end{tabular}

Figure 4.1 Experimental design.

The experiment started and ended with the saliva sample collection. The study design follows the same steps in a sequence, which includes the optimal stimulation position over the left motor cortex (M1) for the right FDI muscle, baseline measurements of the induced MEPs, and administration of the tablet with caffeine or placebo in a random order. Experiment 1, i) explored the relationship between salivary caffeine and corticosteroid concentrations with cortical plasticity induced by 140 $\mathrm{Hz}$ tACS, and PAS 25 in the caffeine-naïve group, ii) examined the effects of caffeine and stimulation on corticosteroid concentrations: stimulation began after a 45-minute waiting period after the tablet was ingested.

In Experiment 1, tACS was applied for ten minutes and PAS 25 for 30 minutes to the defined M1 spot. Beginning immediately after the stimulation, the poststimulation effects were measured at fiveminute intervals for 30 minutes.

Experiment 2, i) explored the association between caffeine concentrations, salivary corticosteroid concentrations and the tACS aftereffects in the caffeine consumer group, ii) investigated the effects of caffeine, stimulation and time of day on the salivary concentrations of corticosteroids: The preparations were the same as for Experiment 1, but only $140 \mathrm{~Hz}$ tACS was performed. The morning session was conducted before 12:00, and the afternoon session was conducted after 12:00. The poststimulation measurements were as in Experiment 1.

The order of the sessions in both Experiment 1 and 2 were randomized, and each session was conducted with an at least five-day interval between sessions. Abbreviations: $\mathrm{MEP}=$ motor evoked potential; tACS = transcranial alternating current stimulation; PAS = paired associative stimulation; $\mathrm{M} 1$ = primary motor cortex PRE = baseline; $\mathrm{PST}=$ poststimulation . 


\subsubsection{Data analysis and statistics}

The peak-to-peak MEP amplitudes were evaluated using a customized Signal software script (Signal version 4.08, Cambridge Electronic Design Ltd., Cambridge UK). An average of MEPs were obtained from $25 \mathrm{MEP}$ trials at each measurement time. Baseline MEPs were the average of the starting measurement (PRE), POST 1 was the average of the measurements at $0 \mathrm{~min}, 5 \mathrm{~min}$, $10 \mathrm{~min}, 15 \mathrm{~min}$ poststimulation, POST 2 was the average of the measurements at $20 \mathrm{~min}, 25 \mathrm{~min}$ and 30 min poststimulation and PST30 was the average of the poststimulation measurements at 30 $\min$. The salivary caffeine concentrations were given as milligram per litre (mg/L), and the salivary cortisone and cortisol concentrations were reported as microgram per liter $(\mu \mathrm{g} / \mathrm{L})$. A total sum of salivary cortisone and cortisol concentrations were obtained to reflect the total corticosteroid concentrations $(\mu \mathrm{g} / \mathrm{L})$. SPSS was used for statistical analysis (IBM SPSS statistics 26; IBM Corp., Armonk, NY, USA). Data distribution was tested, and non-parametric tests were used if the normality assumption was violated. Prestimulation and poststimulation caffeine and corticosteroid concentrations were $\log _{10}$-transformed for further analysis.

In both experiments, we performed a correlation analysis using Spearman correlation or Pearson correlation to evaluate the relationship between normalized prestimulation caffeine, prestimulation corticosteroid concentrations and motor thresholds and also baseline MEP amplitudes. We correlated normalized poststimulation caffeine and poststimulation corticosteroid concentrations in each condition with motor cortex excitability using POST 1, POST 2 and PST30 data, which indicates an exact timing of salivary samples collection. In Experiment 1, a repeated measures (rm) ANOVA was used to examine the effects of normalized salivary caffeine and corticosteroid concentrations in all conditions with gender as a between-subject factor, and time, drug and stimulation as within-subject factors (TIME 2 levels $\mathrm{X} \mathrm{DRUG}_{2 \text { levels}}$ ) for the PAS 25 dataset and (TIME 2 levels $x$ DRUG $_{2 \text { levels }}$ x STIMULATION 3 levels) for the tACS dataset. A post hoc t-test was used to compare the significant effects of rmANOVAs. We defined the sample collection times as "morning" when the sample was collected between 08:00 and 11:59, and as "afternoon" when collected between 12:00 and 20:00. RmANOVAs were also used to determine the effects of within- and between-subject factors on corticosteroids in Experiment 2. We included gender as a between-subject factor in our rmANOVA models. Next, measurement time, stimulation, time of 
day and drug were considered as within-subject factors $\left(\mathrm{TIME}_{2}\right.$ levels $\mathrm{X}$ STIMULATION 2 levels $\mathrm{X}$ $\mathrm{DAY}_{2 \text { levels }} \mathrm{x} \mathrm{DRUG}_{2}$ level). We excluded caffeine raw data sets that had prestimulation concentrations with a similar or even higher than average concentrations after $200 \mathrm{mg}$ caffeine consumption. (i.e., caffeine-naïve: 3 raw data sets were excluded, prestimulation caffeine concentration of $\geq 2.0 \mathrm{mg} / \mathrm{L}$; caffeine-adapted consumers: 6 data sets were excluded, prestimulation caffeine concentration of $\geq 3.0 \mathrm{mg} / \mathrm{L}$ ).

A cortisol data point with a concentration below the limit of quantification (LOQ) was assigned a value of $0.5 \mu \mathrm{g} / \mathrm{L}$ equal to half the LOQ value. Undetectable cortisol concentrations, i.e. below the level of detection (LOD) were recorded as missing values. For the ANOVA analyses sphericity was assessed with Mauchly's test of sphericity, and Greenhouse-Geisser correction was used if sphericity was violated. The Bonferroni correction was used for multiple comparisons, and subsequent post hoc comparisons were performed with paired t-tests or Wilcoxon test. All data were presented as mean \pm standard error of means (SEM), unless otherwise specified. The significance level was set at $\mathrm{p} \leq 0.05$.

\subsection{RESULTS}

\subsubsection{Salivary caffeine concentrations}

Elevated salivary caffeine concentrations confirmed that the participants had received the correct tablets both in Experiment 1 and Experiment 2. In the caffeine-naïve group, the caffeine concentration in saliva had increased at 2 to 2.5 hours after caffeine ingestion from a baseline median of $0.05-0.13 \mathrm{mg} / \mathrm{L}$ to a median POST concentration of $3.23-3.48 \mathrm{mg} / \mathrm{L}$ in all conditions with caffeine (See Table 4.1). Caffeine concentrations above zero in the caffeine naïve group may originate from various nutritional sources since we did not apply a completely controlled/standardized diet at the day before the study and at the day of study. Highest caffeine

concentration in that group was $1.39 \mathrm{mg} / \mathrm{L}$, corresponding to a dose of about $69.5 \mathrm{mg}$ (calculated by multiplying with a volume of 50 Liter) which is compatible with caffeine contents, for instance, in chocolate containing foods or green or black tea. 
Baseline salivary caffeine levels were higher in the caffeine consumer group with a median of $0.27-0.51 \mathrm{mg} / \mathrm{L}$ compatible with a caffeine half-life of about 5 hours and the fact, that this group was only not allowed to drink coffee at the day of study but may have drunk coffee at the day before. They increased after caffeine administration with a median POST concentration of $3.56-3.80 \mathrm{mg} / \mathrm{L}$ in conditions with caffeine. (See Table 4.1).

Table 4.1 Caffeine concentrations in saliva

\begin{tabular}{|c|c|c|c|}
\hline & Condition & $\begin{array}{c}\text { Caffeine concentration } \\
\text { PRE }(\mathrm{mg} / \mathrm{L})\end{array}$ & $\begin{array}{c}\text { Caffeine concentration } \\
\text { POST }(\mathrm{mg} / \mathrm{L})\end{array}$ \\
\hline \multirow{10}{*}{$\begin{array}{l}\text { Experiment } 1 \\
\text { (caffeine naïve) }\end{array}$} & a) tACS & & \\
\hline & $1.0 \mathrm{~mA}$, placebo & $0.10(0.02-0.33)$ & $0.07(0.02-0.23)$ \\
\hline & $1.0 \mathrm{~mA}$, caffeine & $0.07(0.02-0.21)$ & $3.48(2.84-4.18)$ \\
\hline & $0.4 \mathrm{~mA}$, placebo & $0.07(0.03-0.24)$ & $0.06(0.01-0.20)$ \\
\hline & $0.4 \mathrm{~mA}$, caffeine & $0.05(0.02-0.24)$ & $3.23(2.73-4.38)$ \\
\hline & Sham, placebo & $0.07(0.03-0.19)$ & $0.05(0.02-0.16)$ \\
\hline & Sham, caffeine & $0.07(0.04-0.20)$ & $3.73(2.88-4.50)$ \\
\hline & b) PAS 25 & & \\
\hline & Placebo & $0.13(0.01-0.44)$ & $0.08(0.01-0.37)$ \\
\hline & Caffeine & $0.05(0.01-0.23)$ & $3.40(2.77-4.21)$ \\
\hline \multirow{10}{*}{$\begin{array}{l}\text { Experiment } 2 \\
\text { (caffeine consumers) }\end{array}$} & a) tACS (1.0 mA) & & \\
\hline & morning, placebo & $0.37(0.15-0.72)$ & $0.29(0.10-0.47)$ \\
\hline & morning, caffeine & $0.41(0.22-0.71)$ & $3.76(2.88-4.41)$ \\
\hline & afternoon, placebo & $0.18(0.09-0.61)$ & $0.16(0.06-0.53)$ \\
\hline & afternoon, caffeine & $0.35(0.13-0.67)$ & $3.77(2.68-4.47)$ \\
\hline & b) tACS (Sham) & & \\
\hline & morning, placebo & $0.32(0.17-0.70)$ & $0.25(0.11-0.60)$ \\
\hline & morning, caffeine & $0.27(0.12-0.85)$ & $3.80(2.74-4.51)$ \\
\hline & afternoon, placebo & $0.37(0.16-0.75)$ & $0.24(0.10-0.52)$ \\
\hline & afternoon, caffeine & $0.51(0.16-0.97)$ & $3.56(2.86-4.35)$ \\
\hline
\end{tabular}

Values are given as median and interquantile range. PRE $=$ before experiment; POST $=$ after experiment

\subsubsection{Salivary cortisone, cortisol and total corticosteroid concentrations}

In Experiment 1 with caffeine-naïve participants, the median salivary cortisone concentration decreased significantly from a baseline concentration of $6.81-8.59 \mu \mathrm{g} / \mathrm{L}$ to a median POST concentration of $3.73-6.89 \mu \mathrm{g} / \mathrm{L}$ in all conditions (Supplementary Table 4.1). Similarly, in Experiment 2 with caffeine consumers, the median baseline salivary cortisone concentration was $5.62-10.08 \mu \mathrm{g} / \mathrm{L}$ and decreased significantly to a median POST concentration of $3.23-5.65 \mu \mathrm{g} / \mathrm{L}$ (Supplementary Table 4.1). 
In the caffeine-naïve group, baseline salivary cortisol concentrations were significantly higher with a median concentration of $1.28-2.08 \mu \mathrm{g} / \mathrm{L}$ compared to the POST median concentration of $0.50 \mu \mathrm{g} / \mathrm{L}$. (Supplementary Table 4.2). In caffeine consumers, baseline salivary cortisol concentrations were $0.50-2.27 \mu \mathrm{g} / \mathrm{L}$ and were significantly lower at the POST measurement with a median concentration of 0.50 (Supplementary Table 4.2).

A sum of corticosteroids concentration (i.e. a total of cortisone and cortisol) were higher at the baseline compared to poststimulation corticosteroids in both groups. In Experiment 1 with caffeine-naïve group, the median prestimulation corticosteroids was $8.41-10.60 \mu \mathrm{g} / \mathrm{L}$ and decreased to a median poststimulation concentration of $4.25-8.85 \mu \mathrm{g} / \mathrm{L}$ (Table 2). In caffeineadapted consumers, baseline prestimulation corticosteroids concentration was $6.60-12.35 \mu \mathrm{g} / \mathrm{L}$ and reduced at the poststimulation measurement with a median concentration of $4.60-6.27 \mu \mathrm{g} / \mathrm{L}$ (Table 4.2).

Table 4.2 Corticosteroids concentration in saliva

\begin{tabular}{llll}
\hline & Condition & $\begin{array}{l}\text { Total corticosteroids } \\
\text { concentration PRE }(\boldsymbol{\mu g} / \mathbf{L})\end{array}$ & $\begin{array}{l}\text { Total corticosteroids } \\
\text { concentration Post }(\boldsymbol{\mu g} / \mathbf{L})\end{array}$ \\
\hline $\begin{array}{l}\text { Experiment } 1 \\
\text { (caffeine-naïve) }\end{array}$ & tACS & $10.19(5.16-12.95)$ & $5.57(3.81-7.19)$ \\
& 1) $1.0 \mathrm{~mA}$, placebo & $9.20(5.88-16.66)$ & $5.82(3.97-11.01)$ \\
& 2) $1.0 \mathrm{~mA}$, caffeine & $8.96(5.44-15.83)$ & $5.61(4.15-6.71)$ \\
3) $0.4 \mathrm{~mA}$, placebo & $8.53(5.40-14.84)$ & $5.93(4.52-10.88)$ \\
& 4) $0.4 \mathrm{~mA}$, caffeine & $9.56(7.26-15.52)$ & $4.25(3.78-7.82)$ \\
& 5) Sham, placebo & $10.60(6.36-15.82)$ & $7.39(4.98-9.92)$ \\
6) Sham, caffeine & & \\
PAS 25 & $10.58(5.26-15.33)$ & $4.98(3.60-7.24)$ \\
Experiment 2 & 7) Placebo & & $8.85(6.35-5.51)$ \\
8) Caffeine & & \\
& tACS (1.0 mA) & $10.94(5.99-17.82)$ & $5.92(4.67-8.39)$ \\
& 1) morning, placebo & $12.22(7.26-15.64)$ & $6.27(4.70-8.42)$ \\
2) morning, caffeine & $6.77(5.57-10.75)$ & $4.60(3.56-6.02)$ \\
3) afternoon, placebo & $6.60(5.55-15.11)$ & $5.74(4.10-6.78)$ \\
4) afternoon, caffeine & & \\
tACS (Sham) & $11.18(8.23-14.69)$ & $5.66(4.32-7.31)$ \\
5) morning, placebo & $12.35(9.39-15.66)$ & $6.15(4.97-9.82)$ \\
6) morning, caffeine & $7.39(5.10-11.99)$ & $4.81(3.91-7.24)$ \\
7) afternoon, placebo & $7.29(4.76-9.99)$ & $4.94(3.89-6.04)$ \\
8) afternoon, caffeine & &
\end{tabular}




\subsubsection{Relationship between caffeine and corticosteroids concentration with motor cortex excitability}

We grouped the data in all conditions for both prestimulation caffeine and prestimulation corticosteroids. The relationship between PRE caffeine and PRE corticosteroids concentrations with motor thresholds (i.e., resting motor threshold (RMT), $1 \mathrm{mV}$ MEP motor threshold $\left(\mathrm{MT}_{1 \mathrm{mV}}\right)$ and baseline MEP amplitudes (MEPPRE) were examined. In the caffeine naïve group, there was no correlation between the prestimulation caffeine concentration with motor thresholds and baseline MEPs. On the other hand, there was a positive correlation between prestimulation corticosteroid levels with the resting motor threshold (Pearson correlation, $r=0.19, p<0.05$; Table 4.3) whereas higher prestimulation caffeine concentrations in the afternoon were associated with lower motor thresholds and higher baseline MEPs (i.e., a) RMT: Pearson correlation, $r=-0.34, p<0.05$; b)

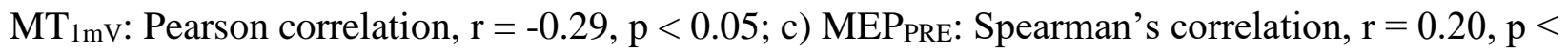
0.05). In other words elevation of the prestimulation caffeine was associated with increase in baseline cortical excitability in the afternoon (Table 4.3) whereas in the morning sessions no correlation between prestimulation caffeine concentrations with motor thresholds and baseline MEPs could be seen (Table 4.3).

Table 4.3 The correlations of log transformed prestimulation caffeine and prestimulation corticosteroids concentrations with motor thresholds and baseline MEP amplitudes (MEP PRE)

\begin{tabular}{|c|c|c|c|c|c|c|}
\hline \multirow[t]{2}{*}{ Condition } & \multicolumn{2}{|c|}{ RMT (\% MSO) } & \multicolumn{2}{|c|}{ MT $_{1 m V}(\%$ MSO) } & \multicolumn{2}{|c|}{ MEPPRE (mV) } \\
\hline & $\mathrm{r}$ & $\mathrm{p}$ & $\mathrm{r}$ & $\mathrm{p}$ & $\mathrm{r}$ & $\mathrm{p}$ \\
\hline \multicolumn{7}{|l|}{$\begin{array}{l}\text { 1) Experiment } 1 \text { (caffeine- } \\
\text { naïve) }\end{array}$} \\
\hline $\begin{array}{l}\text { prestimulation } \\
\text { caffeine }(\mathrm{mg} / \mathrm{L})\end{array}$ & -0.11 & 0.12 & -0.10 & 0.15 & -0.10 & 0.17 \\
\hline $\begin{array}{l}\text { prestimulation } \\
\text { corticosteroids } \\
(\mu \mathrm{g} / \mathrm{L})\end{array}$ & 0.19 & $0.004 *$ & 0.00 & 0.96 & 0.07 & 0.31 \\
\hline \multicolumn{7}{|l|}{$\begin{array}{l}\text { 2) Experiment } 2 \text { (caffeine- } \\
\text { adapted consumers) }\end{array}$} \\
\hline \multicolumn{7}{|l|}{ a. Morning sessions } \\
\hline $\begin{array}{l}\text { prestimulation } \\
\text { caffeine }(\mathrm{mg} / \mathrm{L})\end{array}$ & -0.03 & 0.76 & 0.02 & 0.80 & -0.01 & 0.95 \\
\hline $\begin{array}{l}\text { prestimulation } \\
\text { corticosteroids } \\
(\mu \mathrm{g} / \mathrm{L})\end{array}$ & 0.11 & 0.25 & 0.01 & 0.92 & -0.05 & 0.55 \\
\hline b. Afternoon sessions & & & & & & \\
\hline prestimulation & -0.34 & $<0.001 *$ & -0.29 & $0.002 *$ & 0.20 & $0.04 * \wedge$ \\
\hline
\end{tabular}


caffeine (mg/L)

prestimulation

$-0.17$

0.08

$-0.20$

$0.04 *$

0.14

0.14

corticosteroids

$(\mu \mathrm{g} / \mathrm{L})$

Abbreviations: $\mathrm{RMT}=$ resting motor threshold; $\% \mathrm{MSO}=\%$ of maximum stimulator output; $\mathrm{MT}_{1 \mathrm{mV}}=$ motor threshold which produces $1 \mathrm{mV}$ MEP; MEP $_{\mathrm{PRE}}=$ baseline motor evoked potential; tACS $=$ transcranial alternating current stimulation; PAS $=$ paired associative stimulation. $* \mathrm{p}<0.05$; $^{\wedge}$ Spearman’s correlation

We also examined the association between poststimulation caffeine, poststimulation corticosteroids concentrations and MEPs at the first 15 min poststimulation measurement (POST 1), MEPs at the last 15 min poststimulation measurement (POST 2) and MEPs at the 30 minutes poststimulation measurement point (PST30). Any relationships at POST 1 and POST 2 may explain the time dynamic changes of those salivary concentrations with cortical excitability. Next, the associations at PST30 provided more useful information on the hormonal activity at the time of sample collection with cortical excitability. In caffeine-naïve participants, an increased in poststimulation caffeine concentrations after sham was associated with deceased in poststimulation MEPs at POST1 (Pearson correlation, $\mathrm{r}=-0.42, \mathrm{p}<0.05$; Table 4.4). In other stimulation conditions, there were no significant correlations between poststimulation caffeine concentrations and poststimulation MEPs. This indicates that caffeine itself had an effect on the MEPs. Table 4.5 shows there were no correlations between poststimulation corticosteroid concentrations and poststimulation MEPs after caffeine in all conditions.

Table 4.4 Experiment 1 (caffeine-naïve): The correlations of log transformed poststimulation caffeine concentrations with MEP amplitudes at the poststimulation measurement 1 (POST 1), poststimulation measurement 2 (POST 2) and at 30 minutes poststimulation measurement (PST30)

\begin{tabular}{lcccccc}
\hline Condition & \multicolumn{2}{c}{ POST 1 } & \multicolumn{2}{c}{ POST 2 } & \multicolumn{2}{c}{ PST30 } \\
& $\mathrm{r}$ & $\mathrm{p}$ & $\mathrm{r}$ & $\mathrm{p}$ & $\mathrm{r}$ & $\mathrm{p}$ \\
tACS & & & & & & \\
1) $1.0 \mathrm{~mA}$, placebo & -0.18 & 0.39 & -0.14 & 0.51 & -0.19 & 0.35 \\
2) $1.0 \mathrm{~mA}$, caffeine & -0.16 & 0.39 & -0.28 & 0.13 & -0.29 & 0.13 \\
3) $0.4 \mathrm{~mA}$, placebo & 0.19 & 0.38 & 0.03 & 0.90 & 0.08 & 0.72 \\
4) $0.4 \mathrm{~mA}$, caffeine & 0.05 & 0.80 & 0.06 & 0.74 & 0.03 & 0.88 \\
5) Sham, placebo & -0.17 & 0.45 & -0.25 & 0.25 & -0.16 & 0.48 \\
6) Sham, caffeine & -0.42 & $0.02^{*}$ & -0.25 & 0.20 & -0.24 & 0.21 \\
PAS 25 & & & & & & \\
7) Placebo & -0.25 & 0.24 & -0.42 & $0.04^{*}$ & -0.33 & 0.11 \\
8) Caffeine & 0.04 & 0.84 & 0.08 & 0.68 & -0.02 & 0.93 \\
\hline
\end{tabular}

Abbreviations: POST $1=$ average of first 15 min post stimulation measurements; POST $2=$ average of last 15 min post stimulation measurements; PST30 = MEP amplitudes at the 30 minutes poststimulation measurement; $\mathrm{tACS}=$ transcranial alternating current stimulation; PAS $=$ paired associative stimulation. $* \mathrm{p}<0.05$ 
Table 4.5 Experiment 1 (caffeine-naïve): The correlations of log transformed poststimulation corticosteroid concentrations with MEP amplitudes at the poststimulation measurement 1 (POST 1), poststimulation measurement 2 (POST 2) and at 30 minutes poststimulation measurement (PST30)

\begin{tabular}{lcccccc}
\hline Condition & \multicolumn{2}{c}{ POST 1 } & \multicolumn{2}{c}{ POST 2 } & \multicolumn{2}{c}{ PST30 } \\
& $\mathrm{r}$ & $\mathrm{p}$ & $\mathrm{r}$ & $\mathrm{p}$ & $\mathrm{r}$ & $\mathrm{p}$ \\
tACS & & & & & & \\
1) $1.0 \mathrm{~mA}$, placebo & 0.27 & 0.19 & 0.28 & 0.18 & 0.24 & 0.26 \\
2) $1.0 \mathrm{~mA}$, caffeine & 0.33 & 0.09 & 0.28 & 0.15 & 0.28 & 0.16 \\
3) $0.4 \mathrm{~mA}$, placebo & -0.17 & 0.39 & -0.01 & 0.97 & -0.11 & 0.60 \\
4) $0.4 \mathrm{~mA}$, caffeine & 0.29 & 0.16 & 0.26 & 0.20 & 0.18 & 0.39 \\
5) Sham, placebo & 0.43 & $0.03^{* \wedge}$ & 0.02 & 0.94 & 0.04 & 0.84 \\
6) Sham, caffeine & 0.08 & 0.71 & 0.16 & 0.43 & 0.06 & 0.77 \\
PAS 25 & & & & & & \\
7) Placebo & 0.14 & 0.48 & 0.15 & 0.44 & 0.28 & 0.17 \\
8) Caffeine & 0.18 & 0.35 & 0.17 & 0.39 & -0.21 & 0.29 \\
\hline
\end{tabular}

Abbreviations: POST $1=$ average of first 15 min post stimulation measurements; POST $2=$ average of last $15 \mathrm{~min}$ post stimulation measurements; PST30 = MEP amplitudes at the 30 minutes poststimulation measurement; $\mathrm{tACS}=$ transcranial alternating current stimulation; PAS $=$ paired associative stimulation. $* \mathrm{p}<0.05$

In caffeine-adapted consumers, after caffeine intake, we did not find any relationships of poststimulation caffeine concentrations with plasticity aftereffects (Table 4.6). Similarly, there were no associations between poststimulation corticosteroids and poststimulation MEPs in all conditions (Table 4.7).

Table 4.6 Experiment 2 (caffeine consumers): The correlations of log transformed poststimulation caffeine concentrations with MEP amplitudes at the poststimulation measurement 1 (POST 1), poststimulation measurement 2 (POST 2) and at 30 minutes poststimulation measurement (PST30)

\begin{tabular}{lcccccc}
\hline Condition & \multicolumn{2}{c}{ POST 1 } & \multicolumn{2}{c}{ POST 2 } & \multicolumn{2}{c}{ PST30 } \\
\hline & $\mathrm{r}$ & $\mathrm{p}$ & $\mathrm{r}$ & $\mathrm{p}$ & $\mathrm{r}$ & $\mathrm{p}$ \\
1) tACS (1.0 mA); afternoon & & & & & & \\
placebo & 0.29 & 0.13 & 0.39 & $0.03^{*}$ & 0.29 & 0.12 \\
caffeine & 0.28 & 0.13 & 0.19 & 0.32 & 0.17 & 0.38 \\
$\begin{array}{l}\text { 2) tACS (1.0 mA); morning } \\
\text { placebo }\end{array}$ & -0.36 & 0.054 & -0.29 & 0.12 & -0.30 & 0.11 \\
caffeine & 0.10 & 0.60 & 0.07 & 0.70 & -0.25 & 0.19 \\
3) tACS (Sham); afternoon & & & & & & \\
placebo & 0.33 & 0.08 & 0.10 & 0.59 & -0.05 & 0.78 \\
caffeine & -0.13 & 0.50 & 0.04 & 0.85 & -0.20 & 0.29 \\
4) tACS (Sham); morning & & & & & & \\
placebo & 0.13 & 0.50 & 0.10 & 0.58 & 0.14 & 0.47 \\
caffeine & 0.06 & 0.77 & -0.02 & 0.93 & -0.04 & 0.86 \\
\hline
\end{tabular}


Abbreviations: POST 1 = average of first 15 min post stimulation measurements; POST 2 = average of last 15 min post stimulation measurements; PST30 = MEP amplitudes at the 30 minutes poststimulation measurement; tACS = transcranial alternating current stimulation; PAS $=$ paired associative stimulation. $* \mathrm{p}<0.05$

Table 4.7 Experiment 2 (caffeine consumers): The correlations of $\log$ transformed poststimulation corticosteroid concentrations with MEP amplitudes at the poststimulation measurement 1 (POST 1), poststimulation measurement 2 (POST 2) and at 30 minutes poststimulation measurement (PST30)

\begin{tabular}{lcccccc}
\hline Condition & \multicolumn{2}{c}{ POST 1 } & \multicolumn{2}{c}{ POST 2 } & \multicolumn{2}{c}{ PST30 } \\
\hline & $\mathrm{r}$ & $\mathrm{p}$ & $\mathrm{r}$ & $\mathrm{p}$ & $\mathrm{r}$ & $\mathrm{p}$ \\
1) tACS (1.0 mA); afternoon & & & & & & \\
placebo & 0.20 & 0.37 & 0.10 & 0.67 & 0.15 & 0.49 \\
caffeine & 0.01 & 0.98 & 0.06 & 0.80 & -0.02 & 0.92 \\
2) tACS (1.0 mA); morning & & & & & & \\
placebo & -0.06 & 0.78 & -0.31 & 0.13 & -0.33 & 0.10 \\
caffeine & 0.08 & 0.69 & -0.18 & 0.37 & -0.03 & 0.87 \\
3) tACS (Sham); afternoon & & & & & & \\
placebo & 0.07 & 0.77 & 0.03 & 0.91 & 0.02 & 0.91 \\
caffeine & 0.38 & 0.054 & 0.31 & 0.13 & 0.31 & 0.13 \\
4) tACS (Sham); morning & & & & & & \\
placebo & -0.03 & 0.88 & 0.04 & 0.84 & -0.08 & 0.70 \\
caffeine & -0.01 & 0.95 & -0.01 & 0.95 & 0.31 & 0.13 \\
\hline
\end{tabular}

Abbreviations: POST $1=$ average of first $15 \mathrm{~min}$ post stimulation measurements; POST $2=$ average of last $15 \mathrm{~min}$ post stimulation measurements; PST30 = MEP amplitudes at the 30 minutes poststimulation measurement; $\mathrm{tACS}=$ transcranial alternating current stimulation; PAS $=$ paired associative stimulation. $* p<0.05$

\subsubsection{Effects of caffeine, stimulation and time of day on circulating corticosteroids}

In caffeine-naïve subjects rmANOVA revealed a significant interaction between the effects of time of measurement and caffeine consumption on the salivary corticosteroids concentration both in PAS 25 and tACS condition $(\mathrm{p}<0.05)$. The main effect of time of measurement was significant ( $\mathrm{p}<0.001)$. In tACS conditions, we found a significant interaction between the effects of time, stimulation, drug and gender. There was no main effect of stimulation and no interaction between the effects of stimulation and caffeine on corticosteroids concentration (Supplementary Table 3). The post hoc t-test showed that salivary poststimulation corticosteroid concentrations were significantly higher with caffeine than without in the $0.4 \mathrm{~mA}$ condition $(\mathrm{t}(23)=-2.12, \mathrm{p}=0.045)$ and in the PAS 25 condition $(\mathrm{t}(25)=-2.27, \mathrm{p}=0.03)$. (Figure $2 \mathrm{a})$.

In the caffeine consumers, there was a main effect of time of measurement on the salivary corticosteroid concentrations $(\mathrm{p}<0.001)$ without main effects of stimulation, time of day and caffeine. We found the interaction effects between time of measurement and gender $(\mathrm{p}<0.05)$ and 
an interaction between the effects of stimulation, time of day and caffeine consumption $(\mathrm{p}<0.05)$. Also, an interaction between the effects of time of measurement, time of day and gender was also significant $(\mathrm{p}<0.05)$. There were no significant interactions between the effects of stimulation and time of day, no interactions between stimulation and caffeine and no interaction between the effects of time of day and caffeine. (Supplementary Table 4). In the afternoon sessions, the average prestimulation corticosteroids was higher in men than in women (Men: $11.01 \pm 6.37$; Women: 6.87 $\pm 3.00 ; \mathrm{t}(99.93)=4.27, \mathrm{p}<0.001)$. A similar difference was observed in the average of poststimulation corticosteroids (Men: $6.82 \pm 3.30$; Women: $4.52 \pm 2.17$; $(93)=4.84$, p < 0.001). Figure $2 b$ shows that the poststimulation corticosteroids concentration after Sham in the caffeine group were significantly higher in the morning than in the afternoon $(\mathrm{t}(23)=2.09, \mathrm{p}=0.048)$. Caffeine changes the circulating corticosteroid concentration in the morning as shown by higher poststimulation corticosteroids concentration after caffeine than after placebo $(\mathrm{t}(25)=3.15, \mathrm{p}=$ 0.004). 
a)

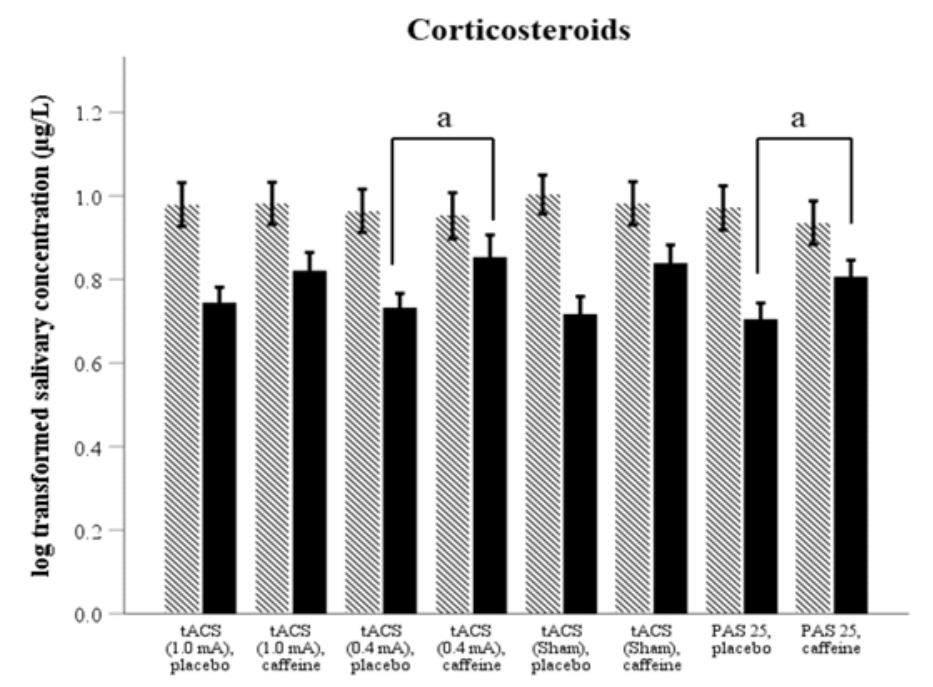

Condition b)

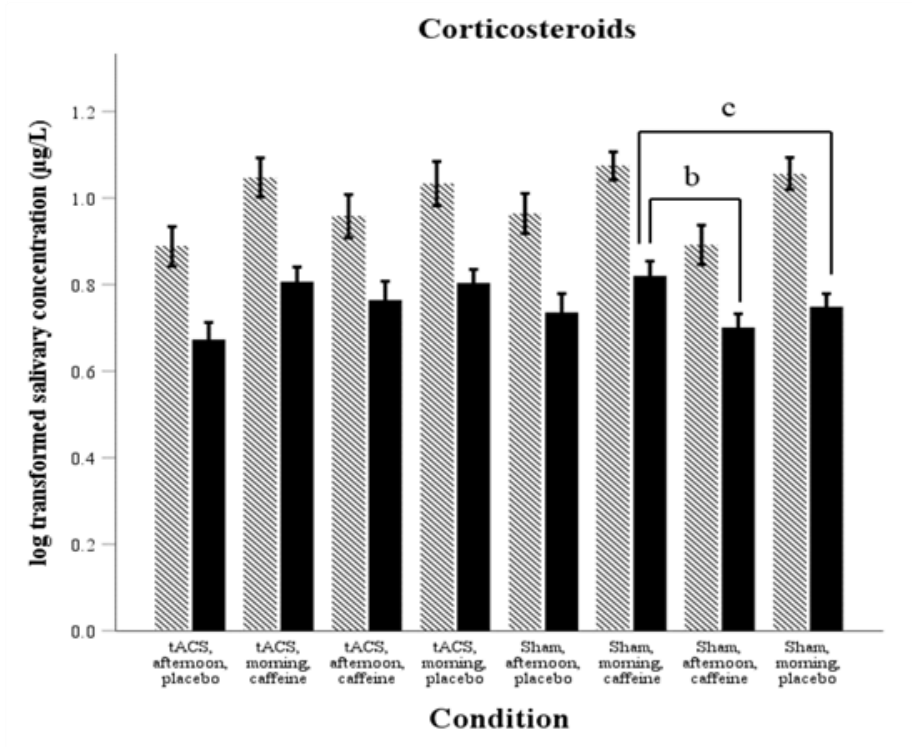

Figure 4.2 Salivary concentrations of corticosteroids in all conditions.

The figures in the left panel represent the data from the caffeine-naïve and those in the right panel represent the data from the caffeine consumer group.

a) Poststimulation salivary corticosteroid concentrations after $0.4 \mathrm{~mA}$ tACS and after PAS 25 were significantly higher with caffeine than with placebo. b) Poststimulation salivary corticosteroid concentrations in sham conditions were significantly higher after caffeine consumption in the morning session than in the afternoon. Caffeine increased poststimulation corticosteroids as shown by higher concentrations after caffeine than after placebo in the sham morning. $* \mathrm{p}<0.05 ; \mathrm{a}=$ poststimulation saliva measurement with placebo compared to caffeine; $\mathrm{b}=$ poststimulation saliva measurement with caffeine after sham in the morning compared to in the afternoon; $\mathrm{c}=$ poststimulation corticosteroid concentrations in the sham morning after caffeine compared to placebo. Abbreviations: tACS $=$ transcranial alternating current stimulation; PAS = paired associative stimulation; $\mathrm{PRE}=$ prestimulation saliva measurement; $\mathrm{POST}=$ poststimulation saliva measurement. 


\subsection{DISCUSSION}

The main purpose of this study was to investigate the relationships between salivary caffeine, salivary corticosteroids concentrations and cortical excitability and plasticity aftereffects on the motor cortex of caffeine-naïve and caffeine consuming participants. Several findings emerged: In the afternoon sessions in caffeine consumers, higher prestimulation caffeine concentrations were associated with higher cortical excitability as shown by lower in the motor thresholds and higher baseline MEP amplitudes. Also here in parallel, higher prestimulation corticosteroid concentration was correlated with a decrease in motor threshold but not significantly increase in MEP baseline amplitudes. A reverse relationship was observed in caffeine-naïve subjects. Here a higher prestimulation corticosteroid concentration was associated with an increase in motor threshold and higher poststimulation caffeine concentrations were associated with a decrease in poststimulation MEPs. No relationships between poststimulation caffeine concentration and poststimulation corticosteroid concentrations were found with plasticity aftereffects both in caffeine-naïve and caffeine-adapted subjects. Caffeine exerted its effect by increasing the corticosteroid concentrations, but neither changes in poststimulation caffeine nor poststimulation corticosteroid concentrations did correlate with plasticity aftereffects. Since the motor cortex is more excitable in the afternoon sessions, our result suggest that morning experimental sessions need more stimulation intensity for inducing plasticity aftereffects because of low baseline excitability thresholds.

Salivary caffeine concentrations in our study were slightly lower than in two studies by Lovallo and colleagues in the range of 3.5 to $5.8 \mu \mathrm{g} / \mathrm{mL}$ in line with a lower caffeine dose used in our study (Lovallo et al., 1996, 2006). In their first study they had administered a single $3.3 \mathrm{mg} / \mathrm{kg}$ body weight dose of caffeine and in their second one either $250 \mathrm{mg}$ caffeine or placebo three times a day. Lovallo and colleagues (Lovallo et al., 1996) demonstrated that $3.3 \mathrm{mg} / \mathrm{kg}$ caffeine stimulated the pituitary-adrenal axis with increases in both ACTH and cortisol plasma concentrations. In their 2006 study the authors demonstrated similar changes in salivary cortisol (Lovallo et al., 2006). The mean salivary cortisone and cortisol levels found in our study were 
similar to those seen in the validation study of the salivary assay. For example, Mezzullo and colleagues (Mezzullo et al., 2016) reported mean morning salivary cortisone concentrations between $6.29 \pm 0.37 \mathrm{ng} / \mathrm{ml}$ and $13.7 \pm 1.02 \mathrm{ng} / \mathrm{ml}$ and afternoon concentrations between $5.82 \pm$ $0.36 \mathrm{ng} / \mathrm{ml}$ and $4.24 \pm 0.57 \mathrm{ng} / \mathrm{ml}$. Similar salivary cortisol concentrations were measured in the morning from $3.17 \pm 0.31 \mathrm{ng} / \mathrm{ml}$ to $0.82 \pm 0.01 \mathrm{ng} / \mathrm{ml}$, and in the afternoon from $0.68 \pm 0.01 \mathrm{ng} / \mathrm{ml}$ to $0.48 \pm 0.10 \mathrm{ng} / \mathrm{ml}$. Thus we could reproduce those measurements in both caffeine-naïve and caffeine consumers.

Our finding in caffeine-adapted consumers support a previous study which claimed that the intervention with a low dose of caffeine (i.e. $3 \mathrm{mg} / \mathrm{kg}$ body-weight) did not affect TMS measures such as motor thresholds, intra-cortical inhibition or facilitation, the cortical silent period and also the recruitment of pyramidal tract neurons (Orth et al., 2005). However, Orth and colleagues used a slightly longer poststimulation measurements (i.e. $45-60 \mathrm{~min}$ ), different route of caffeine intake (i.e. drinking coffee) and unspecified coffee consumption behaviour in their study population. This led to a difficult comparison with the present study. Another approach is to expand the poststimulation MEP measurements after one hour because we might miss caffeineinduced effects within 75 minutes after caffeine ingestions. This seems to be true because with the poststimulation measurements which less than one hour like in Orth and colleagues (Orth et al., 2005) also did not observe any changes in TMS measures. Another possible explanation other than dose is the variability in response to caffeine pharmacokinetics (Matthaei et al., 2016). However, there was no evidence to support the claim that changes in the concentrations of caffeine were associated with LTP/LTD-like plasticity induced by tACS and PAS 25 as shown in the previous studies (Hanajima et al., 2019; Zulkifly, Merkohitaj and Paulus, 2020). A possible explanation of contradicting results from caffeine consumers could be attributed to the behaviour of caffeine consumption. This remains speculative, as the earlier study did not prescribe the proper caffeine consumption behaviour for the caffeine consumers.

In both study groups, caffeine increased the poststimulation salivary concentrations of corticosteroids. The lack of correlations between poststimulation corticosteroid concentrations and poststimulation MEP measurements both in caffeine-naïve and caffeine-adapted consumers in contrast to caffeine influences may draw the attention to other influences of caffeine onto the brain. 
Since our finding does not confirm previous studies, which showed a link between cortisol levels and MEP amplitudes (Sale, Ridding and Nordstrom, 2008; Milani et al., 2010) the differences between morning and afternoon session results are an important variable to consider in plasticity studies beyond the influence of circulating corticosteroids. Both previous studies however used cortisol stimulation techniques, orally (Sale, Ridding and Nordstrom, 2008) and intravenously (Milani et al., 2010).

Changes in corticosteroid concentrations in our study were mainly due to the natural circadian rhythm and to the caffeine administration. An increase in cortisol levels is known when caffeine acts together with mental stress or physical exercise (Lovallo et al., 2006). In this present study, corticosteroid levels remained unaffected by stimulations also indicates that neither PAS nor tACS elicited a stress response; notably our stimulation protocols did not directly activate the hypothalamus-pituitary-adrenal axis. A previous study demonstrated an increase in serum cortisol levels after rTMS of the prefrontal cortex in a relaxed state (George et al., 1996). On the other hand, high-frequency rTMS does not affect the salivary cortisol in non-experimentally induced stress (Baeken, Rudi De Raedt, et al., 2009). Vice versa, one session of high-frequency rTMS attenuated the increase in salivary cortisol levels after an experimentally induced stress such as the Critical Feedback Task (CFT) in healthy females (Baeken et al., 2014). Anodal tDCS attenuated and cathodal tDCS augmented the salivary cortisol response after the Trier Social Stress Test (TSST) and after exposing the subjects with neutral images and those with emotionally negative content (Brunoni et al., 2013; Antal et al., 2014). Thus altogether stress responses seem to be variable.

Our study showed that in both study groups, men had higher corticosteroid levels than women in the afternoon sessions only. This was reflected in patterns in the changes in salivary cortisol concentrations. For instance, men responded more strongly to the mental stressors, while women responded strongly to food intake following exercise (Lovallo et al., 2006). Menstruation was not a confounding factor as we conducted both experiments after the menses had ended. This is consistent with a sex difference study of the hypothalamus-pituitary-adrenal axis response to a psychosocial stress test which demonstrated that salivary cortisol in men was similar to that in women in the luteal phase (Kirschbaum et al., 1999). A limitation of this study was the time point 
of the saliva collections. We may have missed some effects of corticosteroids on the effectiveness of NIBS because we did not collect the saliva immediately after the stimulation but after 30 minutes poststimulation measurements. An ideal approach would be to collect the saliva sample before the stimulation starts and repeatedly over the course of the poststimulation measurements. Cortisol plasma levels, e.g. peak at five and ten minutes after the injection of hydrocortisone, which is the equivalent of a pharmacologically simulated stress response (Milani et al., 2010). Our study was conducted in healthy university students. We did not control for factors such as exam stress and sleep deprivation, which might have affected the inter-individual and intra-day variability in cortisol levels. Both cortisol and academic stress may affect brain plasticity (Concerto, Patel, et al., 2017; Oster et al., 2017).

\subsection{CONCLUSIONS}

Caffeine intake increases salivary corticosteroid concentrations in caffeine-naïve and caffeineadapted persons. Prestimulation caffeine concentrations were associated with baseline cortical excitability in caffeine-adapted subjects but not with plastic aftereffects. The motor cortex of caffeine consumers is more excitable in the afternoon which may suggest that controlling the time

of day for plasticity induction studies may reduce variability in studies. No evidence of a relationship between poststimulation caffeine concentrations and poststimulation corticosteroids suggests that a moderate dose of $200 \mathrm{mg}$ caffeine does not influence poststimulation MEP measurements via the corticosteroid route and seems do not influence plasticity aftereffects. Higher salivary corticosteroid concentrations after caffeine administration in men reflects a differential response of gender to caffeine during the day. In our study, PAS and tACS did not elicit a HPA-axis response in either caffeine-naïve or caffeine-adapted participants.

\section{Conflict of interest}

M.F.M.Z, O.M., I.H., W.P., J.B declare no competing financial interest. 


\section{Funding}

MFMZ was supported by the Ministry of Education (MOE), Malaysia. This work was partly supported by the Göttingen Graduate Center for Neurosciences, Biophysics, and Molecular Biosciences (GGNB) of the Georg-August-Universität Göttingen.

\section{Acknowledgements}

We would like to thank Prof. Thomas Crozier for proofreading. 
Supplementary Table 4.1 Cortisone concentrations in saliva

\begin{tabular}{|c|c|c|c|}
\hline & Condition & $\begin{array}{c}\text { Cortisone concentration } \\
\text { PRE }(\mu \mathrm{g} / \mathrm{L})\end{array}$ & $\begin{array}{c}\text { Cortisone concentration } \\
\text { POST }(\mu \mathrm{g} / \mathrm{L})\end{array}$ \\
\hline \multirow{10}{*}{$\begin{array}{l}\text { Experiment } 1 \\
\text { (caffeine-naïve) }\end{array}$} & a) tACS & & \\
\hline & $1.0 \mathrm{~mA}$, placebo & $8.59(4.66-9.86)$ & $4.74(2.88-5.95)$ \\
\hline & $1.0 \mathrm{~mA}$, caffeine & $7.08(5.38-12.20)$ & $5.32(3.47-8.89)$ \\
\hline & $0.4 \mathrm{~mA}$, placebo & $7.64(4.94-12.46)$ & $4.68(3.52-5.73)$ \\
\hline & $0.4 \mathrm{~mA}$, caffeine & $7.48(4.78-11.08)$ & $5.11(4.00-9.13)$ \\
\hline & Sham, placebo & $7.77(6.67-12.10)$ & $3.73(2.86-6.90)$ \\
\hline & Sham, caffeine & $7.96(5.33-11.35)$ & $6.89(4.48-8.10)$ \\
\hline & b) PAS 25 & & \\
\hline & Placebo & $8.54(4.76-11.89)$ & $4.48(3.10-6.45)$ \\
\hline & Caffeine & $6.81(4.36-11.65)$ & $5.69(4.60-7.40)$ \\
\hline \multirow{10}{*}{$\begin{array}{l}\text { Experiment } 2 \\
\text { (caffeine consumers) }\end{array}$} & a) tACS $(1.0 \mathrm{~mA})$ & & \\
\hline & morning, placebo & $(5.28-13.80)$ & $5.14(3.71-7.06)$ \\
\hline & morning, caffeine & $10.08(6.36-12.45)$ & $5.19(3.86-6.91)$ \\
\hline & afternoon, placebo & $5.91(4.48-7.88)$ & $3.23(2.45-5.48)$ \\
\hline & afternoon, caffeine & $5.62(4.44-12.16)$ & $4.09(2.67-5.83)$ \\
\hline & b) tACS (Sham) & & \\
\hline & morning, placebo & $9.38(7.09-11.51)$ & $4.93(3.63-6.37)$ \\
\hline & morning, caffeine & $9.80(8.13-12.38)$ & $5.65(4.42-7.80)$ \\
\hline & afternoon, placebo & $6.68(4.42-10.08)$ & $3.58(2.49-5.94)$ \\
\hline & afternoon, caffeine & $6.49(4.26-9.12)$ & $4.21(2.81-5.49)$ \\
\hline
\end{tabular}

Values are given as median and interquantile range. $\mathrm{PRE}=$ before experiment; POST $=$ after experiment

Supplementary Table 4.2 Cortisol concentrations in saliva

\begin{tabular}{llcc}
\hline & Condition & $\begin{array}{c}\text { Cortisol concentration } \\
\text { PRE }(\boldsymbol{\mu g} / \mathbf{L})\end{array}$ & $\begin{array}{c}\text { Cortisol concentration } \\
\text { Post }(\boldsymbol{\mu g} / \mathbf{L})\end{array}$ \\
\hline $\begin{array}{l}\text { Experiment 1 } \\
\text { (caffeine-naïve) }\end{array}$ & a) tACS & $1.55(0.50-3.21)$ & $0.50(0.50-1.23)$ \\
& $1.0 \mathrm{~mA}$, placebo & $1.59(0.50-3.79)$ & $0.50(0.50-1.92)$ \\
& $1.0 \mathrm{~mA}$, caffeine & $1.33(0.50-4.06)$ & $0.50(0.50-1.01)$ \\
& $0.4 \mathrm{~mA}$, placebo & $1.36(0.50-4.25)$ & $0.50(0.50-1.86)$ \\
& $0.4 \mathrm{~mA}$, caffeine & $1.79(1.02-3.87)$ & $0.50(0.50-0.50)$ \\
& Sham, placebo & $2.08(0.50-4.08)$ & $0.50(0.50-1.78)$ \\
& Sham, caffeine & & $0.50(0.50-1.05)$ \\
& b) PAS 25 & $2.02(0.50-3.51)$ & $0.50(0.50-1.33)$ \\
& Placebo & $1.28(0.50-3.21)$ & \\
Cxperiment 2 & Caffeine & & $0.50(0.50-1.26)$ \\
& a) tACS (1.0 mA) & & $0.50(0.50-1.15)$ \\
& morning, placebo & $2.13(0.50-3.83)$ & $0.50(0.50-0.50)$ \\
& morning, caffeine & $2.18(0.50-3.70)$ & $0.50(0.50-0.50)$ \\
\hline
\end{tabular}




\begin{tabular}{lll} 
b) tACS $($ Sham $)$ & & \\
morning, placebo & $1.96(1.08-2.90)$ & $0.50(0.50-0.50)$ \\
morning, caffeine & $2.27(1.49-3.02)$ & $0.50(0.50-1.15)$ \\
afternoon, placebo & $0.50(0.50-1.89)$ & $0.50(0.50-0.50)$ \\
afternoon, caffeine & $0.50(0.50-1.16)$ & $0.50(0.50-0.50)$ \\
\hline
\end{tabular}

Values are given as median and interquantile range. $\mathrm{PRE}=$ before experiment; POST $=$ after experiment

Supplementary Table 4.3 Experiment 1: Results of the ANOVAs on salivary corticosteroids

\begin{tabular}{llllll}
\hline & Parameters & $\mathbf{d . f .}$ & $\mathbf{F}$ & $\mathbf{\mathbf { \eta } _ { \mathbf { p } } \mathbf { 2 }}$ & $\mathbf{p}$ \\
\hline 1) PAS 25 & Gender & 1,22 & 5.66 & 0.20 & $0.027^{*}$ \\
& Time & 1,22 & 18.47 & 0.46 & $<0.001^{*}$ \\
& Drug & 1,22 & 0.26 & 0.01 & 0.616 \\
& Time x Drug & 1,22 & 6.03 & 0.22 & $0.022^{*}$ \\
& Time x Drug x Gender & 1,22 & 5.58 & 0.20 & $0.027^{*}$ \\
& & & & \\
& Gender & 1,18 & 0.35 & 0.02 & 0.563 \\
& Time & 1,18 & 33.83 & 0.65 & $<0.001^{*}$ \\
& Stimulation & 2,36 & 0.82 & 0.04 & 0.449 \\
& Drug & 1,18 & 2.23 & 0.11 & 0.153 \\
& Time x Stimulation & 2,36 & 0.19 & 0.01 & 0.831 \\
& Time x Drug & 1,18 & 12.40 & 0.41 & $0.002^{*}$ \\
& Stimulation x Drug & 2,36 & 0.14 & 0.01 & 0.871 \\
& Time x Stimulation x Drug & 2,36 & 0.86 & 0.05 & 0.433 \\
& Time x Stimulation x Drug x & 2,36 & 4.83 & 0.21 & $0.014^{*}$ \\
& Gender & & & \\
\hline
\end{tabular}

* indicates $\mathrm{p}<0.05$. Abbreviations: d.f. $=$ degree of freedom; $\eta_{\mathrm{p}}{ }^{2}=$ partial eta square; $\mathrm{PAS}=$ paired associative stimulation; tACS $=$ transcranial alternating current stimulation.

Supplementary Table 4.4 Experiment 2: Results of ANOVAs on salivary corticosteroids

\begin{tabular}{lllll}
\hline Parameters & d.f. & $\mathbf{F}$ & $\mathbf{\eta}_{\mathbf{p}} \mathbf{2}^{2}$ & $\mathbf{p}$ \\
\hline Gender & 1,15 & 2.45 & 0.14 & 0.139 \\
Time & 1,15 & 51.75 & 0.78 & $<0.001^{*}$ \\
Stimulation & 1,15 & 1.58 & 0.10 & 0.228 \\
Day & 1,15 & 3.29 & 0.18 & 0.090 \\
Day x Gender & 1,15 & 8.70 & 0.37 & $0.010^{*}$ \\
Drug & 1,15 & 2.01 & 0.12 & 0.177 \\
Time x Stimulation & 1,15 & 0.88 & 0.06 & 0.362 \\
Time x Day & 1,15 & 0.11 & 0.01 & 0.747 \\
Time x Day x Gender & 1,15 & 4.96 & 0.25 & $0.042^{*}$ \\
Stimulation x Day & 1,15 & 0.02 & 0.00 & 0.891 \\
Time x Stimulation x Day & 1,15 & 0.10 & 0.01 & 0.760 \\
Time x Drug & 1,15 & 0.11 & 0.01 & 0.743 \\
\hline
\end{tabular}




\begin{tabular}{lllll}
\hline Stimulation x Drug & 1,15 & 2.80 & 0.16 & 0.115 \\
Time x Stimulation x Drug & 1,15 & 1.05 & 0.07 & 0.321 \\
Day x Drug & 1,15 & 2.92 & 0.16 & 0.108 \\
Time x Day x Drug & 1,15 & 0.36 & 0.02 & 0.558 \\
Stimulation x Day x Drug & 1,15 & 6.18 & 0.29 & $0.025^{*}$ \\
Time x Stimulation x Day x Drug & 1,15 & 0.74 & 0.05 & 0.403 \\
\hline
\end{tabular}

$*$ indicates $\mathrm{p}<0.05$. Abbreviations: d.f. $=$ degree of freedom; $\Pi_{\mathrm{p}}{ }^{2}=$ partial eta square.

a) Caffeine-naive

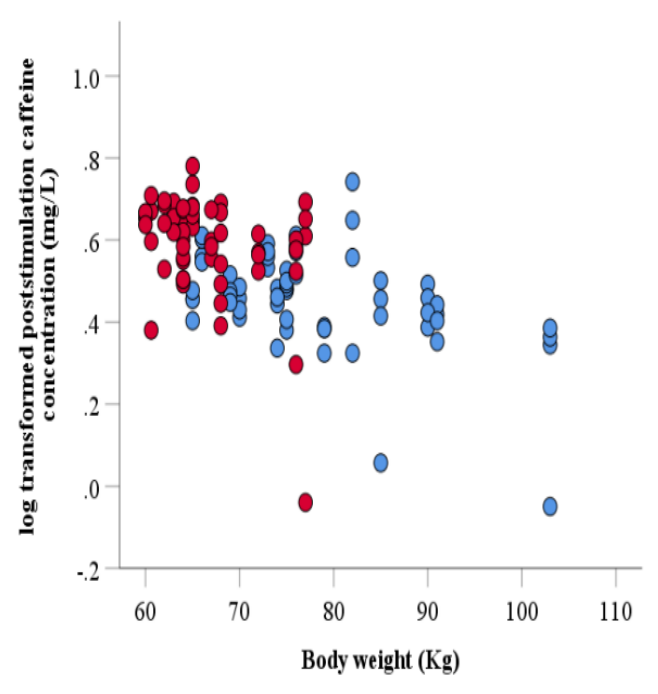

b) Caffeine-adapted consumers

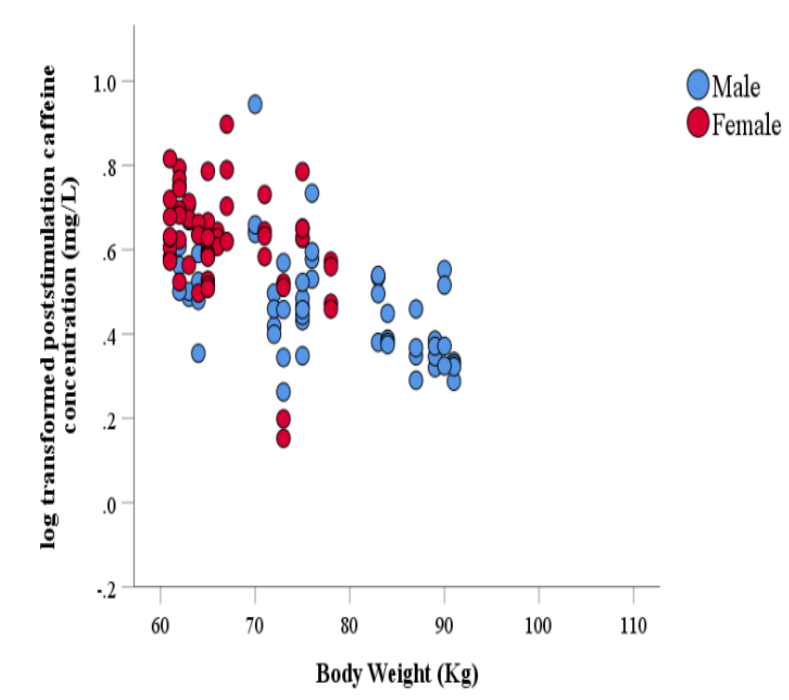

Supplementary Figure 4.1 Body weight and poststimulation caffeine concentrations. 
a) Caffeine-naive
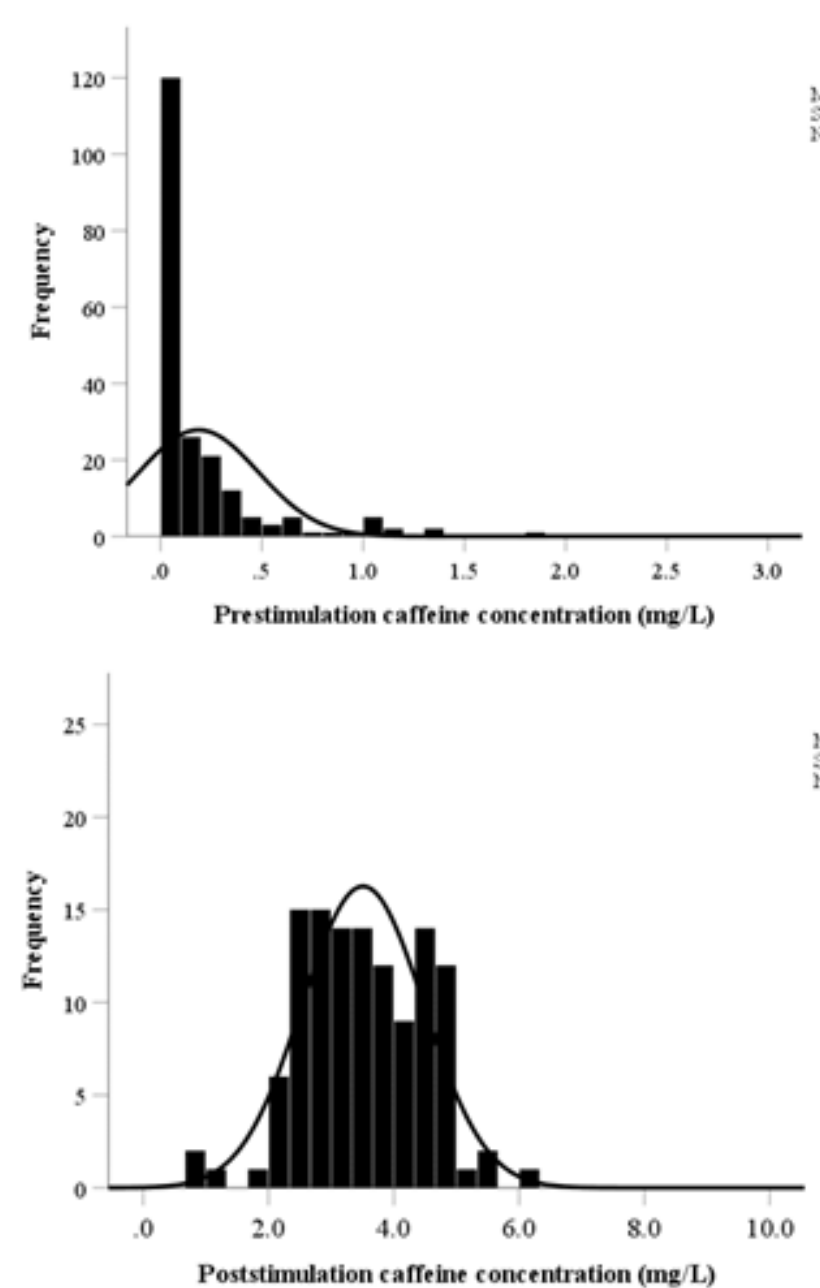

b) Caffeine-adapted consumers
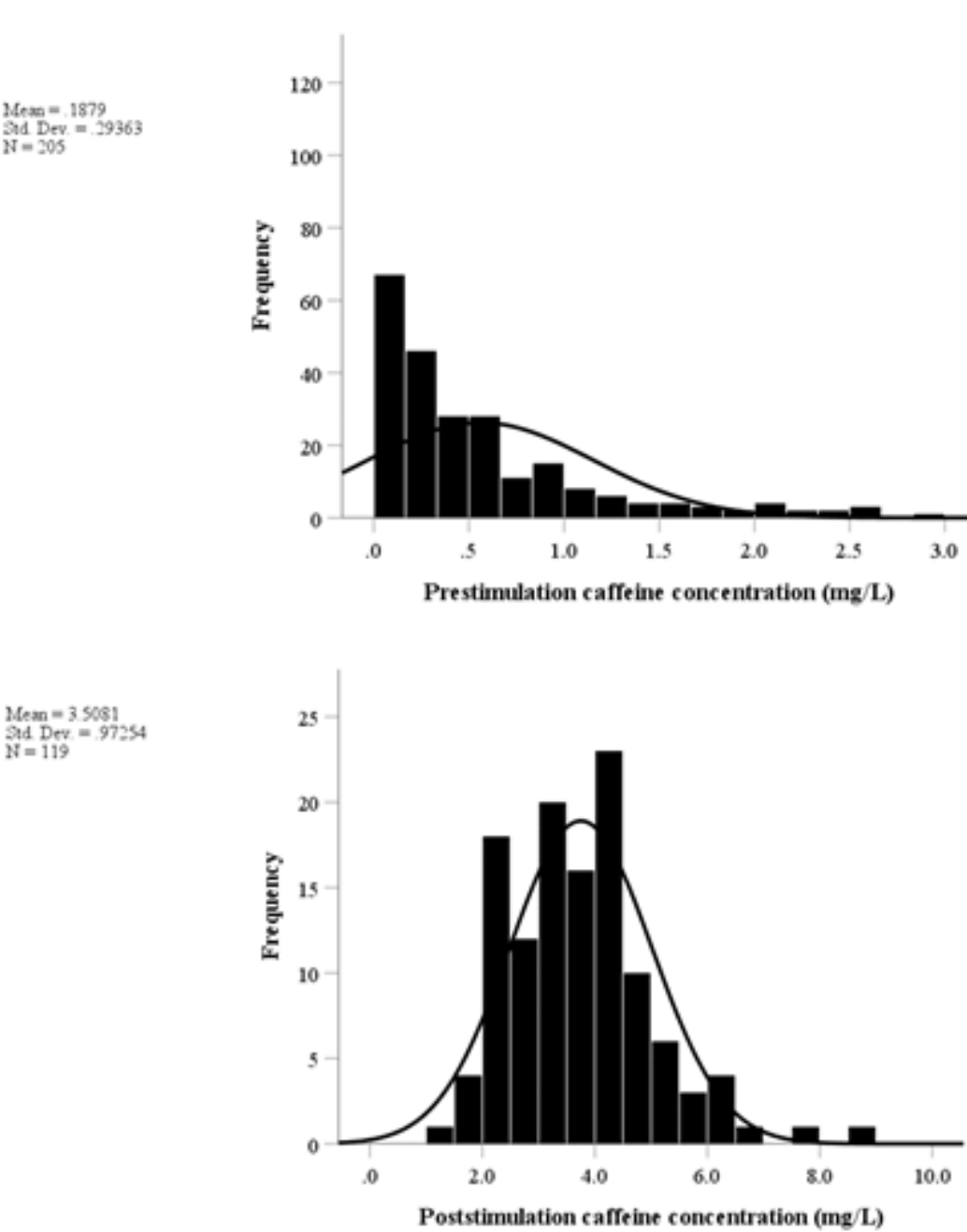

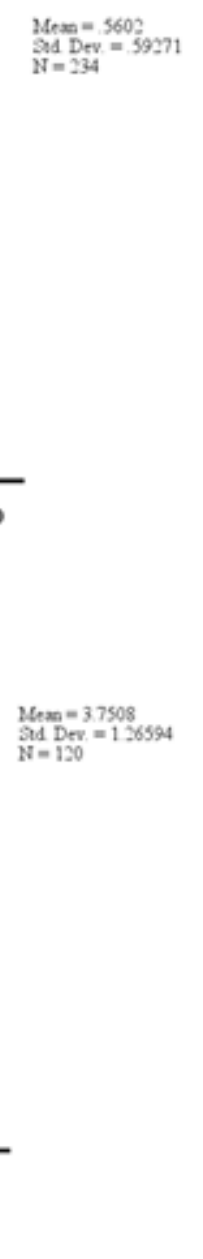

Supplementary Figure 4.2 Raw data of prestimulation and poststimulation caffeine concentrations. 


\section{CHAPTER V}

\section{GENERAL DISCUSSION AND CONCLUSION}

There is large inter-individual variability of the aftereffects induced by NIBS with less than half of the subject responding in an expected way to the stimulation (e.g. PAS: $39 \%$ - 52\%, TBS: $25 \%$ - 43\%, tDCS: 36\% - 45\% of responders) (Müller-Dahlhaus et al., 2008; Hamada et al., 2013; López-Alonso et al., 2014; Wiethoff, Hamada and Rothwell, 2014). This variability is also present in tACS studies. Similarly for tACS, previous studies have shown a modulatory effects of tACS on alpha-aftereffects (Zaehle, Rach and Herrmann, 2010; Neuling, Rach and Herrmann, 2013; Helfrich et al., 2014; Vossen, Gross and Thut, 2015). Subsequently, recently published studies reported null effects for tACS induced-aftereffects (Veniero et al., 2017; Clayton, Yeung and Kadosh, 2018; Fekete et al., 2018; Sliva et al., 2018; Stecher and Herrmann, 2018). The nonreproducibility issue is not new in the field and it is one of the major challenges to NIBS-induce plasticity studies, which hinders NIBS from being a therapeutic tool. In this thesis, we explored the possible known and unknown factors, which might contribute to variability in plasticity studies. In order to achieve this general aim of the thesis, we conducted a series of studies in the sensorimotor cortex and addressed possible internal determinants that affect plasticity aftereffects. The first three chapters (chapter 2, 3 and 4) include three separate studies in caffeine-naïve and caffeine-adapted subjects. Here, we explored modifiable factors, which include caffeine consumption, diurnal rhythm, alertness, stress hormones and ambient light on the plasticity aftereffects in the motor cortex using tACS and PAS. In this chapter, I will give a summary of the main findings of each experimental chapters and discuss the implications of the findings and future directions. At the end, I will give a general conclusion. 


\subsection{SUMMARY OF THE FINDINGS}

In CHAPTER 2, we examined the effects of espresso containing caffeine on the plasticity aftereffects of $140 \mathrm{~Hz}$ tACS in the motor cortex. Unexpectedly, we found a decrease in MEP facilitation induced by tACS after espresso with caffeine compared to tACS without caffeine. In addition, the facilitatory aftereffect of tACS was demolished after decaffeinated espresso consumption. This is an important finding, as it showed that (1) caffeine is only one of the confounding ingredients in coffee that impede the plasticity aftereffects, and (2) other bioactive compounds in decaffeinated espresso might underlie unobservable MEP facilitation of tACS. This suggests that the efficacy and reproducibility of tACS can be improved if the participants avoid both caffeine-containing and decaffeinated beverages before the experiment. We further investigated other factors which may influence neuroplasticity studies and NIBS responses. This includes caffeine intake, time of plasticity induction, alertness fluctuation during stimulation and ambient light. The key findings of this study were described in CHAPTER 3. We found that (1) caffeine strengthened and prolonged the MEP facilitation induced by PAS 25 in caffeine-naïve subjects and caffeine also enhanced the MEP size after $1 \mathrm{~mA}$ tACS both in caffeine-naïve and caffeine-adapted subjects. We showed that (2) the session in the morning has higher MEP amplitude after 1.0 mA tACS compared to sham. Also, (3) brain states during stimulation were associated with cortical excitability as shown by a positive relationship between alertness during stimulation and MEP amplitudes. In this study, we also addressed that (4) ambient light during experimental sessions affects motor cortical excitability. The outcomes from this study further support our finding in chapter 2; caffeine is the main confounder in plasticity induction studies. We also suggest that light exposure and alertness fluctuation are factors that reduce NIBS-induced plasticity. We took one step further to explore the influence of neurochemical factors on plasticity studies. In CHAPTER 4, we explored the relationships between salivary caffeine, corticosteroid concentrations and cortical excitability and plasticity aftereffects on the motor cortex. Also, we examined the effects of caffeine, stimulation type and time of day on the salivary corticosteroid concentrations. We showed that (1) increases in prestimulation caffeine concentrations were related to increases in the baseline MEPs for afternoon sessions in caffeine-adapted subjects, (2) in caffeine-naïve, higher poststimulation caffeine concentrations were associated with lower poststimulation MEPs after Sham, (3) poststimulation caffeine and poststimulation corticosteroid 
concentrations did not relate to plasticity aftereffects. In addition, we also found that (4) caffeine induces its effect by increasing the saliva corticosteroid concentrations in both caffeine-naïve and caffeine-adapted subjects, (5) PAS and tACS used in this study did not trigger the HPA-axis responses. In this study, we concluded that caffeine affects cortical excitability, but there was no association between salivary concentrations of poststimulation caffeine, poststimulation corticosteroids and plasticity induced aftereffects. A fixed experimental session is preferable due to changes of the corticosteroid activities over time.

A few molecular mechanisms can explain the robust effect of caffeine on neuroplasticity. First, as an adenosine receptor antagonist, caffeine inactivates the adenosine type 1 receptor (A1R) and hereby interferes with the inhibitory effects of adenosine on glutamatergic synaptic transmission (Kerkhofs et al., 2018). Next, an in vitro study demonstrated that caffeine inhibits $\gamma$ aminobutyric acid (GABA) release on the presynaptic site and suppresses GABAergic neuronal transmission at the post-synaptic neurons (Isokawa, 2016). Isokawa showed that this synaptic plasticity arises due to an increase of intracellular calcium $\left(\mathrm{Ca}^{2+}\right)$ and opening of the membrane channels (Isokawa, 2016). In an earlier study, theophylline, a group of adenosine receptor antagonist reduced the intracortical inhibition (ICI) of the human motor cortex (Nardone et al., 2004). This supports molecular findings as described above that caffeine induces its effects by inhibiting GABAergic transmission (Nardone et al., 2004). A stronger and prolonged plasticity effect of caffeine after PAS 25 in our study confirms the hypothesis that $\mathrm{Ca}^{2+}$ modulates the directions and magnitude of plasticity induced by NIBS studies. Both NMDA receptors and $\mathrm{Ca}^{2+}$ channels play an important role in PAS-induced plasticity (Stefan et al., 2002; Weise et al., 2017). As a synapse-specific protocol targeting only those cells being preactivated by somatosensory stimulation of the median nerve (Nitsche et al., 2007), PAS in combination with caffeine probably further increases the intracellular $\mathrm{Ca}^{2+}$ concentrations and leads to stronger synaptic transmission. On the other hand, tACS influences all cells in the electric field and can thus be classified as a synapse-unspecific method similar to rTMS and tDCS protocols (Nitsche et al., 2007). This may explain smaller aftereffects induced by tACS in combination with caffeine compared to the aftereffects with PAS. Caffeine exerted its effects by also increasing salivary corticosteroid concentrations. This indicates that $200 \mathrm{mg}$ caffeine dose is sufficient to modulate the HPA-axis. In an animal study, a dose of $2 \mathrm{mg} / \mathrm{kg}$ elevates plasma corticosterone and adrenocorticotrophic 
hormone (ACTH) levels 30 minutes after injection of caffeine, and a dose of $30 \mathrm{mg} / \mathrm{kg}$ sustains the plasma hormone levels elevation up to 120 minutes (Patz et al., 2006).

Our findings confirm the role of alertness during tACS stimulation on cortical excitability. It is consistent with the earlier study by Stefan and colleagues which shows that shifting away subject's attention or conflicting their attention with cognitive tasks during stimulation diminished the LTP-like plasticity effects induced by PAS in the motor cortex (Stefan, Wycislo and Classen, 2004). An important implication of our finding in clinical practice is that patients who have an intact attention-control system may benefit from motor performance or plasticity inductions more than patients with an impaired attention-control system. Rinne and colleagues showed that impairment in attention-control in stroke patients is associated with lower motor dexterity (Rinne et al., 2018). We found no correlation between the PUI and MEP size for the caffeine conditions and for sham/placebo stimulation. Only $1 \mathrm{~mA}$ tACS with placebo drug showed a reverse correlation, thus higher MEPs in more alert subjects. Thus this does not support the theory by Paulus and Rothwell (Paulus and Rothwell, 2016), which claims that attention should activate neurons and may reduce stimulation efficacy by "leaky membranes". If this theory would be true, we should observe an excitability decrease in case of higher attention during stimulation, which was not the case.

Consistent studies from TMS and neuroimaging experiments show that light deprivation increases visual cortex excitability as indicated by reduced phosphenes threshold and increase in BOLD signals (Boroojerdi et al., 2000, 2001; Fierro et al., 2005; Pitskel et al., 2007; de Graaf et al., 2017). Our study showed that short-term light deprivation (i.e. 10 minutes) reduced motor cortical excitability. In the first place, it seems to contradict previous studies mentioned above. But, a recent study showed a reduction in the P60 component evoked by TMS (so-called transcranial evoked potential (TEP)) after dark adaptation in the visual cortex (Zazio et al., 2019). P60 and N100 components were also reduced when evoked directly at the motor cortex under dark adaptation (Casula et al., 2014). Strigaro and colleagues confirm a connection between visual and motor cortex as shown by MEP amplitude changes $18-40 \mathrm{~ms}$ after a TMS pulses being applied on the occipital region (Strigaro et al., 2015). Short-interval intracortical inhibition (SICI), a marker of short-lasting postsynaptic inhibition (Ziemann et al., 2015), was increased in the motor 
cortex after stimulation of the visual cortex. MEP suppression indicates that excitatory synapses in the visual cortex affect the inhibitory circuit in M1 (Strigaro et al., 2015). This confirms the modulatory effects of light deprivation on the motor and visual cortex excitability.

Another important finding is the effect of circadian rhythms on cortical excitability. We found that the MEP suppression due to light deprivation was prevented after applying tACS in the morning but not in the afternoon and not after sham tACS. This shows that tACS- induced facilitatory effects are stronger in the morning sessions. Other excitability parameters such as longer cortical silent period (CSP) duration and longer long-interval intracortical inhibition (LICI) were higher in the morning as compared to the afternoon (Sale, Ridding and Nordstrom, 2008; Lang et al., 2011). Other parameters like motor thresholds (MT), $1 \mathrm{mV}$ MEP and intracortical facilitation (ICF) were unchanged (Lang et al., 2011). PAS was published to be higher in the evening than in the morning (Sale, Ridding and Nordstrom, 2008). However, these studies differ in several aspects. We recruited caffeine-adapted subjects which had lower MT (i.e. resting MT (RMT) and $1 \mathrm{mV}$ peak-to-peak amplitude $\mathrm{MT}_{1 \mathrm{mV}}$ ) compared to caffeine-naïve subjects. MT is an index of axonal excitability (Ziemann et al., 2015), and a lower motor threshold in our study indicates that the corticospinal system among our caffeine-adapted subjects was more excitable compared to caffeine-naïve subjects.

In the afternoon sessions; elevated prestimulation caffeine concentrations were associated with higher baseline MEP amplitudes whereas tACS induced aftereffects were not correlated. This indicates that baseline cortical excitability was more pronounced in the afternoon. Thus, our result suggest that experimental sessions in the morning requires higher stimulation intensity to induce

plasticity aftereffects due to low baseline excitability thresholds. Further circadian factors may play a role (Kuhn et al., 2016).

\subsection{IMPLICATIONS AND FUTURE DIRECTIONS}

Some insights from the studies presented in this thesis have its own scientific, clinical and methodological implications. First, in the context of neuroplasticity and NIBS efficacy, caffeine is not only a confounding factor which enhances the plasticity aftereffects on the sensorimotor 
cortex, but it also affects the HPA axis by modulating circulating salivary corticosteroid concentrations. Different dose of caffeine concentration may change the directions and magnitude of NIBS-induced plasticity as we showed that espresso with caffeine diminished tACS- induced facilitatory effetcs whereas higher dosage (i.e. $200 \mathrm{mg}$ caffeine table) enhanced and prolonged LTP-like plasticity of PAS 25. Caffeine consumption behaviours also influence the neuroplasticity aftereffects as demonstrated by differences in motor thresholds and in the directions of circulating corticosteroid concentrations with cortical plasticity in caffeine-naive and caffeine-adapted subjects. Caffeine intake should be documented and controlled in future studies to improve the efficacy and optimize the reproducibility of NIBS. Second, in the context of study design, fluctuation of alertness during stimulation, light exposure in the laboratory and a fixed time of experimental sessions should be taken into account in order to optimize the stimulation outcomes and reduce intra- and inter-individual variability of NIBS-induced plasticity. In addition, an important finding of this study for clinical practice is to differentiate which patient will gain more benefits from neurorehabilitation interventions. Also, the nature of compensatory changes in case of damage to cortical regions affecting other parts of the cortex in its networks has to be taken into account. We showed that cortical excitability changes in visual cortex (i.e. light deprivation) modulate excitability in the motor region. This may explain neuroplasticity among patients with neurological disorders such as stroke, traumatic brain injury and vision loss.

Ongoing work is on the way to reduce the variability and improve the efficacy of NIBS. The outcomes in this study joined a previous background of knowledge to address intra- and interindividual variability on NIBS responses. Brain states such as physical activity and alertness fluctuation should be controlled and monitored during experiments. One approach is to use priming stimulation to 'normalize' the brain state before the NIBS intervention (Ziemann and Siebner, 2015). Another approach is to combine NIBS with neuroimaging technique such as highdensity EEG. Recording the brain states during the NIBS offer a great potential to modulate, entrain or interfere brain oscillation at the optimal power or phase to induce excitability. In the motor system, MEP is a common readout as an index of cortical excitability. Its excitability measure indicates the activation of specific neurons that control the target muscle. However, it is highly variable and easily affected by muscle contractions and also fluctuation in the cortico-spinal excitability (Hordacre et al., 2017). EEG measurements can reveal neuronal activity, and previous 
studies showed an inverse relationship between alpha power and firing rate or cortical excitability in sensorimotor regions (Zarkowski et al., 2006; Sauseng et al., 2009; Haegens et al., 2011). In later studies, spontaneous alpha and beta-band power was shown to predict TMS-induced MEP size (Mäki and Ilmoniemi, 2010; Schulz et al., 2014). We conclude that we can utilize tACS technique to entrain alpha and beta power in order to stabilize the motor system. At this point, a promising meta-analysis demonstrated that beta-tACS at the stimulation intensity above $1 \mathrm{~mA}$ showed a robust increase in M1 (Wischnewski, Schutter and Nitsche, 2019). This is potentially useful for monitoring and controlling brain states during stimulation (i.e. closed-loop stimulation) using neuroimaging techniques (i.e. EEG/MEG), which is one approach to deal with fluctuation in brain activity (Feurra et al., 2013; Zrenner et al., 2016, 2018; Thut et al., 2017; Bergmann, 2018).

\subsection{CONCLUSION}

In this thesis we demonstrated caffeine to be an important confounding factors to NIBS- induced plasticity. Caffeine, which is widely used psychostimulant, not only enhances cortical excitability but also modulates HPA axis by increasing the concentrations of circulating salivary poststimulation corticosteroid. Apart from that, this thesis addresses alertness fluctuation during stimulation, light exposure and diurnal rhythms as sources of intra- and inter-individual variability, which reduce the effectiveness of NIBS and plasticity inductions. Caffeine consumption behaviour also determines NIBS responses. All of these confounding factors are modifiable and controllable. Thus future studies need to implement a strict rule by documenting and optimizing them to reduce variability and enhance the efficacy of NIBS. 


\section{BIBLIOGRAPHY}

Abellaneda-Pérez, K. et al. (2020) 'Differential tDCS and tACS Effects on Working MemoryRelated Neural Activity and Resting-State Connectivity', Frontiers in Neuroscience, 13. doi: 10.3389/fnins.2019.01440.

Alekseichuk, I. et al. (2016) 'Spatial Working Memory in Humans Depends on Theta and High Gamma Synchronization in the Prefrontal Cortex', Current Biology, 26(12), pp. 15131521. doi: 10.1016/j.cub.2016.04.035.

Ambrus, G. G. et al. (2015) 'Bi-frontal transcranial alternating current stimulation in the ripple range reduced overnight forgetting', Frontiers in Cellular Neuroscience, 9, pp. 1-7. doi: 10.3389/fncel.2015.00374.

Angelakis, E. et al. (2013) 'Transcranial alternating current stimulation reduces symptoms in intractable idiopathic cervical dystonia: A case study', Neuroscience Letters. Elsevier Ireland Ltd, 533(1), pp. 39-43. doi: 10.1016/j.neulet.2012.11.007.

Antal, A. et al. (2007) 'Towards unravelling task-related modulations of neuroplastic changes induced in the human motor cortex', European Journal of Neuroscience, 26(9), pp. 26872691. doi: 10.1111/j.1460-9568.2007.05896.x.

Antal, A. et al. (2008) 'Comparatively weak after-effects of transcranial alternating current stimulation (tACS) on cortical excitability in humans', Brain Stimulation, 1(2), pp. 97105. doi: 10.1016/j.brs.2007.10.001.

Antal, A. et al. (2010) 'Brain-derived neurotrophic factor (BDNF) gene polymorphisms shape cortical plasticity in humans', Brain Stimulation. Elsevier Inc, 3(4), pp. 230-237. doi: 10.1016/j.brs.2009.12.003.

Antal, A. et al. (2014) 'Transcranial electrical stimulation modifies the neuronal response to psychosocial stress exposure', Human Brain Mapping, 35(8), pp. 3750-3759. doi: 10.1002/hbm.22434.

Antal, A. et al. (2017) 'Low intensity transcranial electric stimulation: Safety, ethical, legal regulatory and application guidelines', Clinical Neurophysiology, 128(9), pp. 1774-1809. doi: 10.1016/j.clinph.2017.06.001.

Antal, A., Ambrus, G. G. and Chaieb, L. (2014) 'Toward unraveling reading-related modulations of tDCS-induced neuroplasticity in the human visual cortex', Frontiers in Psychology. Frontiers Research Foundation, 5, p. 642. doi: 10.3389/fpsyg.2014.00642.

Antal, A. and Herrmann, C. S. (2016) 'Transcranial Alternating Current and Random Noise Stimulation: Possible Mechanisms', Neural Plasticity. Hindawi Publishing Corporation, 2016, pp. 1-12. doi: 10.1155/2016/3616807. 
Antal, A. and Paulus, W. (2013) 'Transcranial alternating current stimulation (tACS)', Frontiers in Human Neuroscience, 7, pp. 1-4. doi: 10.3389/fnhum.2013.00317.

Bae, Y. J. et al. (2019) 'Salivary cortisone, as a biomarker for psychosocial stress, is associated with state anxiety and heart rate', Psychoneuroendocrinology, 101, pp. 35-41. doi: 10.1016/j.psyneuen.2018.10.015.

Baeken, C., De Raedt, R., et al. (2009) 'The impact of one HF-rTMS session on mood and salivary cortisol in treatment resistant unipolar melancholic depressed patients', Journal of Affective Disorders. Elsevier B.V., 113(1-2), pp. 100-108. doi: 10.1016/j.jad.2008.05.008.

Baeken, C., De Raedt, Rudi, et al. (2009) 'The impact of one session of HF-rTMS on salivary cortisol in healthy female subjects', The World Journal of Biological Psychiatry, 10(4-2), pp. 586-590. doi: 10.1080/15622970701560351.

Baeken, C. et al. (2014) 'One left dorsolateral prefrontal cortical HF-rTMS session attenuates HPA-system sensitivity to critical feedback in healthy females', Neuropsychologia. Elsevier Ltd, 57(1), pp. 112-121. doi: 10.1016/j.neuropsychologia.2014.02.019.

Bekar, L. et al. (2008) 'Adenosine is crucial for deep brain stimulation-mediated attenuation of tremor', Nature Medicine, 14(1), pp. 75-80. doi: 10.1038/nm1693.

Bennett, M. C. et al. (1991) 'Serum corticosterone level predicts the magnitude of hippocampal primed burst potentiation and depression in urethane-anesthetized rats', Psychobiology, 19(4), pp. 301-307. doi: https://doi.org/10.3758/BF03332083.

Bergmann, T. O. (2018) 'Brain state-dependent brain stimulation', Frontiers in Psychology, 9, p. 2108. doi: 10.3389/fpsyg.2018.02108.

Biabani, M. et al. (2018) 'The minimal number of TMS trials required for the reliable assessment of corticospinal excitability, short interval intracortical inhibition, and intracortical facilitation', Neuroscience Letters. Elsevier, 674, pp. 94-100. doi: 10.1016/j.neulet.2018.03.026.

Bikson, M. et al. (2016) 'Safety of Transcranial Direct Current Stimulation: Evidence Based Update 2016', Brain Stimulation. Elsevier Inc., 9(5), pp. 641-661. doi: 10.1016/j.brs.2016.06.004.

Blaise, J. H. et al. (2018) 'Caffeine consumption disrupts hippocampal long-term potentiation in freely behaving rats', Physiological Reports, 6(5), pp. 1-8. doi: 10.14814/phy2.13632.

Boroojerdi, B. et al. (2000) 'Enhanced excitability of the human visual cortex induced by shortterm light deprivation', Cerebral Cortex, 10, pp. 529-534.

Boroojerdi, B. et al. (2001) 'Mechanisms underlying rapid experience-dependent plasticity in the human visual cortex', Proceedings of the National Academy of Sciences of the United States of America, 98(25), pp. 14698-14701. doi: 10.1073/pnas.251357198. 
Brunoni, A. R. et al. (2013) 'Polarity- and valence-dependent effects of prefrontal transcranial direct current stimulation on heart rate variability and salivary cortisol', Psychoneuroendocrinology, 38(1), pp. 58-66. doi: 10.1016/j.psyneuen.2012.04.020.

Caipa, A., Alomar, M. and Bashir, S. (2018) 'TMS as a tool to investigate the effect of pharmacological medications on cortical plasticity', European Review for Medical and Pharmacological Sciences, 22(3), pp. 844-852. doi: 10.26355/eurrev_201802_14321.

Calker, D. et al. (2019) 'The role of adenosine receptors in mood and anxiety disorders', Journal of Neurochemistry, 151(1), pp. 11-27. doi: 10.1111/jnc.14841.

Calker, D. van, Müller, M. and Hamprecht, B. (1979) 'Adenosine Regulates Via Two Different Types of Receptors, the Accumulation of Cyclic Amp in Cultured Brain Cells', Journal of Neurochemistry, 33(5), pp. 999-1005. doi: 10.1111/j.1471-4159.1979.tb05236.x.

Cambieri, C. et al. (2017) 'Effects of visual deprivation on primary motor cortex excitability: a study on healthy subjects based on repetitive transcranial magnetic stimulation', Experimental Brain Research. Springer Berlin Heidelberg, 235(7), pp. 2059-2067. doi: 10.1007/s00221-017-4945-0.

Carson, R. G. and Kennedy, N. C. (2013) 'Modulation of human corticospinal excitability by paired associative stimulation', Frontiers in Human Neuroscience. Frontiers, 7(823), pp. 1-28. doi: 10.3389/fnhum.2013.00823.

Casula, E. P. et al. (2014) 'Low-frequency rTMS inhibitory effects in the primary motor cortex: Insights from TMS-evoked potentials', NeuroImage. Academic Press Inc., 98, pp. 225232. doi: 10.1016/j.neuroimage.2014.04.065.

Cerqueira, V. et al. (2006) 'Does caffeine modify corticomotor excitability?', Neurophysiologie Clinique, 36(4), pp. 219-226. doi: 10.1016/j.neucli.2006.08.005.

Chaieb, L. et al. (2014) 'Brain-derived neurotrophic factor: Its impact upon neuroplasticity and neuroplasticity inducing transcranial brain stimulation protocols', Neurogenetics, 15(1), pp. 1-11. doi: 10.1007/s10048-014-0393-1.

Chaieb, L., Antal, A. and Paulus, W. (2011) 'Transcranial alternating current stimulation in the low $\mathrm{kHz}$ range increases motor cortex excitability', Restorative Neurology and Neuroscience, 29(3), pp. 167-175. doi: 10.3233/RNN-2011-0589.

Cheeran, B. et al. (2008) 'A common polymorphism in the brain-derived neurotrophic factor gene ( BDNF) modulates human cortical plasticity and the response to rTMS', Journal of Physiology, 586(23), pp. 5717-5725. doi: 10.1113/jphysiol.2008.159905.

Chen, K. H. and Huang, Y. Z. (2018) 'The change of motor cortical excitability between eyes open and closed conditions', NeuroReport, 29(3), pp. 214-218. doi: 10.1097/WNR.0000000000000955. 
Cirillo, G. et al. (2017) 'Neurobiological after-effects of non-invasive brain stimulation', Brain Stimulation. Elsevier Ltd, 10(1), pp. 1-18. doi: 10.1016/j.brs.2016.11.009.

Clayton, M. S., Yeung, N. and Kadosh, R. C. (2018) 'The effects of $10 \mathrm{~Hz}$ transcranial alternating current stimulation on audiovisual task switching', Frontiers in Neuroscience, 12(FEB), p. 67. doi: 10.3389/fnins.2018.00067.

Concerto, C., Patel, D., et al. (2017) 'Academic stress disrupts cortical plasticity in graduate students', Stress. Informa UK Ltd., 20(2), pp. 212-216. doi: 10.1080/10253890.2017.1301424.

Concerto, C., Infortuna, C., et al. (2017) 'Caffeinated energy drink intake modulates motor circuits at rest, before and after a movement', Physiology and Behavior. Elsevier, 179, pp. 361368. doi: 10.1016/j.physbeh.2017.07.013.

Conde, V. et al. (2012a) 'Cortical thickness in primary sensorimotor cortex influences the effectiveness of paired associative stimulation', NeuroImage. Elsevier Inc., 60(2), pp. 864870. doi: 10.1016/j.neuroimage.2012.01.052.

Conde, V. et al. (2012b) 'Cortical thickness in primary sensorimotor cortex influences the effectiveness of paired associative stimulation', NeuroImage, 60(2), pp. 864-870. doi: 10.1016/j.neuroimage.2012.01.052.

Conte, A. et al. (2007) 'Attention influences the excitability of cortical motor areas in healthy humans’, Experimental Brain Research, 182(1), pp. 109-117. doi: 10.1007/s00221-0070975-3.

Conte, A. et al. (2008) 'Effects of attention on inhibitory and facilitatory phenomena elicited by paired-pulse transcranial magnetic stimulation in healthy subjects', Experimental Brain Research, 186(3), pp. 393-399. doi: 10.1007/s00221-007-1244-1.

Corp, D. T. et al. (2020) 'Large-scale analysis of interindividual variability in theta-burst stimulation data: Results from the "Big TMS Data Collaboration"', Brain Stimulation, 13(5), pp. 1476-1488. doi: 10.1016/j.brs.2020.07.018.

Daguet, I., Bouhassira, D. and Gronfier, C. (2019) 'Baseline Pupil Diameter Is Not a Reliable Biomarker of Subjective Sleepiness', Frontiers in Neurology, 10(108), pp. 1-11. doi: 10.3389/fneur.2019.00108.

Diepen, R. M. Van, Foxe, J. J. and Mazaheri, A. (2019) 'The functional role of alpha-band activity in attentional processing : the current zeitgeist and future outlook', Current Opinion in Psychology. Elsevier Ltd, 29, pp. 229-238. doi: 10.1016/j.copsyc.2019.03.015.

Dinse, H. R. et al. (2017) 'The stress hormone cortisol blocks perceptual learning in humans', Psychoneuroendocrinology. Elsevier Ltd, 77, pp. 63-67. doi: 10.1016/j.psyneuen.2016.12.002. 
Duncan, M. J. and Oxford, S. W. (2011) 'The effect of caffeine ingestion on mood state and bench press performance to failure', Journal of Strength and Conditioning Research, 25(1), pp. 178-185. doi: 10.1519/JSC.0b013e318201bddb.

Eggert, T. et al. (2012) 'The pupillographic sleepiness test in adults: Effect of age, gender, and time of day on pupillometric variables', American Journal of Human Biology, 24(6), pp. 820-828. doi: 10.1002/ajhb.22326.

Fekete, T. et al. (2018) 'Multi-electrode alpha tACS during varying background tasks fails to modulate subsequent alpha power', Frontiers in Neuroscience, 12(JUN). doi: 10.3389/fnins.2018.00428.

Ferreira, D. D. P. et al. (2014) 'Caffeine potentiates the release of GABA mediated by NMDA receptor activation: Involvement of A1adenosine receptors', Neuroscience, 281, pp. 208215. doi: 10.1016/j.neuroscience.2014.09.060.

Feurra, M. et al. (2011) 'Frequency-Dependent Tuning of the Human Motor System Induced by Transcranial Oscillatory Potentials', Journal of Neuroscience, 31(34), pp. 12165-12170. doi: 10.1523/JNEUROSCI.0978-11.2011.

Feurra, M. et al. (2013) 'State-dependent effects of transcranial oscillatory currents on the motor system: What you think matters', Journal of Neuroscience, 33(44), pp. 17483-17489. doi: 10.1523/JNEUROSCI.1414-13.2013.

Fierro, B. et al. (2005) 'Modulatory effects of low- and high-frequency repetitive transcranial magnetic stimulation on visual cortex of healthy subjects undergoing light deprivation', Journal of Physiology. John Wiley \& Sons, Ltd, 565(2), pp. 659-665. doi: 10.1113/jphysiol.2004.080184.

Freitas, C., Farzan, F. and Pascual-Leone, A. (2013) 'Assessing brain plasticity across the lifespan with transcranial magnetic stimulation: why, how, and what is the ultimate goal?', Frontiers in Neuroscience, 7(42), pp. 1-17. doi: 10.3389/fnins.2013.00042.

Fries, P. (2001) 'Modulation of Oscillatory Neuronal Synchronization by Selective Visual Attention', Science, 291(5508), pp. 1560-1563. doi: 10.1126/science.1055465.

George, M. S. et al. (1996) 'Changes in mood and hormone levels after rapid-rate transcranial magnetic stimulation (rTMS) of the prefrontal cortex', The Journal of Neuropsychiatry and Clinical Neurosciences, 8(2), pp. 172-180. doi: 10.1176/jnp.8.2.172.

de Goede, A. A., ter Braack, E. M. and van Putten, M. J. A. M. (2018) 'Accurate Coil Positioning is Important for Single and Paired Pulse TMS on the Subject Level', Brain Topography. Springer US, 31(6), pp. 917-930. doi: 10.1007/s 10548-018-0655-6.

Goldsworthy, M. R. et al. (2014) 'Inter-subject variability of LTD-like plasticity in human motor cortex: A matter of preceding motor activation', Brain Stimulation. Elsevier Ltd, 7(6), pp. 864-870. doi: 10.1016/j.brs.2014.08.004. 
de Graaf, T. A. et al. (2017) 'Seeing in the dark: Phosphene thresholds with eyes open versus closed in the absence of visual inputs', Brain Stimulation. Elsevier Ltd, 10(4), pp. 828835. doi: 10.1016/j.brs.2017.04.127.

Graupner, M. and Brunel, N. (2012) 'Calcium-based plasticity model explains sensitivity of synaptic changes to spike pattern, rate, and dendritic location', Proceedings of the National Academy of Sciences of the United States of America, 109(10), pp. 3991-3996. doi: 10.1073/pnas.1109359109.

Grundey, J. et al. (2018) 'Nicotine modulates human brain plasticity via calcium-dependent mechanisms', Journal of Physiology, 596(22), pp. 5429-5441. doi: 10.1113/JP276502.

Guerra, A. et al. (2020) 'Solutions for managing variability in non-invasive brain stimulation studies', Neuroscience Letters. Elsevier Ireland Ltd, 719, p. 133332. doi: 10.1016/j.neulet.2017.12.060.

Gundlach, C. et al. (2017) 'Modulation of Somatosensory Alpha Rhythm by Transcranial Alternating Current Stimulation at Mu-Frequency', Frontiers in Human Neuroscience, 11(August), pp. 1-11. doi: 10.3389/fnhum.2017.00432.

Gundlach, C. et al. (2020) 'Reduction of somatosensory functional connectivity by transcranial alternating current stimulation at endogenous mu-frequency', NeuroImage. Academic Press Inc., 221, p. 117175. doi: 10.1016/j.neuroimage.2020.117175.

Haegens, S. et al. (2011) ' $\alpha$-Oscillations in the monkey sensorimotor network influence discrimination performance by rhythmical inhibition of neuronal spiking', Proceedings of the National Academy of Sciences of the United States of America, 108(48), pp. 1937719382. doi: 10.1073/pnas.1117190108.

Haegens, S., Händel, B. F. and Jensen, O. (2011) 'Top-down controlled alpha band activity in somatosensory areas determines behavioral performance in a discrimination task', Journal of Neuroscience, 31(14), pp. 5197-5204. doi: 10.1523/JNEUROSCI.5199-10.2011.

Hakamata, Y. et al. (2019) 'Cortisol-related hippocampal-extrastriate functional connectivity explains the adverse effect of cortisol on visuospatial retrieval', Psychoneuroendocrinology. Elsevier, 109(April), p. 104310. doi: 10.1016/j.psyneuen.2019.04.013.

Hallett, M. (2007) 'Transcranial Magnetic Stimulation: A Primer', Neuron, 55(2), pp. 187-199. doi: 10.1016/j.neuron.2007.06.026.

Hamada, M. et al. (2013) 'The role of interneuron networks in driving human motor cortical plasticity', Cerebral Cortex, 23(7), pp. 1593-1605. doi: 10.1093/cercor/bhs 147.

Hanajima, R. et al. (2019) 'Effect of caffeine on long-term potentiation-like effects induced by quadripulse transcranial magnetic stimulation', Experimental Brain Research. Springer Berlin Heidelberg, 237(3), pp. 647-651. doi: 10.1007/s00221-018-5450-9. 
Hannah, R., Iacovou, A. and Rothwell, J. C. (2019) 'Direction of TDCS current flow in human sensorimotor cortex influences behavioural learning', Brain Stimulation, 12(3), pp. 684692. doi: 10.1016/j.brs.2019.01.016.

Hebb, D. O. (1949) 'The Organisation of Behavior', in New York: Wiley and Sons, pp. 1-365. doi: 10.1364/OL.24.000954.

Hedges, D. W. et al. (2002) 'Transcranial magnetic stimulation (TMS) effects on testosterone, prolactin, and corticosterone in adult male rats', Biological Psychiatry, 51(5), pp. 417-421. doi: 10.1016/S0006-3223(01)01266-5.

Helfrich, R. F. et al. (2014) 'Entrainment of brain oscillations by transcranial alternating current stimulation.', Current biology : CB, 24(3), pp. 333-9. doi: 10.1016/j.cub.2013.12.041.

Herrmann, C. S. et al. (2013) 'Transcranial alternating current stimulation: a review of the underlying mechanisms and modulation of cognitive processes', Frontiers in Human Neuroscience, 7, pp. 1-13. doi: 10.3389/fnhum.2013.00279.

Hordacre, B. et al. (2017) 'Variability in neural excitability and plasticity induction in the human cortex: A brain stimulation study’, Brain Stimulation. Elsevier Ltd, 10(3), pp. 588-595. doi: 10.1016/j.brs.2016.12.001.

$\mathrm{Hu}$, Y. et al. (2019) 'Paired associative stimulation improves synaptic plasticity and functional outcomes after cerebral ischemia', Neural Regeneration Research, 14(11), pp. 1968-1976. doi: 10.4103/1673-5374.259618.

Huang, Y.-Z. et al. (2017) 'Plasticity induced by non-invasive transcranial brain stimulation: A position paper', Clinical Neurophysiology, 128(11), pp. 2318-2329. doi: 10.1016/j.clinph.2017.09.007.

Huang, Y. Z. et al. (2007) 'The after-effect of human theta burst stimulation is NMDA receptor dependent', Clinical Neurophysiology, 118(5), pp. 1028-1032. doi: 10.1016/j.clinph.2007.01.021.

Huang, Y. Z. (2016) 'What do we learn from the influence of motor activities on the after-effect of non-invasive brain stimulation?', Clinical Neurophysiology. International Federation of Clinical Neurophysiology, 127(2), pp. 1011-1012. doi: 10.1016/j.clinph.2015.11.015.

Huang, Z. L. et al. (2005) 'Adenosine A2A, but not A1, receptors mediate the arousal effect of caffeine', Nature Neuroscience, 8(7), pp. 858-859. doi: 10.1038/nn1491.

Huber, R. et al. (2008) 'Measures of cortical plasticity after transcranial paired associative stimulation predict changes in electroencephalogram slow-wave activity during subsequent sleep', Journal of Neuroscience, 28(31), pp. 7911-7918. doi: 10.1523/JNEUROSCI.163608.2008. 
Islam, N. et al. (1994) 'Anodal polarization induces protein kinase $\mathrm{C} \gamma(\mathrm{PKC} \gamma)$-like immunoreactivity in the rat cerebral cortex', Neuroscience Research, 21(2), pp. 169-172. doi: 10.1016/0168-0102(94)90159-7.

Islam, N. et al. (1995) 'Increase in the calcium level following anodal polarization in the rat brain', Brain Research, 684(2), pp. 206-208. doi: 10.1016/0006-8993(95)00434-R.

Isokawa, M. (2016) 'Caffeine-Induced Suppression of GABAergic Inhibition and CalciumIndependent Metaplasticity’, Neural Plasticity, 2016, pp. 1-7. doi: 10.1155/2016/1239629.

Jung, P. and Ziemann, U. (2009) 'Homeostatic and nonhomeostatic modulation of learning in human motor cortex', Journal of Neuroscience, 29(17), pp. 5597-5604. doi: 10.1523/JNEUROSCI.0222-09.2009.

Kamke, M. R. et al. (2012) 'Visual attentional load influences plasticity in the human motor cortex', Journal of Neuroscience, 32(20), pp. 7001-7008. doi: 10.1523/JNEUROSCI.1028-12.2012.

Karabanov, A. et al. (2015) 'Consensus Paper: Probing Homeostatic Plasticity of Human Cortex With Non-invasive Transcranial Brain Stimulation', Brain Stimulation, 8(3), pp. 442-454. doi: 10.1016/j.brs.2015.01.404.

Karabanov, A., Thielscher, A. and Siebner, H. R. (2016) 'Transcranial brain stimulation: Closing the loop between brain and stimulation', Current Opinion in Neurology, 29(4), pp. 397404. doi: 10.1097/WCO.0000000000000342.

Kasten, F. H., Dowsett, J. and Herrmann, C. S. (2016) 'Sustained aftereffect of $\alpha$-tACS lasts up to 70 min after stimulation', Frontiers in Human Neuroscience, 10(245), pp. 1-9. doi: 10.3389/fnhum.2016.00245.

Kasten, F. H. and Herrmann, C. S. (2017) 'Transcranial Alternating Current Stimulation (tACS) Enhances Mental Rotation Performance during and after Stimulation', Frontiers in Human Neuroscience, 11(2), pp. 1-16. doi: 10.3389/fnhum.2017.00002.

Kerkhofs, A. et al. (2018) 'Caffeine controls glutamatergic synaptic transmission and pyramidal neuron excitability in human neocortex', Frontiers in Pharmacology, 8(899), pp. 1-11. doi: 10.3389/fphar.2017.00899.

Kirschbaum, C. et al. (1999) 'Impact of gender, menstrual cycle phase, and oral contraceptives on the activity of the hypothalamus-pituitary-adrenal axis', Psychosomatic Medicine, 61(2), pp. 154-162. doi: 10.1097/00006842-199903000-00006.

Kirschfeld, K. (2005) 'The physical basis of alpha waves in the electroencephalogram and the origin of the "Berger effect", Biological Cybernetics, 92(3), pp. 177-185. doi: 10.1007/s00422-005-0547-1. 
Klomjai, W., Katz, R. and Lackmy-Vallée, A. (2015) 'Basic principles of transcranial magnetic stimulation (TMS) and repetitive TMS (rTMS)', Annals of Physical and Rehabilitation Medicine, pp. 208-213. doi: 10.1016/j.rehab.2015.05.005.

Kortuem, V. et al. (2019) 'Efficacy of tRNS and $140 \mathrm{~Hz}$ tACS on motor cortex excitability seemingly dependent on sensitivity to sham stimulation', Experimental Brain Research, 237(11), pp. 2885-2895. doi: 10.1007/s00221-019-05640-w.

Kraemer, S. et al. (2000) 'Time-of-day variations of indicators of attention: Performance, physiologic parameters, and self-assessment of sleepiness', Biological Psychiatry, 48(11), pp. 1069-1080. doi: 10.1016/S0006-3223(00)00908-2.

Kuhn, M. et al. (2016) 'Sleep recalibrates homeostatic and associative synaptic plasticity in the human cortex', Nature Communications, 7(1), p. 12455. doi: 10.1038/ncomms12455.

Kuhn, Y. A. et al. (2017) 'Adopting an external focus of attention alters intracortical inhibition within the primary motor cortex', Acta Physiologica, 220(2), pp. 289-299. doi: 10.1111/apha.12807.

Kuo, H. I. et al. (2013) 'Comparing cortical plasticity induced by conventional and high-definition $4 \times 1$ ring tDCS: A neurophysiological study’, Brain Stimulation, 6(4), pp. 644-648. doi: 10.1016/j.brs.2012.09.010.

Landolt, H. P. (2008) 'Sleep homeostasis: A role for adenosine in humans?', Biochemical Pharmacology, 75(11), pp. 2070-2079. doi: 10.1016/j.bcp.2008.02.024.

Lang, N. et al. (2011) 'Circadian modulation of GABA-mediated cortical inhibition', Cerebral Cortex, 21(10), pp. 2299-2306. doi: 10.1093/cercor/bhr003.

Lattari, E. et al. (2019) 'Effects of Transcranial Direct Current Stimulation With Caffeine Intake on Muscular Strength and Perceived Exertion', Journal of strength and conditioning research, 33(5), pp. 1237-1243. doi: 10.1519/JSC.0000000000003123.

Lazarski, J. P., Ridding, M. C. and Miles, T. S. (2002) 'Dexterity is not affected by fatigue-induced depression of human motor cortex excitability', Neuroscience Letters, 321(1-2), pp. 6972. doi: 10.1016/S0304-3940(02)00052-6.

Lee, S. et al. (2018) 'The influence of endogenous estrogen on transcranial direct current stimulation: A preliminary study', European Journal of Neuroscience, 48(4), pp. 20012012. doi: 10.1111/ejn.14085.

Leon-Sarmiento, F. E., Bara-Jimenez, W. and Wassermann, E. M. (2005) 'Visual deprivation effects on human motor cortex excitability', Neuroscience Letters, 389(1), pp. 17-20. doi: 10.1016/j.neulet.2005.06.061.

León, D. et al. (2002) 'Adenosine A1 receptor down-regulation in mothers and fetal brain after caffeine and theophylline treatments to pregnant rats', Journal of Neurochemistry, 82(3), pp. 625-634. doi: 10.1046/j.1471-4159.2002.01008.x. 
Liebetanz, D. et al. (2002) 'Pharmacological approach to the mechanisms of transcranial DCstimulation-induced after-effects of human motor cortex excitability', Brain, 125(10), pp. 2238-2247. doi: 10.1093/brain/awf238.

Liguori, A., Hughes, J. R. and Grass, J. A. (1997) 'Absorption and subjective effects of caffeine from coffee, cola and capsules', Pharmacology Biochemistry and Behavior, 58(3), pp. 721-726. doi: 10.1016/S0091-3057(97)00003-8.

Lisman, J. E. (2001) 'Three Ca2+ levels affect plasticity differently: The LTP zone, the LTD zone and no man's land', Journal of Physiology, 532(2), p. 285. doi: 10.1111/j.14697793.2001.0285f.x.

Lisman, J. and Spruston, N. (2010) 'Questions about STDP as a general model of synaptic plasticity', Frontiers in Synaptic Neuroscience, 2, pp. 1-5. doi: 10.3389/fnsyn.2010.00140.

López-Alonso, V. et al. (2014) 'Inter-individual variability in response to non-invasive brain stimulation paradigms', Brain Stimulation. Elsevier Ltd, 7(3), pp. 372-380. doi: 10.1016/j.brs.2014.02.004.

Lovallo, W. R. et al. (1996) 'Stress-like adrenocorticotropin responses to caffeine in young healthy men', Pharmacology Biochemistry and Behavior, 55(3), pp. 365-369. doi: 10.1016/S00913057(96)00105-0.

Lovallo, W. R. et al. (2006) 'Cortisol responses to mental stress, exercise, and meals following caffeine intake in men and women', Pharmacology Biochemistry and Behavior, 83(3), pp. 441-447. doi: 10.1016/j.pbb.2006.03.005.

Lüdtke, H. et al. (1998) 'Mathematical procedures in data recording and processing of pupillary fatigue waves', Vision Research, 38(19), pp. 2889-2896. doi: 10.1016/S00426989(98)00081-9.

Mäki, H. and Ilmoniemi, R. J. (2010) 'EEG oscillations and magnetically evoked motor potentials reflect motor system excitability in overlapping neuronal populations', Clinical Neurophysiology, 121(4), pp. 492-501. doi: 10.1016/j.clinph.2009.11.078.

Markram, H. (2011) 'A history of spike-timing-dependent plasticity', Frontiers in Synaptic Neuroscience, 3, pp. 1-24. doi: 10.3389/fnsyn.2011.00004.

Marquez-Ruiz, J. et al. (2012) 'Transcranial direct-current stimulation modulates synaptic mechanisms involved in associative learning in behaving rabbits', Proceedings of the National Academy of Sciences, 109(17), pp. 6710-6715. doi: 10.1073/pnas.1121147109.

Mateos-Aparicio, P. and Rodríguez-Moreno, A. (2019) 'The Impact of Studying Brain Plasticity', Frontiers in Cellular Neuroscience, 13(66), pp. 1-5. doi: 10.3389/fncel.2019.00066.

Matthaei, J. et al. (2016) 'Heritability of Caffeine Metabolism: Environmental Effects Masking Genetic Effects on CYP1A2 Activity but Not on NAT2', Clinical Pharmacology and Therapeutics, 100(6), pp. 606-616. doi: 10.1002/cpt.444. 
Mejia, E. G. de and Ramirez-Mares, M. V. (2014) 'Impact of caffeine and coffee on our health', Trends in Endocrinology \& Metabolism, 25(10), pp. 489-492. doi: 10.1016/j.tem.2014.07.003.

Merton, P. A. and Morton, H. B. (1980) 'Stimulation of the cerebral cortex in the intact human subject', Nature, 285(5762), p. 227. doi: 10.1038/285227a0.

Mesquita, R. N. O. et al. (2020) 'Effects of caffeine on neuromuscular function in a non-fatigued state and during fatiguing exercise', Experimental Physiology, 105(4), pp. 690-706. doi: 10.1113/EP088265.

Mezzullo, M. et al. (2016) 'Validation of an LC-MS/MS salivary assay for glucocorticoid status assessment: Evaluation of the diurnal fluctuation of cortisol and cortisone and of their association within and between serum and saliva', Journal of Steroid Biochemistry and Molecular Biology. Elsevier Ltd, 163(2015), pp. 103-112. doi: 10.1016/j.jsbmb.2016.04.012.

Milani, P. et al. (2010) 'Cortisol-induced effects on human cortical excitability', Brain Stimulation. Elsevier Inc, 3(3), pp. 131-139. doi: 10.1016/j.brs.2009.07.004.

Minkova, L. et al. (2019) 'Determinants of Inter-Individual Variability in Corticomotor Excitability Induced by Paired Associative Stimulation', Frontiers in Neuroscience, 13, pp. 1-14. doi: 10.3389/fnins.2019.00841.

Mitchell, D. C. et al. (2014) 'Beverage caffeine intakes in the U.S.', Food and Chemical Toxicology, 63, pp. 136-142. doi: 10.1016/j.fct.2013.10.042.

Moliadze, V. et al. (2012) 'Close to threshold transcranial electrical stimulation preferentially activates inhibitory networks before switching to excitation with higher intensities', Brain Stimulation, 5(4), pp. 505-511. doi: 10.1016/j.brs.2011.11.004.

Moliadze, V., Antal, A. and Paulus, W. (2010) 'Boosting brain excitability by transcranial high frequency stimulation in the ripple range', The Journal of Physiology, 588(24), pp. 48914904. doi: 10.1113/jphysiol.2010.196998.

Moreno-Duarte, I. et al. (2014) 'Chapter 2 - Transcranial Electrical Stimulation: Transcranial Direct Current Stimulation (tDCS), Transcranial Alternating Current Stimulation (tACS), Transcranial Pulsed Current Stimulation (tPCS), and Transcranial Random Noise Stimulation (tRNS)', in Cohen Kadosh, R. B. T.-T. S. B. (ed.) The Stimulated Brain: Cognitive Enhancement Using Non-Invasive Brain Stimulation. San Diego: Academic Press, pp. 35-59. doi: https://doi.org/10.1016/B978-0-12-404704-4.00002-8.

Mori, F. et al. (2011) 'Genetic variants of the NMDA receptor influence cortical excitability and plasticity in humans', Journal of Neurophysiology, 106(4), pp. 1637-1643. doi: 10.1152/jn.00318.2011. 
Müller-Dahlhaus, F. et al. (2015) 'Augmenting LTP-Like Plasticity in Human Motor Cortex by Spaced Paired Associative Stimulation', PLOS ONE. Edited by G. Foffani, 10(6), p. e0131020. doi: 10.1371/journal.pone.0131020.

Müller-Dahlhaus, F., Ziemann, U. and Classen, J. (2010) 'Plasticity resembling spike-timing dependent synaptic plasticity: the evidence in human cortex', Frontiers in Synaptic Neuroscience, 2(34), pp. 1-11. doi: 10.3389/fnsyn.2010.00034.

Müller-Dahlhaus, J. F. M. et al. (2008) 'Interindividual variability and age-dependency of motor cortical plasticity induced by paired associative stimulation', Experimental Brain Research, 187(3), pp. 467-475. doi: 10.1007/s00221-008-1319-7.

Nardone, R. et al. (2004) 'Changes in motor cortical excitability in humans following orally administered theophylline', Neuroscience Letters, 355(1-2), pp. 65-68. doi: 10.1016/j.neulet.2003.10.055.

Naro, A. et al. (2014) 'A Local Signature of LTP-Like Plasticity Induced by Repetitive Paired Associative Stimulation', Brain Topography, 28(2), pp. 238-249. doi: 10.1007/s10548014-0396-0.

Neuling, T., Rach, S. and Herrmann, C. S. (2013) 'Orchestrating neuronal networks: sustained after-effects of transcranial alternating current stimulation depend upon brain states', Frontiers in Human Neuroscience, 7, pp. 1-12. doi: 10.3389/fnhum.2013.00161.

Nguyen, B. N. et al. (2018) 'Acute caffeine ingestion affects surround suppression of perceived contrast', Journal of Psychopharmacology, 32(1), pp. 81-88. doi: $10.1177 / 0269881117725684$.

Nitsche, M. A. et al. (2004) 'Consolidation of human motor cortical neuroplasticity by Dcycloserine', Neuropsychopharmacology, 29(8), pp. 1573-1578. doi: 10.1038/sj.npp.1300517.

Nitsche, M. A. et al. (2007) 'Timing-dependent modulation of associative plasticity by general network excitability in the human motor cortex', Journal of Neuroscience, 27(14), pp. 3807-3812. doi: 10.1523/JNEUROSCI.5348-06.2007.

Nitsche, M. A. et al. (2012) 'The pharmacology of neuroplasticity induced by non-invasive brain stimulation: building models for the clinical use of CNS active drugs', The Journal of Physiology, 590(19), pp. 4641-4662. doi: 10.1113/jphysiol.2012.232975.

Nitsche, M. A. and Paulus, W. (2000) 'Excitability changes induced in the human motor cortex by weak transcranial direct current stimulation', The Journal of Physiology, 527(3), pp. 633639. doi: 10.1111/j.1469-7793.2000.t01-1-00633.x.

Nitsche, M. A. and Paulus, W. (2011) 'Transcranial direct current stimulation - Update 2011', Restorative Neurology and Neuroscience, 29(6), pp. 463-492. doi: 10.3233/RNN-20110618. 
Noreika, V. et al. (2020) 'Alertness fluctuations when performing a task modulate cortical evoked responses to transcranial magnetic stimulation', Neurolmage. Elsevier Inc., 223, p. 117305. doi: 10.1016/j.neuroimage.2020.117305.

Ogata, K. et al. (2019) 'Prestimulus cortical EEG oscillations can predict the excitability of the primary motor cortex', Brain Stimulation. Elsevier Ltd, 12(6), pp. 1508-1516. doi: 10.1016/j.brs.2019.06.013.

Oldfield, R. C. (1971) 'The assessment and analysis of handedness: The Edinburgh inventory', Neuropsychologia, 9(1), pp. 97-113. doi: 10.1016/0028-3932(71)90067-4.

Orth, M. et al. (2005) 'Caffeine has no effect on measures of cortical excitability', Clinical Neurophysiology, 116(2), pp. 308-314. doi: 10.1016/j.clinph.2004.08.012.

Oster, H. et al. (2017) 'The functional and clinical significance of the 24-hour rhythm of circulating glucocorticoids', Endocrine Reviews, 38(1), pp. 3-45. doi: 10.1210/er.2015-1080.

Ozdemir, R. A. et al. (2020) 'Individualized perturbation of the human connectome reveals reproducible biomarkers of network dynamics relevant to cognition', Proceedings of the National Academy of Sciences, 117(14), pp. 8115-8125. doi: 10.1073/pnas.1911240117.

Patz, M. D. et al. (2006) 'Modulation of the hypothalamo-pituitary-adrenocortical axis by caffeine', Psychoneuroendocrinology, 31(4), pp. 493-500. doi: 10.1016/j.psyneuen.2005.11.008.

Paulus, W. et al. (2008) 'State of the art: Pharmacologic effects on cortical excitability measures tested by transcranial magnetic stimulation', Brain Stimulation, 1(3), pp. 151-163. doi: 10.1016/j.brs.2008.06.002.

Paulus, W. (2011) 'Transcranial electrical stimulation (tES - tDCS; tRNS, tACS) methods', Neuropsychological Rehabilitation, 21(5), pp. 602-617. doi: 10.1080/09602011.2011.557292.

Paulus, W., Peterchev, A. V. and Ridding, M. (2013) 'Transcranial electric and magnetic stimulation: Technique and paradigms', in Handbook of Clinical Neurology, pp. 329-342. doi: 10.1016/B978-0-444-53497-2.00027-9.

Paulus, W. and Rothwell, J. C. (2016) 'Membrane resistance and shunting inhibition: Where biophysics meets state-dependent human neurophysiology', Journal of Physiology. Blackwell Publishing Ltd, 594(10), pp. 2719-2728. doi: 10.1113/JP271452.

Pavlides, C., Watanabe, Y. and McEwen, B. S. (1993) 'Effects of glucocorticoids on hippocampal long-term potentiation', Hippocampus, 3(2), pp. 183-192. doi: 10.1002/hipo.450030210.

Perogamvros, I. et al. (2010) 'Salivary cortisone is a potential biomarker for serum free cortisol', Journal of Clinical Endocrinology and Metabolism, 95(11), pp. 4951-4958. doi: 10.1210/jc.2010-1215. 
Pitskel, N. B. et al. (2007) 'Time-dependent changes in cortical excitability after prolonged visual deprivation', NeuroReport, 18(16), pp. 1703-1707. doi: 10.1097/WNR.0b013e3282f0d2c1.

Ploner, M. et al. (2006) 'Oscillatory activity reflects the excitability of the human somatosensory system', NeuroImage, 32(3), pp. 1231-1236. doi: 10.1016/j.neuroimage.2006.06.004.

Polanía, R. et al. (2012) 'The importance of timing in segregated theta phase-coupling for cognitive performance', Current Biology, 22(14), pp. 1314-1318. doi: 10.1016/j.cub.2012.05.021.

Polanía, R., Nitsche, M. A. and Ruff, C. C. (2018) 'Studying and modifying brain function with non-invasive brain stimulation', Nature Neuroscience, 21(2), pp. 174-187. doi: 10.1038/s41593-017-0054-4.

Pötter-Nerger, M. et al. (2009) 'Inducing homeostatic-like plasticity in human motor cortex through converging corticocortical inputs', Journal of Neurophysiology, 102(6), pp. 31803190. doi: 10.1152/jn.91046.2008.

de Quervain, D. J.-F. et al. (2000) 'Acute cortisone administration impairs retrieval of long-term declarative memory in humans', Nature Neuroscience, 3(4), pp. 313-314. doi: $10.1038 / 73873$.

Rahman, A. et al. (2013) 'Cellular effects of acute direct current stimulation: somatic and synaptic terminal effects', The Journal of Physiology, 591(10), pp. 2563-2578. doi: 10.1113/jphysiol.2012.247171.

Rahman, A., Lafon, B. and Bikson, M. (2015) Multilevel computational models for predicting the cellular effects of noninvasive brain stimulation. 1st edn, Progress in Brain Research. 1st edn. Elsevier B.V. doi: 10.1016/bs.pbr.2015.09.003.

Rawji, V. et al. (2018) 'tDCS changes in motor excitability are specific to orientation of current flow', Brain Stimulation. Elsevier Inc., 11(2), pp. 289-298. doi: 10.1016/j.brs.2017.11.001.

Reato, D. et al. (2013) 'Effects of weak transcranial alternating current stimulation on brain activity-a review of known mechanisms from animal studies', Frontiers in Human Neuroscience, 7(OCT), pp. 1-8. doi: 10.3389/fnhum.2013.00687.

Regen, F., Dorn, H. and Danker-Hopfe, H. (2013) 'Association between pupillary unrest index and waking electroencephalogram activity in sleep-deprived healthy adults', Sleep Medicine. Elsevier B.V., 14(9), pp. 902-912. doi: 10.1016/j.sleep.2013.02.003.

Reis, J. et al. (2006) 'Modulation of human motor cortex excitability by single doses of amantadine', Neuropsychopharmacology, 31(12), pp. 2758-2766. doi: 10.1038/sj.npp.1301122. 
Ridding, M. C. and Rothwell, J. C. (2007) 'Is there a future for therapeutic use of transcranial magnetic stimulation?', Nature Reviews Neuroscience, 8(7), pp. 559-567. doi: 10.1038/nrn2169.

Ridding, M. C. and Ziemann, U. (2010) 'Determinants of the induction of cortical plasticity by non-invasive brain stimulation in healthy subjects.', The Journal of physiology, 588(13), pp. 2291-304. doi: 10.1113/jphysiol.2010.190314.

Rinne, P. et al. (2018) 'Motor dexterity and strength depend upon integrity of the attention-control system', Proceedings of the National Academy of Sciences of the United States of America, 115(3), pp. E536-E545. doi: 10.1073/pnas.1715617115.

Romei, V., Rihs, T., et al. (2008) 'Resting electroencephalogram alpha-power over posterior sites indexes baseline visual cortex excitability', NeuroReport, 19(2), pp. 203-208. doi: 10.1097/WNR.0b013e3282f454c4.

Romei, V., Brodbeck, V., et al. (2008) 'Spontaneous fluctuations in posterior $\alpha$-band EEG activity reflect variability in excitability of human visual areas', Cerebral Cortex, 18(9), pp. 20102018. doi: $10.1093 /$ cercor/bhm229.

Rosenkranz, K. and Rothwell, J. C. (2006) 'Spatial attention affects sensorimotor reorganisation in human motor cortex', Experimental Brain Research, 170(1), pp. 97-108. doi: 10.1007/s00221-005-0173-0.

Rossi, S. et al. (2009) 'Safety, ethical considerations, and application guidelines for the use of transcranial magnetic stimulation in clinical practice and research', Clinical Neurophysiology, pp. 2008-2039. doi: 10.1016/j.clinph.2009.08.016.

Rossini, P. M. et al. (2015) 'Non-invasive electrical and magnetic stimulation of the brain, spinal cord, roots and peripheral nerves: Basic principles and procedures for routine clinical and research application: An updated report from an I.F.C.N. Committee', Clinical Neurophysiology. International Federation of Clinical Neurophysiology, 126(6), pp. 10711107. doi: 10.1016/j.clinph.2015.02.001.

Sachse, C. et al. (1999) 'Functional significance of a C $\rightarrow$ A polymorphism in intron I of the cytochrome P450 CYP1A2 gene tested with caffeine', British Journal of Clinical Pharmacology, 47(4), pp. 445-449. doi: 10.1046/j.1365-2125.1999.00898.x.

Saghazadeh, A., Esfahani, S. A. and Rezaei, N. (2016) 'Genetic polymorphisms and the adequacy of brain stimulation: state of the art', Expert Review of Neurotherapeutics, 16(9), pp. 10431054. doi: 10.1080/14737175.2016.1194202.

Sale, M. V., Ridding, M. C. and Nordstrom, M. A. (2008) 'Cortisol inhibits neuroplasticity induction in human motor cortex', Journal of Neuroscience, 28(33), pp. 8285-8293. doi: 10.1523/JNEUROSCI.1963-08.2008.

Sale, M. V, Ridding, M. C. and Nordstrom, M. A. (2007) 'Factors influencing the magnitude and reproducibility of corticomotor excitability changes induced by paired associative 
stimulation', Experimental Brain Research, 181(4), pp. 615-626. doi: 10.1007/s00221007-0960-x.

Sauseng, P. et al. (2009) 'Spontaneous locally restricted EEG alpha activity determines cortical excitability in the motor cortex', Neuropsychologia, 47(1), pp. 284-288. doi: 10.1016/j.neuropsychologia.2008.07.021.

Schulz, H. et al. (2014) 'Now i am ready - Now i am not: The influence of pre-TMS oscillations and corticomuscular coherence on motor-evoked potentials', Cerebral Cortex, 24(7), pp. 1708-1719. doi: 10.1093/cercor/bht024.

Schutter, D. J. L. G. and Hortensius, R. (2011) 'Brain oscillations and frequency-dependent modulation of cortical excitability’, Brain Stimulation. Elsevier Inc, 4(2), pp. 97-103. doi: 10.1016/j.brs.2010.07.002.

Scott, N. R., Chakraborty, J. and Marks, V. (1984) 'Determination of Caffeine, Theophylline and Theobromine in Serum and Saliva Using High-Performance Liquid Chromatography', Annals of Clinical Biochemistry, 21(2), pp. 120-124. doi: 10.1177/000456328402100208.

Shirota, Y. et al. (2017) 'Influence of concurrent finger movements on transcranial direct current stimulation (tDCS)-induced aftereffects', Frontiers in Behavioral Neuroscience. Frontiers Media S.A., 11(169), pp. 1-7. doi: 10.3389/fnbeh.2017.00169.

Silvanto, J., Muggleton, N. and Walsh, V. (2008) 'State-dependency in brain stimulation studies of perception and cognition', Trends in Cognitive Sciences, 12(12), pp. 447-454. doi: 10.1016/j.tics.2008.09.004.

Silverstein, J. et al. (2019) 'Paired Associative Stimulation as a Tool to Assess Plasticity Enhancers in Chronic Stroke', Frontiers in Neuroscience, 13(JUL), pp. 1-10. doi: 10.3389/fnins.2019.00792.

Sliva, D. D. et al. (2018) 'A prospective study of the impact of transcranial alternating current stimulation on EEG correlates of somatosensory perception', Frontiers in Psychology, 9(2117), pp. 1-17. doi: 10.3389/fpsyg.2018.02117.

Smith, M. . et al. (1999) 'Menstrual cycle effects on cotical excitability', Neurology, 53, pp. 20692072. doi: 10.1212/wn1.53.9.2064.

Solianik, R. et al. (2018) 'Task-relevant cognitive and motor functions are prioritized during prolonged speed-accuracy motor task performance', Experimental Brain Research, 236(6), pp. 1665-1678. doi: 10.1007/s00221-018-5251-1.

Stagg, C. J. et al. (2009) 'Polarity-sensitive modulation of cortical neurotransmitters by transcranial stimulation', Journal of Neuroscience, 29(16), pp. 5202-5206. doi: 10.1523/JNEUROSCI.4432-08.2009.

Stagg, C. J., Antal, A. and Nitsche, M. A. (2018) 'Physiology of Transcranial Direct Current Stimulation', Journal of ECT, 34(3), pp. 144-152. doi: 10.1097/YCT.0000000000000510. 
Stecher, H. I. et al. (2017) 'Ten Minutes of $\alpha$-tACS and Ambient Illumination Independently Modulate EEG $\alpha$-Power', Frontiers in Human Neuroscience, 11(May), pp. 1-10. doi: 10.3389/fnhum.2017.00257.

Stecher, H. I. and Herrmann, C. S. (2018) 'Absence of alpha-tACS aftereffects in darkness reveals importance of taking derivations of stimulation frequency and individual alpha variability into account', Frontiers in Psychology, 9(JUN). doi: 10.3389/fpsyg.2018.00984.

Stefan, K. et al. (2000) 'Induction of plasticity in the human motor cortex by paired associative stimulation', Brain, 123(3), pp. 572-584. doi: 10.1093/brain/123.3.572.

Stefan, K. et al. (2002) 'Mechanisms of enhancement of human motor cortex excitability induced by interventional paired associative stimulation', Journal of Physiology, 543(2), pp. 699708. doi: 10.1113/jphysiol.2002.023317.

Stefan, K., Wycislo, M. and Classen, J. (2004) 'Modulation of associative human motor cortical plasticity by attention', Journal of Neurophysiology, 92(1), pp. 66-72. doi: 10.1152/jn.00383.2003.

Strigaro, G. et al. (2015) 'Interaction between visual and motor cortex: A transcranial magnetic stimulation study', Journal of Physiology, 593(10), pp. 2365-2377. doi: 10.1113/JP270135.

Strüber, D. et al. (2015) 'On the possible role of stimulation duration for after-effects of transcranial alternating current stimulation', Frontiers in Cellular Neuroscience, 9(August), pp. 1-7. doi: 10.3389/fncel.2015.00311.

Suppa, A. et al. (2017) 'The associative brain at work: Evidence from paired associative stimulation studies in humans', Clinical Neurophysiology. International Federation of Clinical Neurophysiology, 128(11), pp. 2140-2164. doi: 10.1016/j.clinph.2017.08.003.

Talelli, P. et al. (2007) 'Pattern-specific role of the current orientation used to deliver Theta Burst Stimulation', Clinical Neurophysiology, 118(8), pp. 1815-1823. doi: 10.1016/j.clinph.2007.05.062.

Tavakoli, A. V. and Yun, K. (2017) 'Transcranial Alternating Current Stimulation (tACS) Mechanisms and Protocols', Frontiers in Cellular Neuroscience, 11(September), pp. 1-10. doi: 10.3389/fncel.2017.00214.

Thirugnanasambandam, N. et al. (2011) 'Dose-dependent nonlinear effect of L-DOPA on paired associative stimulation-induced neuroplasticity in humans', Journal of Neuroscience, 31(14), pp. 5294-5299. doi: 10.1523/JNEUROSCI.6258-10.2011.

Thut, G. et al. (2017) 'Guiding transcranial brain stimulation by EEG/MEG to interact with ongoing brain activity and associated functions: A position paper', Clinical Neurophysiology, 128(5), pp. 843-857. doi: 10.1016/j.clinph.2017.01.003. 
Thut, G. and Pascual-Leone, A. (2010) 'A review of combined TMS-EEG studies to characterize lasting effects of repetitive TMS and assess their usefulness in cognitive and clinical neuroscience', Brain Topography, 22(4), pp. 219-232. doi: 10.1007/s10548-009-0115-4.

Tolmacheva, A., Mäkelä, J. P. and Shulga, A. (2019) 'Increasing the frequency of peripheral component in paired associative stimulation strengthens its efficacy', Scientific Reports, 9(1), pp. 1-7. doi: 10.1038/s41598-019-40474-0.

Tononi, G. and Cirelli, C. (2006) 'Sleep function and synaptic homeostasis', Sleep Medicine Reviews, 10(1), pp. 49-62. doi: 10.1016/j.smrv.2005.05.002.

Tremblay, S. et al. (2017) 'Modulation of iTBS after-effects via concurrent directional TDCS: A proof of principle study', Brain Stimulation. Elsevier Inc., 10(4), pp. 744-747. doi: 10.1016/j.brs.2017.03.009.

Vargas, C. D. et al. (2004) 'The influence of hand posture on corticospinal excitability during motor imagery: A transcranial magnetic stimulation study', Cerebral Cortex. Oxford University Press, 14(11), pp. 1200-1206. doi: 10.1093/cercor/bhh080.

Veniero, D. et al. (2017) 'Inconsistent effects of parietal $\alpha$-tACS on Pseudoneglect across two experiments: A failed internal replication', Frontiers in Psychology, 8, pp. 1-14. doi: 10.3389/fpsyg.2017.00952.

Vossen, A., Gross, J. and Thut, G. (2015) 'Alpha Power Increase After Transcranial Alternating Current Stimulation at Alpha Frequency ( $\alpha$-tACS) Reflects Plastic Changes Rather Than Entrainment.', Brain stimulation, 8(3), pp. 499-508. doi: 10.1016/j.brs.2014.12.004.

Vosskuhl, J., Huster, R. J. and Herrmann, C. S. (2016) 'BOLD signal effects of transcranial alternating current stimulation (tACS) in the alpha range: A concurrent tACS-fMRI study', NeuroImage, 140, pp. 118-125. doi: 10.1016/j.neuroimage.2015.10.003.

Wach, C. et al. (2013) 'The effect of $10 \mathrm{~Hz}$ transcranial alternating current stimulation (tACS) on corticomuscular coherence', Frontiers in Human Neuroscience, 7(511), pp. 1-10. doi: 10.3389/fnhum.2013.00511.

Wankerl, K. et al. (2010) 'L-Type Voltage-Gated Ca2+ Channels: A Single Molecular Switch for Long-Term Potentiation/Long-Term Depression-Like Plasticity and Activity-Dependent Metaplasticity in Humans', Journal of Neuroscience, 30(18), pp. 6197-6204. doi: 10.1523/jneurosci.4673-09.2010.

Webster, K. and Ro, T. (2020) 'Visual Modulation of Resting State $\alpha$ Oscillations', eneuro, 7(1), pp. 1-12. doi: 10.1523/ENEURO.0268-19.2019.

Weise, D. et al. (2013) 'Microcircuit mechanisms involved in paired associative stimulationinduced depression of corticospinal excitability', Journal of Physiology, 591(19), pp. 4903-4920. doi: 10.1113/jphysiol.2013.253989. 
Weise, D. et al. (2017) 'Differential regulation of human paired associative stimulation-induced and theta-burst stimulation-induced plasticity by L-type and T-type Ca2+channels', Cerebral Cortex, 27(8), pp. 4010-4021. doi: 10.1093/cercor/bhw212.

Wiethoff, S., Hamada, M. and Rothwell, J. C. (2014) 'Variability in response to transcranial direct current stimulation of the motor cortex.', Brain stimulation. Elsevier Ltd, 7(3), pp. 46875. doi: 10.1016/j.brs.2014.02.003.

Wilhelm, B. et al. (2001) 'Daytime variations in central nervous system activation measured by a pupillographic sleepiness test', Journal of Sleep Research, 10(1), pp. 1-7. doi: 10.1046/j.1365-2869.2001.00239.x.

Wischnewski, M. et al. (2019) 'NMDA Receptor-Mediated Motor Cortex Plasticity After $20 \mathrm{~Hz}$ Transcranial Alternating Current Stimulation', Cerebral Cortex, 29(7), pp. 2924-2931. doi: 10.1093/cercor/bhy160.

Wischnewski, M. and Schutter, D. J. L. G. (2017) 'After-effects of transcranial alternating current stimulation on evoked delta and theta power', Clinical Neurophysiology. International Federation of Clinical Neurophysiology, 128(11), pp. 2227-2232. doi: 10.1016/j.clinph.2017.08.029.

Wischnewski, M., Schutter, D. J. L. G. and Nitsche, M. A. (2019) 'Effects of beta-tACS on corticospinal excitability: A meta-analysis', Brain Stimulation. doi: 10.1016/j.brs.2019.07.023.

Wolf, O. T. (2009) 'Stress and memory in humans: Twelve years of progress?', Brain Research. Elsevier B.V., 1293, pp. 142-154. doi: 10.1016/j.brainres.2009.04.013.

Wolters, A. et al. (2003) 'A temporally asymmetric Hebbian rule governing plasticity in the human motor cortex', Journal of Neurophysiology, 89(5), pp. 2339-2345. doi: 10.1152/jn.00900.2002.

Wolters, A. et al. (2005) 'Timing-dependent plasticity in human primary somatosensory cortex', Journal of Physiology, 565(3), pp. 1039-1052. doi: 10.1113/jphysiol.2005.084954.

Yasuda, R., Sabatini, B. L. and Svoboda, K. (2003) 'Plasticity of calcium channels in dendritic spines', Nature Neuroscience, 6(9), pp. 948-955. doi: 10.1038/nn1112.

Zaehle, T., Rach, S. and Herrmann, C. S. (2010) 'Transcranial Alternating Current Stimulation Enhances Individual Alpha Activity in Human EEG', PLoS ONE, 5(11), pp. 1-7. doi: 10.1371/journal.pone.0013766.

Zarkowski, P. et al. (2006) 'EEG and the Variance of Motor Evoked Potential Amplitude', Clinical EEG and Neuroscience, 37(3), pp. 247-251. doi: 10.1177/155005940603700316.

Zazio, A. et al. (2019) 'Perceptual and Physiological Consequences of Dark Adaptation: A TMSEEG Study’, Brain Topography, 32(5), pp. 773-782. doi: 10.1007/s10548-019-00715-x. 
Ziemann, U. et al. (2015) 'TMS and drugs revisited 2014', Clinical Neurophysiology. International Federation of Clinical Neurophysiology, 126(10), pp. 1847-1868. doi: 10.1016/j.clinph.2014.08.028.

Ziemann, U. and Siebner, H. R. (2015) 'Inter-subject and intersession variability of plasticity induction by non-invasive brain stimulation: Boon or bane?', Brain Stimulation. Elsevier, 8(3), pp. 662-663. doi: 10.1016/j.brs.2015.01.409.

Zrenner, C. et al. (2016) 'Closed-Loop Neuroscience and Non-Invasive Brain Stimulation: A Tale of Two Loops', Frontiers in Cellular Neuroscience, 10(92), pp. 1-7. doi: 10.3389/fncel.2016.00092.

Zrenner, C. et al. (2018) 'Real-time EEG-defined excitability states determine efficacy of TMSinduced plasticity in human motor cortex', Brain Stimulation. Elsevier Ltd, 11(2), pp. 374389. doi: 10.1016/j.brs.2017.11.016.

Zulkifly, M. F. M., Merkohitaj, O. and Paulus, W. (2020) 'Transcranial alternating current stimulation induced excitatory aftereffects are abolished by decaffeinated espresso and reversed into inhibition by espresso with caffeine', Clinical Neurophysiology, 131(3), pp. 778-779. doi: 10.1016/j.clinph.2019.11.062. 
APPENDIX A 


\section{APPENDIX A.1 CURRICULUM VITAE}

\section{Personal Information}

Name

: Mohd Faizal Mohd Zulkifly

Nationality

: Malaysian

Mobile Phone No $\quad$ : +4917628111560

E-mail Address : :mfzl_mzly@yahoo.com

$\underline{\text { Education }}$

\begin{tabular}{|c|c|c|c|}
\hline Year & Degree & Discipline & University/Work Place \\
\hline $\begin{array}{l}2016 \text { - } \\
\text { present }\end{array}$ & $\begin{array}{l}\text { PhD } \\
\text { Dissertation: "Modulation } \\
\text { of plasticity aftereffects at } \\
\text { the sensorimotor cortex by } \\
\text { transcranial electrical and } \\
\text { magnetic stimulation" } \\
\text { Supervisor: Prof. Dr. Walter } \\
\text { Paulus }\end{array}$ & $\begin{array}{l}\text { Neuroscience } \\
\text { (Sensory and } \\
\text { Motor) }\end{array}$ & $\begin{array}{l}\text { Georg-August University } \\
\text { Göttingen, Germany }\end{array}$ \\
\hline $\begin{array}{l}2012- \\
2014\end{array}$ & $\begin{array}{l}\text { Master in Clinical } \\
\text { Psychology } \\
\text { Thesis: "The influence of } \\
\text { demographic, clinical, } \\
\text { psychological and } \\
\text { functional determinants on } \\
\text { post-stroke cognitive } \\
\text { impairment at day care } \\
\text { stroke center, Malaysia" } \\
\text { Supervisor: Dr. Shazli Ezzat } \\
\text { Ghazali }\end{array}$ & $\begin{array}{l}\text { Clinical } \\
\text { Psychology }\end{array}$ & $\begin{array}{l}\text { National University of } \\
\text { Malaysia (UKM), } \\
\text { Malaysia }\end{array}$ \\
\hline
\end{tabular}




\begin{tabular}{|c|c|c|c|}
\hline $\begin{array}{l}2011- \\
2012\end{array}$ & $\begin{array}{l}\text { Postgraduate Diploma in } \\
\text { Health Psychology } \\
\text { Thesis: "The influence of } \\
\text { recovery locus of control } \\
\text { (RLOC) and posttraumatic } \\
\text { stress symptom (PTSS) } \\
\text { towards physical } \\
\text { functioning of stroke } \\
\text { patients" }\end{array}$ & $\begin{array}{l}\text { Health } \\
\text { Psychology }\end{array}$ & $\begin{array}{l}\text { National University of } \\
\text { Malaysia (UKM), } \\
\text { Malaysia }\end{array}$ \\
\hline & $\begin{array}{l}\text { Supervisor: Dr. Shazli Ezzat } \\
\text { Ghazali }\end{array}$ & & \\
\hline $\begin{array}{l}2007- \\
2011\end{array}$ & $\begin{array}{l}\text { Bachelor of Biomedical } \\
\text { Sciences (Hons.) } \\
\text { Thesis: "Cytogenetic } \\
\text { analysis of exfoliated } \\
\text { buccal cells of farmers who } \\
\text { exposed to pesticides in } \\
\text { Bachok and Pasir Puteh, } \\
\text { Kelantan" } \\
\text { Supervisor: Assoc. Prof. Dr. } \\
\text { Zariyantey Abdul Hamid }\end{array}$ & $\begin{array}{l}\text { Biomedical } \\
\text { Sciences }\end{array}$ & $\begin{array}{l}\text { National University of } \\
\text { Malaysia (UKM), } \\
\text { Malaysia }\end{array}$ \\
\hline
\end{tabular}

\section{$\underline{\text { Field(s) of Specialization }}$}

- Clinical Psychology

- Neuroscience (Sensory and Motor)

- Neurophysiology

- Neuroimaging

\section{$\underline{\text { Research Area }}$}

- Brain plasticity

- Neuronal connectivity 
- Noninvasive brain stimulation (i.e. TMS, tES)

- Neurorehabilitation

$\underline{\text { Skills }}$

Programming

Document preparation

Operating system platform

Languages
Matlab

Microsoft Office

Windows, Linux (Ubuntu)

English (Fluent), Malay (Native)

\section{Publications}

[1] Zulkifly MFM, Merkohitaj O, Paulus W. Transcranial alternating current stimulation induced excitatory aftereffects are abolished by decaffeinated espresso and reversed into inhibition by espresso with caffeine. Clin Neurophysiol 2019. https://doi.org/10.1016/j.clinph.2019.11.062.

[2] Mohd Zulkifly MF, Ghazali SE, Che Din N, Desa A, Raymond AA. The ability of recovery locus of control scale (RLOC) and post-traumatic stress symptoms (PTSS) to predict the physical functioning of stroke patients. Malaysian J Med Sci 2015;22:31-41.

[3] Mohd Zulkifly MF, Ghazali SE, Che Din N, Subramaniam P. The influence of demographic, clinical, psychological and functional determinants on post-stroke cognitive impairment at day care stroke center, Malaysia. Malaysian J Med Sci 2016;23:53-64.

[4] Mohd Zulkifly MF, Ghazali SE, Che Din N, Singh DKA, Subramaniam P. A Review of Risk Factors for Cognitive Impairment in Stroke Survivors. Sci World J 2016;2016:1-16. https://doi.org/10.1155/2016/3456943.

[5] Abdul Hamid Z, Mohd Zulkifly MF, Hamid A, Lubis SH, Mohammad N, Ishak I, et al. The association of nuclear abnormalities in exfoliated buccal epithelial cells with the health status of different agricultural activities farmers in Peninsular Malaysia. Genes Environ 2016;38:7. https://doi.org/10.1186/s41021-016-0032-1.

[6] Shazli Ezzat Ghazali, Mohd Faizal Mohd Zulkifly, Normah Che Din, Asmawati Desa, Raymond Azman Ali. Hubungan antara faktor demografik dan klinikal dengan kefungsian fizikal pesakit strok. Malaysian Journal of Health Sciences 2016, 14(2): 1-15. 
$\underline{\text { Scholarships }}$

2016 - present

2012-2014

2007-2011
Fellowship in University of Science Malaysia (USM)

MyBrain15 Scholarship, Ministry of Higher Education, Malaysia (MOHE)

JPA Program Ijazah Dalam Negara (PIDN) Scholarship, Ministry of Education, Malaysia 